https://theses.gla.ac.uk/

Theses Digitisation:

https://www.gla.ac.uk/myglasgow/research/enlighten/theses/digitisation/

This is a digitised version of the original print thesis.

Copyright and moral rights for this work are retained by the author

A copy can be downloaded for personal non-commercial research or study, without prior permission or charge

This work cannot be reproduced or quoted extensively from without first obtaining permission in writing from the author

The content must not be changed in any way or sold commercially in any format or medium without the formal permission of the author

When referring to this work, full bibliographic details including the author, title, awarding institution and date of the thesis must be given

Enlighten: Theses

https://theses.gla.ac.uk/

research-enlighten@glasgow.ac.uk 


\title{
Polarisation Transfer in Proton Compton Scattering at High Momentum Transfer
}

\author{
by
}

David Jonathan Hamilton

Presented as a Thesis for the degree of Doctor of Philosophy

\author{
Nuclear Physics Group \\ Department of Physics and Astronomy \\ University of Glasgow
}

(C) D. J. Hamilton 2004 
ProQuest Number: 10753963

All rights reserved

INFORMATION TO ALL USERS

The quality of this reproduction is dependent upon the quality of the copy submitted.

In the unlikely event that the author did not send a complete manuscript and there are missing pages, these will be noted. Also, if material had to be removed, a note will indicate the deletion.

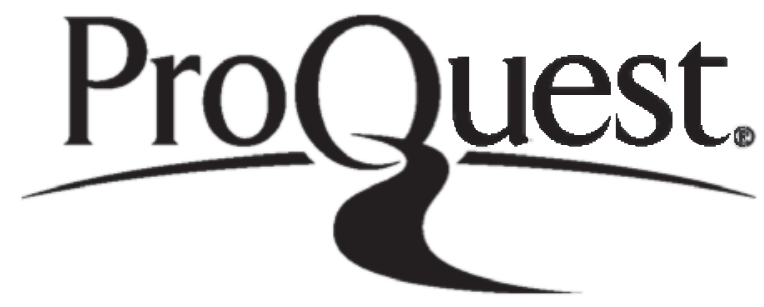

ProQuest 10753963

Published by ProQuest LLC (2018). Copyright of the Dissertation is held by the Author.

All rights reserved.

This work is protected against unauthorized copying under Title 17, United States Code Microform Edition (C) ProQuest LLC.

ProQuest LLC.

789 East Eisenhower Parkway

P.O. Box 1346

Ann Arbor, Ml $48106-1346$ 
GLASGOW

UNDERSITY LlisRARY: 


\begin{abstract}
The Jefferson Lab Hall A experiment E99-114 comprised a series of measurements to explore proton Compton scattering at high momentum transfer. For the first time, the polarisation transfer observables in the $p\left(\vec{\gamma}, \gamma^{\prime} \vec{p}\right)$ reaction were measured in the $\mathrm{GeV}$ energy range, where it is believed that quark-gluon degrees of freedom begin to dominate.

The experiment utilised a circularly polarised photon beam incident on a liquid hydrogen target, with the scattered photon and recoil proton detected in a lead-glass calorimeter and a magnetic spectrometer, respectively. A high efficiency proton polarimeter located at the spectrometer focal plane was used to extract the beam helicity asymmetry, from which the polarisation transfer components $\left(K_{L L}\right.$ and $\left.K_{L T}\right)$ at a centre-of-mass energy squared $s=6.9 \mathrm{GeV}^{2}$ and momentum transfer $t=-4.0 \mathrm{GeV}^{2}$ were determined. This analysis involved modelling the precession of the proton spin in the magnetic optics of the spectrometer, as well as calibration of the polarimeter analysing power via the $p\left(\vec{e}, e^{\prime} \vec{p}\right)$ reaction.

The real power of this polarisation measurement lies in the fact that the two reaction mechanisms assumed to contribute in this kinematic domain - the leading twist pQCD approach and the handbag factorisation - give drastically different predictions for $K_{L L}$ at wide angles. The results, $K_{L T}=0.111 \pm 0.078$ (stat) \pm 0.04 (syst),$K_{L L}=0.677 \pm 0.083$ (stat) \pm 0.04 (syst), indicate unambiguous agreement with the handbag mechanism and disagreement with the pQCD predictions. Furthermore, in terms of the non-perturbative structure of the proton the results highlight the importance of states with non-zero quark orbital angular momentum.
\end{abstract}




\section{Declaration}

The data presented in this thesis were obtained as part of the RCS collaboration at the U.S. Department of Energy's Jefferson Lab in Virginia and the University of Glasgow's Nuclear Physics Group. I participated fully in the preparation and execution of the experiment. The analysis of the experimental data is my own work. This thesis was composed by myself.

\section{David J. Hamilton}

July 2004 


\section{Contents}

1 Introduction $\quad 1$

2 Nucleon Structure $\quad 5$

2.1 Exclusive Structure: Form Factors . . . . . . . . . . . . 5

2.2 Inclusive Structure: Parton Distributions . . . . . . . . . . . 8

2.3 Generalised Parton Distributions . . . . . . . . . . . 11

3 Proton Compton Scattering $\quad 16$

3.1 Low Energy Regime . . . . . . . . . . . . . . . . 16

3.2 Asymptotically Large Energies: pQCD . . . . . . . . . . . . . . . 18

3.2.1 Leading Twist Factorisation . . . . . . . . . . . . . 19

3.2.2 Calculations for Compton Scattering . . . . . . . . 20

3.2.3 Predictions and Comparison with Available Data . . . . . 22

3.3 The Handbag Factorisation . . . . . . . . . . . . . 25

3.3.1 The Soft Overlap Mechanism . . . . . . . . . 26

3.3.2 The Modelling of Form Factors . . . . . . . . . . . 28

3.3 .3 Predictions .................... 31

3.4 Other Recent Calculations . . . . . . . . . . . . . 33

3.4.1 Relativistic Quark Models ... . . . . . . . . . 33

3.4.2 Regge Exchange Mechanism . . . . . . . . . . . 35

3.5 Summary ..................... 36

4 Experimental Apparatus $\quad 39$

4.1 The Thomas Jefferson National Accelerator Facility . . . . . . . . 40

4.2 Experimental Hall A . . . . . . . . . . . . . . . . 42

4.2.1 Beamline Apparatus .............. . . 42

4.2.2 Cryogenic Target System . . . . . . . . . . . . . . 44

4.2.3 High Resolution Spectrometer . . . . . . . . . . 46 
4.2.4 HRS Focal Plane Detectors . . . . . . . . . . . . . 48

4.3 Focal Plane Polarimeter . . . . . . . . . . . . . . . . . . 50

4.3.1 Recoil Proton Polarimetry . . . . . . . . . . . . 50

4.3 .2 Choice of Analyser . . . . . . . . . . . . . . 51

4.3.3 Straw Chambers ................ 52

4.4 RCS Photon Spectrometer . . . . . . . . . . . . . 53

4.4.1 Electromagnetic Showers . . . . . . . . . . . 54

4.4.2 Electromagnetic Calorimeter . . . . . . . . . . 55

4.4 .3 Electron Rejection . . . . . . . . . . . . 57

4.5 Data Acquisition . . . . . . . . . . . . . . 58

4.5.1 Trigger Scheme . . . . . . . . . . . . . . 59

4.5.2 The Data Readout Software . . . . . . . . . . . . 60

5 Data Analysis: Event Selection $\quad 63$

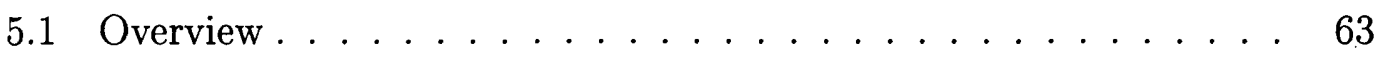

5.2 Hadron Analysis . . . . . . . . . . . . . . 64

5.2.1 VDC Track Reconstruction ............. 65

5.2.2 Target Coordinate Reconstruction . . . . . . . . 66

5.3 Photon Analysis . . . . . . . . . . . . . . . . . . . . 69

5.3.1 Calorimeter Shower Reconstruction . . . . . . . . . 69

5.3.2 Calorimeter Energy Calibration . . . . . . . . . 71

5.4 Production Data . . . . . . . . . . . . . 72

5.4.1 Event Identification . . . . . . . . . . . . 74

5.4.2 Background Correction ............ . 79

5.5 Calibration Data .................. 81

6 Data Analysis: Polarisation Observables $\quad 84$

6.1 Extraction of Focal Plane Asymmetries . . . . . . . . . . . . . 84

6.1.1 Straw Chamber Tracking . . . . . . . . . . . 85

6.1.2 Straw Chamber Alignment . . . . . . . . . . . 87

6.1.3 Calculation of Scattering Observables . . . . . . . . . 90

6.1.4 Azimuthal Asymmetries . . . . . . . . . . . . 93

6.2 Spin Transport . . . . . . . . . . . . . . . . 97

6.2.1 Simple Dipole Approximation . . . . . . . . . . 98

6.2.2 COSY Model . . . . . . . . . . . . . 99

6.2.3 Coordinate Systems at the Target . . . . . . . . . 101 
6.3 Maximum likelihood Determination of Polarisation Observables ... . . . . . . . . . . . 104

6.4 Analysing Power Calibration . . . . . . . . . . . . . 107

7 Results and Discussion $\quad 113$

7.1 Raw Polarisation Results . . . . . . . . . . . . . . . . . 113

7.1.1 Background Polarisation . . . . . . . . . . . . 114

7.1.2 Final Results for $K_{L T}$ and $K_{L L} \ldots \ldots \ldots \ldots$

7.2 Systematic Uncertainties . . . . . . . . . . . . . 118

7.2.1 $\pi^{0}$ Background Correction . . . . . . . . . 120

7.2.2 Total Systematic Uncertainty . . . . . . . . . . . . . . 122

7.3 Conclusions . . . . . . . . . . . . . . . . . . . 123

7.3.1 The Factorisation Scheme . . . . . . . . . . . . . . 124

7.3.2 Proton Structure within the Handbag Factorisation . . . . 125

7.3.3 Future Measurements . . . . . . . . . . . . 129

$\begin{array}{ll}\text { A Kinematic Calculations } & \mathbf{1 3 0}\end{array}$

B Beam Polarisation 135

$\begin{array}{ll}\text { Bibliography } & 138\end{array}$ 


\section{List of Figures}

2.1 Leading order diagrams for $p\left(e, e^{\prime} p\right)$ and $p\left(e, e^{\prime}\right) X \ldots \ldots \ldots$

2.2 The handbag diagram for hard exclusive processes . . . . . . . 11

2.3 Parameterisation of the GPD $H^{u}(x, \xi, t=0) \ldots \ldots \ldots 14$

3.1 Leading twist factorisation of exclusive Compton scattering . . . 20

3.2 pQCD differential cross section and $A_{L L}$ predictions . . . . . 22

3.3 Definition of the CM proton spin observables . . . . . . . . . . 24

3.4 The handbag factorisation of exclusive Compton scattering . . . 26

3.5 Three parameterisations of the form factors $R_{V}$ and $R_{A} \ldots \ldots \quad 30$

3.6 Soft overlap predictions for cross section and spin observables . . 32

3.7 LFCBM predictions for the longitudinal spin observables . . . . . 34

3.8 Regge exchange predictions for Compton spin observables . . . . 35

3.9 All of the currently available theoretical predictions for $K_{L L} \ldots \ldots 37$

4.1 Schematic of the experimental layout . . . . . . . . . . 40

4.2 The layout of the CEBAF machine at JLab . . . . . . . . . . 41

4.3 Electron and photon bremsstrahlung energy spectra . . . . . . 46

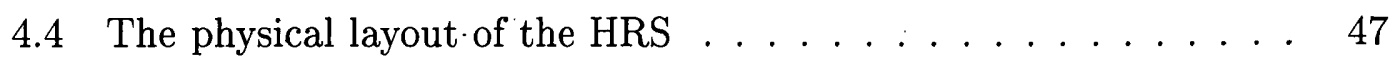

4.5 Diagram of the HRS-left detector package . . . . . . . . 48

4.6 Positioning of the Vertical Drift Chambers . . . . . . . . . 49

4.7 Dual analyser configuration of the Hall A FPP . . . . . . . . 52

4.8 Diagram of the RCS electromagnetic calorimeter . . . . . . 55

4.9 Measured field-map for the RCS deflection magnet . . . . . . . 58

4.10 A description of the production of the photon trigger $\ldots \ldots .60$

4.11 The coincidence trigger scheme for the RCS experiment . . . . . 61

5.1 Reconstructed sieve-slit position and proton trajectory . . . . . 68

5.2 Development of an electromagnetic shower in a lead-glass array . 70

5.3 Difference between measured and predicted electron energy . . . 72 
5.4 Timing, calorimeter energy and incident energy spectra . . . . . 75

5.5 Event indentifcation via the kinematic variables $\delta x$ and $\delta y \quad \ldots \quad 78$

5.6 The relative distributions of ep and potential $e p \gamma$ events . . . . 80

5.7 Fitting slices to the $\delta x$ vs $\delta y$ spectrum . . . . . . . . . . 81

5.8 Reconstructed kinematic variables for the calibration data . . . . 83

6.1 Straw chamber LE and (LE-TE) timing spectra . . . . . . . . 85

6.2 Difference between measured and reference trajectories . . . . . . 89

6.3 Definition of angles in the FPP coordinate system . . . . . . . . . 90

6.4 Distribtuion of polar scattering angle in both analysers . . . . . . 92

6.5 Spectra of the closest approach observables $s_{\text {close }}$ and $z_{\text {close }} \ldots$. . 94

6.6 Beam-helicity asymmetries for $\pi^{0}$ background events . . . . . . . 96

6.7 STM comparison between COSY and simple dipole models . . . . 99

6.8 Definition of proton polarisation in the ep scattering frame . . . 102

6.9 Sachs form factor ratio for the proton . . . . . . . . . . . . 108

6.10 Comparison of analysing powers with previous FPP data . . . . 110

6.11 Measured differential efficiency of both polarimeters . . . . . . . 111

7.1 Homogeniety of the background polarisation . . . . . . . . . . 115

7.2 Results and statistical error for different choices of $n_{\text {sigma }}$. . . . 117

7.3 Effect of varying the $n_{\text {sigma }}$ and $E_{\text {calo }}$ cuts . . . . . . . . . . . 121

7.4 Comparison between the final systematic uncertainties . . . . . . 123

7.5 Presentation of the result for $K_{L L} \ldots \ldots$. . . . . . . . . . . . 124

7.6 Comparsion between zero and non-zero helicity-flip amplitudes . . 127

7.7 Comparison between the hard subprocess and full calculations . . 127

A.1 The three coordinate systems used in kinematic calculations . . . 131

B.1 Stability of beam polarisation with time $\ldots \ldots \ldots 137$ 


\section{List of Tables}

4.1 Electron beam properties measured during the experiment . . . . 43

4.2 Spectrometer characteristics of the HRS-left . . . . . . . . 47

4.3 Active area covered by the four FPP straw chambers . . . . . 53

4.4 Physical properties of TF-1 lead-glass . . . . . . . . . 55

5.1 Production data kinematics and experimental conditions . . . 74

5.2 Table of calibration data kinematics . . . . . . . . . . . . 82

6.1 FPP false asymmetry coefficients $\ldots \ldots \ldots \ldots \ldots$

6.2 Average analysing power and FOM $\ldots \ldots \ldots \ldots$ 


\section{Chapter 1}

\section{Introduction}

Hadron physics is the study of the strongly interacting particles of nature. The most stable hadrons are those which make up the atomic nucleus, and in doing so account for almost all the mass in our solar system: the proton and neutron. In spite of the abundance of these particles the force governing their fundamental dynamics, the very force which binds them together and prevents the nucleus flying apart, is the least well understood of the four fundamental forces of nature. This is hardly surprising given the description of the strong interaction in terms of Quantum ChromoDynamics (QCD), wherein the nucleons are the lowest mass excitations of the labyrinthine amalgamation of quark and gluon condensates which form the QCD vacuum. All of the key features associated with the QCD Lagrangian are exhibited by the nucleon: colour confinement, asymptotic freedom and spontaneously broken chiral symmetry. A study of the nucleon, therefore, is a study of the strong interaction and QCD.

QCD is a renormalisable field theory based upon the principle of local gauge invariance under the exchange of colour. It describes the strong force in terms of fermion fields of a given colour charge, famously called quarks by Gell-Mann, interacting through the exchange of massless gauge bosons known as gluons. The hallmark of QCD which distinguishes it from the electroweak theory is the fact that these gluons possess non-zero colour charge (unlike the photons in electromagnetism which carry no electric charge). Chief amongst the resultant consequences is the property of colour confinement, whereby quarks and gluons are not permitted to exist as isolated free particles, but must combine to form colourneutral singlets: the three-quark baryons, of which the nucleons are the most stable, and the quark/anti-quark mesons. To date, the quantum numbers of all known strongly interacting particles have been accounted for using a model based 
upon the non-Abelian internal $S U(3)_{c}$ symmetry of QCD for elementary quarks of six different flavours (these are labeled in order of increasing mass as $\mathrm{u}, \mathrm{d}, \mathrm{s}$, $c, b$ and $t)$.

While the static properties of the nucleon can be described in this manner, the extreme non-linear nature of the strong force means that the dynamical properties of its constituents vary dramatically depending on the momentum scale at which it is probed. This leads to an unavoidable and profound dichotomy: large momentum behaviour of the nucleon can accurately be described by the quark and gluon fields of which it is composed, but this same description fails at low momentum where successful models have to rely instead on effective hadronic degrees of freedom. The problem is related to the asymptotic freedom of QCD, which is responsible for the fact that the force between the quarks within the nucleon becomes weaker as they move closer together.

For scattering processes involving the nucleon in the high momentum (hard) domain, perturbative QCD (pQCD) can be used to express the physical amplitudes as a perturbative series in the strong coupling constant $\left(\alpha_{s}\right)$ in a manner analogous to Quantum Electrodynamics (QED). However, renormalisation of the field theory is required for such a series to converge, and is achieved by the introduction of an arbitrary mass scale. This, in turn, leads to the running of the coupling strength of the strong interaction. That is to say, the effective strength is not a constant, but varies significantly depending on the momentum scale, decreasing logarithmically at large momentum transfers. This is why an increase in momentum not only implies a shorter distance scale but a decrease in the effective coupling, which causes the perturbative expansion to converge and allows for rigorous predictions based upon the quark and gluon fields alone.

Asymptotic freedom also means that the relatively large running coupling strength associated with the low momentum (soft) regime renders a perturbative expansion in $\alpha_{s}$ useless at large distance scales, such as typical hadronic sizes or larger. No rigorous solutions are available for non-perturbative QCD and nucleon behaviour at comparatively low energies, so effective theories become necessary. These are based upon identifying appropriate hadronic degrees of freedom for a particular momentum range and determining their characteristics. A variety of methods, each with their own limitations, have been employed over the years: QCD sum rules are formulated using the underlying symmetries of the strong interaction; many models are available based upon effective Lagrangians to approximate QCD at low energy; and more recently, computationally intensive 
calculations of physical quantities on a discretised Euclidean space-time lattice have been performed.

One feature common to all these approaches is the critical role played by the non-perturbative vacuum - a fact which inextricably links low energy QCD to the phenomenon of spontaneously broken chiral symmetry. If the light quarks are approximated as massless ( $u$ and $d$ quarks masses are believed to be around two to three orders of magnitude smaller than the mass of the nucleon), the conservation of their left and right-handedness introduces an additional layer of symmetry into any effective Lagrangian. This chiral symmetry is not exact and can be spontaneously (or dynamically) broken by the non-zero mass of confined quarks, which leads to these quarks developing large effective masses through interactions with the vacuum. The picture that one is left with, therefore, is of a nucleon consisting not only of three valence-quarks but a complex sea of quark/anti-quarks pairs and gluons, the characteristics of which are contingent on the momentum scale involved.

Experimentalists have been exploring the electromagnetic response of the nucleon for more than fifty years now. These measurements have been largely focused towards to the extreme upper and lower ends of the spectrum of momentum transferred to the nucleon. The intermediate region involving moderately large momentum transfer, where there is significant interplay between soft and hard physics and therefore between quark-gluon and meson-baryon degrees of freedom, has long since been identified as a fertile testing ground for models of the strong interaction. Precise experimental data in this regime, however, is relatively sparse due to the difficulties associated with taking such measurements, such as very low cross sections. The advent of large energy, high duty-factor electron accelerators, like the $6 \mathrm{GeV}$ machine at Jefferson Lab, has meant that a body of good quality data has become available in recent years to test and constrain the wealth of theoretical calculations in this field.

Real Compton scattering is a clean and potentially powerful probe of the nucleon, yet at moderately large momentum transfer it is one of the least used. The present measurement of polarisation observables for Compton scattering from the proton is the first of its kind in this regime. Complemented by a measurement of the differential cross section over a wide kinematic range - analysis of which is well underway - the data provides insight into both the non-perturbative structure of the proton and the underlying reaction mechanism for this process. Theoretical descriptions of the reaction mechanism are subtly linked to the manner in which 
the momentum transferred to the proton is shared between its constituents. Two schools of thought, which in some respects represent extreme cases, have emerged in recent years. The first of these is the PQCD approach, which is believed to dominate at very high energies and explicitly involves all three valence quarks of the proton, with momentum transferred between them via hard gluon exchange. The other approach involves the handbag mechanism, in which only one of the valence quarks is active in the scattering process and the momentum is transferred from this quark to the other constituents through overlaps of high momentum components of the proton wave function. Until fairly recently, the paradigm for describing hard exclusive processes such as proton Compton scattering has been the pQCD approach; it is likely to be superseded for moderately large energies, such as those presently accessible at Jefferson Lab, by the handbag mechanism. 


\section{Chapter 2}

\section{Nucleon Structure}

Since the 1930's and Stern's famous discovery that the nucleon's magnetic moment deviated strongly from the value expected for a structureless Dirac particle, the study of the structure of the nucleon has proven to be one of the most interesting and challenging fields in physics. Following those early days, the charge and current distributions within the nucleon have been accurately measured; irrefutable evidence for the existence of Feynman's partons - the point-like particles which make up the nucleon - has been uncovered; and a rigorous approach linking these partons to the more fundamental quarks and gluons of Quantum ChromoDynamics (QCD) has been developed. One of the latest, and in many ways the most elegant, approaches is the non-specific representation of nucleon structure in terms of Generalised Parton Distributions (GPD). It is within this framework that there has been intense recent interest in the family of hard exclusive reactions. In this section we provide a simple review of the progress made in understanding the nucleon, introducing the necessary formalism and key concepts along the way. An excellent overview of the field of nucleon structure and a fuller description than what follows can be found in ref. [1].

\subsection{Exclusive Structure: Form Factors}

A vast number of experiments have revealed the capacity of electromagnetic probes to precisely examine the structure of the nucleon. Since the electromagnetic interaction is so well understood, through the theory of Quantum ElectroDynamics (QED), the scattering of a lepton beam from a nucleon target, in a manner analogous to diffraction in optics, provides an accurate and decisive method of exploration. So much so, in fact, that many observers have likened 
this procedure to a microsurgery of the nucleon. The spatial resolution achievable in such an investigation depends only on the effective wavelength of the interacting virtual photon, and therefore, on the momentum transferred to the nucleon.

Interpretation of the experimental data is based upon the the paradigm of intrinsic nucleon structure introducing characteristic deviations from point-like behaviour, which leads to the following generalisations:

1. To parameterise the source of these deviations (i.e. the unknown aspects of the scattering object), a form factor (or structure function) is introduced in the matrix elements of the electromagnetic current between nucleon states.

2. Because of the clear relationship between momentum transfer and spatial resolution, the form factor of an object of finite size, and presumably therefore with internal structure, must depend on the momentum transferred in the reaction.

The archetypal electromagnetic investigation of the nucleon is elastic electronproton scattering: $e p \rightarrow e^{\prime} p$, measurements of which gave the first appraisal of the size of the proton in the mid-1950's [2]. The term elastic scattering is used to indicate that both the transferred energy $\nu$, and three-momentum $\mathbf{q}$, are absorbed by the recoiling proton as a whole (that is, without any intermediate excitation of the proton from its ground state). The leading order Feynman diagram for this process is shown in fig. 2.1(a).

Probing the nucleon at dimensions of $\sim 1 \mathrm{fm}\left(10^{-15} \mathrm{~m}\right)$ requires a virtual photon wavelength of the same order, and therefore highly relativistic electrons. If the proton were simply a structureless particle with Dirac magnetic moment, the case of relativistic electron scattering from such a particle, taking into account the electron spin and the fact that the proton will recoil in the process, is described by the familiar Mott cross section:

$$
\left(\frac{d \sigma}{d \Omega}\right)_{M o t t}=\frac{\alpha_{e m}^{2}}{4 E^{2} \sin ^{4} \theta / 2} \frac{E^{\prime}}{E} \cos ^{2} \frac{\theta}{2},
$$

where $\alpha_{e m}$ is the fine structure constant, and the initial and scattered electron energies, $E$ and $E^{\prime}$, are related (through two-body kinematics) to the scattering angle $\theta$. The relativistic nature of these calculations necessitates the use of the 

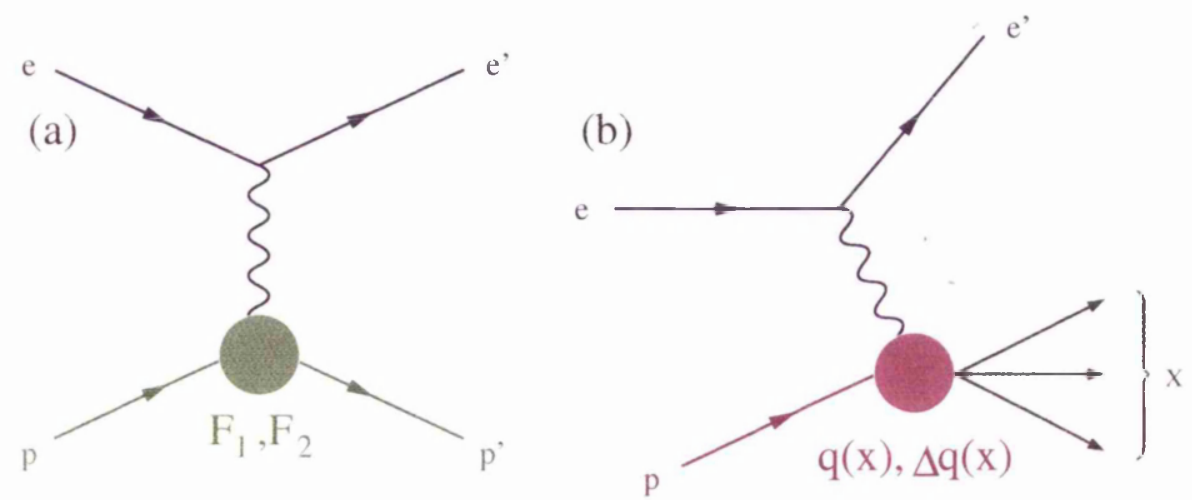

Figure 2.1: Leading order Feynman diagrams for (a) Elastic ep scattering and (b) Deep inelastic scattering. In each respective case, the unknown aspects of the proton structure are parameterised in terms of the form factors, $F_{1}\left(Q^{2}\right)$ and $F_{2}\left(Q^{2}\right)$, and parton distributions, $q(x)$ and $\Delta q(x)$.

Lorentz invariant generalisation of the momentum transfer:

$$
Q^{2}=4 E E^{\prime} \sin ^{2} \frac{\theta}{2}
$$

which itself corresponds to the invariant mass of the virtual photon. Naturally, the physical situation is further complicated by the proton's extended structure and anomalous magnetic moment, which means that the distribution of both its charge and current will contribute to the scattering. This then leads to the Rosenbluth cross section for elastic ep scattering:

$$
\begin{aligned}
\frac{d \sigma}{d \Omega}=\left(\frac{d \sigma}{d \Omega}\right)_{M o t t} & \left\{F_{1}\left(Q^{2}\right)\right. \\
+\tau & {\left.\left[F_{2}^{2}\left(Q^{2}\right)+2\left(F_{1}^{2}\left(Q^{2}\right)+F_{2}^{2}\left(Q^{2}\right)\right)^{2} \tan ^{2} \frac{\theta}{2}\right]\right\}, }
\end{aligned}
$$

where $\tau=Q^{2} / 4 m^{2}, m$ is the mass of the proton, and the Dirac, $F_{1}\left(Q^{2}\right)$, and Pauli, $F_{2}\left(Q^{2}\right)$, electromagnetic form factors have been introduced. These appear in the scattering amplitude as transition current matrix elements and represent vector and tensor transitions between nucleon states, respectively.

The physical interpretation of these form factors is not immediately obvious: a more intuitive interpretation can be obtained through the introduction of the 
Sachs electric and magnetic form factors:

$$
\begin{aligned}
G_{E}\left(Q^{2}\right) & =F_{1}\left(Q^{2}\right)-\tau F_{2}\left(Q^{2}\right) \\
G_{M}\left(Q^{2}\right) & =F_{1}\left(Q^{2}\right)+F_{2}\left(Q^{2}\right)
\end{aligned}
$$

It transpires that, in the Breit frame (which coincides with the electron-proton centre-of-mass), these relate directly to the underlying charge and magnetisation densities at a particular value of $Q^{2}$. For example, at $Q^{2}=0$ their values give the proton charge and magnetic moments, while their slopes can be used to calculate mean squared radii of the proton. Strong $Q^{2}$ dependencies have been observed in both proton Sachs form factors at currently accessible energies, which itself is direct evidence for the extended charge and current distributions within the proton. The latest value for the mean squared radius is $0.86 \mathrm{fm}[3]$.

\subsection{Inclusive Structure: Parton Distributions}

If the nucleon is truly a composite object, it should be possible to gauge its internal structure by increasing the virtuality ${ }^{1}$ of the incoming photon. In the Bjorken regime (where $Q^{2}, \nu \gg m$ ), inclusive lepton scattering has been the reaction of choice for studying the nucleon because the transferred momentum and energy quickly become large enough to cause break-up of the nucleon, often into multihadronic states. In such a process, known as Deep Inelastic Scattering (DIS), only information on the scattered lepton is available, with the final hadronic state remaining undetected. The leading order diagram for electron-proton DIS is shown in fig. 2.1(b). The corresponding analysis of this type of measurement is similar to the previous example with two significant exceptions:

1. The angle and energy of the scattered electron are not strongly correlated as in the elastic case. This means there is no longer a direct relationship between $Q^{2}$ and $\nu$.

2. The deficit in final-state information means that more complicated proton matrix elements appear in the DIS scattering amplitude. These new DIS structure functions are, consequently, quite distinct from the aforemen-

\footnotetext{
${ }^{1}$ The term virtuality is commonly used to refer to the momentum carried by a particle which is not on its mass shell.
} 
tioned form factors: their physical interpretation is no longer as straightforward, and they depend on both $Q^{2}$ and $\nu$.

Therefore, the cross section for this process involves these two new structure functions, $W_{1}\left(\nu, Q^{2}\right)$ and $W_{2}\left(\nu, Q^{2}\right)$ :

$$
\frac{d^{2} \sigma}{d \Omega d E^{\prime}}=\frac{\alpha_{e m}^{2}}{4 E^{2} \sin ^{4} \frac{\theta}{2}}\left[W_{2}\left(\nu, Q^{2}\right) \cos ^{2} \frac{\theta}{2}+2 W_{1}\left(\nu, Q^{2}\right) \sin ^{2} \frac{\theta}{2}\right] .
$$

It was the measurement of $W_{1}$ and $W_{2}$ at SLAC [4] at the end of the 1960's that uncovered the first evidence that the nucleon consists of structureless particles, and found that the DIS cross section of eq. 2.5 is an incoherent sum of individual elastic scattering cross sections from these constituents. To explain these discoveries and develop the Quark Parton Model (QPM) of the nucleon it is necessary to introduce the Bjorken variable:

$$
x=\frac{Q^{2}}{2 m \nu} .
$$

The data showed that at $Q^{2}$ values above a few $G e V$, the structure functions depend only on this new variable $x$. That is to say:

$$
\begin{aligned}
m W_{1}\left(x, Q^{2}\right) & \rightarrow F_{1}^{q p m}(x), \\
\nu W_{2}\left(x, Q^{2}\right) & \rightarrow F_{2}^{q p m}(x) .
\end{aligned}
$$

Recalling that the structure function for any object with internal structure must be $Q^{2}$-dependent, this observation implies that the virtual photons in DIS must be scattering from point-like, structureless objects inside the proton. These internal particles were given the generic name of partons, it was only later that they came to be associated with the quarks and gluons of QCD. The $Q^{2}$-independent behaviour is the now familiar property of Bjorken scaling [5]: it arises as a consequence of the asymptotic freedom associated with QCD at short distances, and implies that the structure functions are independent of a scale transformation in the particle's mass, energy and momentum.

As for the physical significance of $x$, it becomes clear in the so-called infinite momentum frame, where the nucleon is moving with a momentum approaching $\infty$ in the $z$-direction. In such a frame, relativistic time dilation implies that during the interaction with the virtual photon, the nucleon can be considered as a collection of non-interacting partons (the impulse approximation), each with 
different fractions of the total nucleon longitudinal momentum. For one of these partons to absorb a virtual photon of energy $\nu$ and four-momentum $Q^{2}$, it must carry exactly the momentum fraction $x$. It is then clear that DIS, in addition to providing evidence for internal structure of the proton, can be used to measure the momentum distribution of the quarks (and gluons) inside a fast-moving nucleon (for a review see ref. [6]). The unpolarised parton distributions, $q(x)$ and $\bar{q}(x)$, for spin-1/2 quarks and anti-quarks of a given flavour $q$ can be related to the nucleon structure functions of eq. 2.7 by the relation:

$$
F_{2}^{q p m}(x)=2 x F_{1}^{q p m}(x)=x \sum_{q} e_{q}^{2}(q(x)+\bar{q}(x))
$$

where $e_{q}^{2}$ is the charge of the $q$-flavour quark. The quark and gluon parton distributions have been accurately measured over a wide range of kinematics, and have led to some of the most startling revelations concerning the internal nature of the nucleon. The most significant of these discoveries is the fact that the sum of quark momenta only amounts to around $45 \%$ of the nucleon's total momentum.

If instead of considering the spin-averaged structure functions one introduces the spin-dependent structure function $g_{1}$, it is possible to define the polarised parton distributions $\Delta q(x)$ and $\Delta \bar{q}(x)$ in a similar manner:

$$
g_{1}(x)=\sum_{q} e_{q}^{2}(\Delta q(x)+\Delta \bar{q}(x))
$$

The polarised parton distributions are defined as the difference between quark distributions with spin parallel/anti-parallel to the proton spin $\left(\Delta q(x)=q(x)^{\uparrow}-\right.$ $\left.q(x)^{\downarrow}\right)$. Measurement of these entities led to another, even more remarkable, discovery: the spin of the quarks only contribute about $25 \%$ to the total spin of the nucleon. This spin crisis, as it was called, was discovered at CERN in the EMC experiment [7] and has led to the question: if the quarks only carry a quarter of the nucleon's intrinsic spin, where does the remainder come from? To begin to answer this, the nucleon spin $S_{N}$ must be decomposed into three different components:

$$
S_{N}=\frac{1}{2} \Delta \Sigma+\Delta G+L_{z}=\frac{1}{2}
$$

where $\Delta \Sigma$ is the total spin of the quarks, $\Delta G$ the total spin of the gluons and $L_{z}$ is the quarks' orbital angular momentum. Although measurements have shown that 


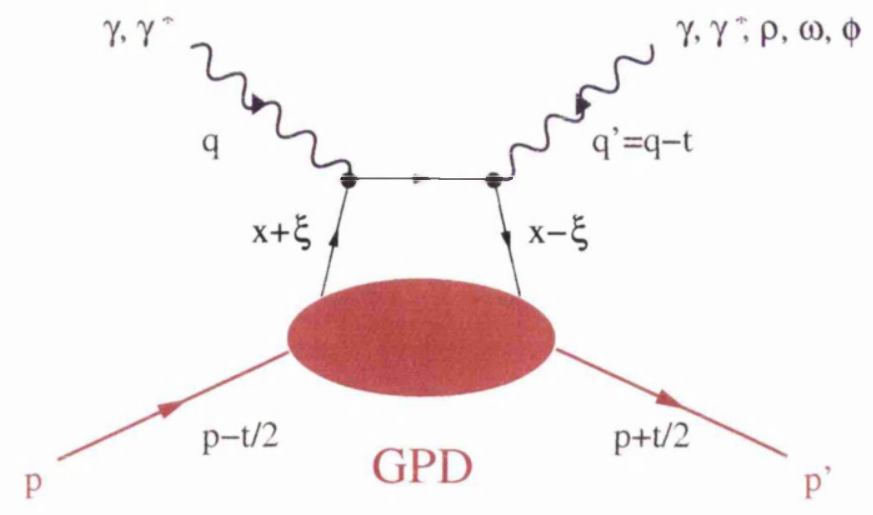

Figure 2.2: The so-called handbag diagram for hard exclusive processes such as real and deeply virtual Compton scattering, or hard meson production; the unknown nucleon structure information is contained within the GPD's.

there are significant contributions from $\Delta G$, it is believed that orbital angular momentum of the quarks will play the dominant role. As we shall see, the issue of quark orbital angular momentum reveals itself time and again in a variety of reactions involving the nucleon.

\subsection{Generalised Parton Distributions}

The two methods of exploring the structure of the nucleon that have been discussed in the previous sections occupy opposing ends of the spectrum of momentum transferred to the proton, and involve quite different theoretical approaches. Recently, attempts have been made to reconcile these somewhat disjointed approaches by introducing Generalised Parton Distributions (GPD). These are reaction independent, hybrid objects related to the non-perturbative structure of hadrons, and have arisen as a tool to describe hard exclusive reactions. In the next chapter we will consider one such process in detail: Compton scattering at moderately high momentum transfer. In this section, therefore, we introduce the GPD's and some of the formalism that accompanies the discussion. Excellent reviews of hard exclusive reactions and GPD's can be found in ref. [8], and more recently in ref. [9].

Hard exclusive processes, like DIS reactions, are dominated by partonic configurations at short, light-like distances, and are usually treated in an infinite momentum frame. One incarnation of the infinite momentum formalism is lightfront dynamics [10], which is particularly suited to reactions of this type since 
momentum transfers can be represented by simple Lorentz boosts, and when dealing with composite objects such as the nucleon, there is a natural separation of the centre-of-mass motion from the intrinsic relative motion of the partons. Specifically for a high energy nucleon, this implies that the longitudinal and transverse degrees of freedom of the constituents are naturally separated. For example, a particle with four-momentum $p^{\mu}=\left(p^{0}, p^{1}, p^{2}, p^{3}\right)$ can be represented in a system with momentum along the $p^{3}$ axis approaching $\infty$, such that:

$$
p^{ \pm}=p^{0} \pm p^{3}=\text { constant }
$$

In this system, the four-momentum becomes $p^{\mu}=\left(p^{+}, p^{-}, \mathbf{p}_{\perp}\right)$, with momenta on the light-front surface of $p^{ \pm}$, and transverse momenta $\mathbf{p}_{\perp}=\left(p^{1}, p^{2}\right)$.

A typical hard exclusive Feynman diagram, for processes such as real and virtual Compton scattering or meson production, can be seen in fig. 2.2. As with the previous two examples, a parameterisation is introduced to account for the unknown nucleon structure information: the GPD's. Formally, these are Fourier transforms of nucleon matrix elements which relate quark-gluon and nucleon degrees of freedom. At twist-2 level there are four chirality-conserving quark GPD's, each of which represents a different contribution to the overall nucleon structure: $H$ and $E$ (unpolarised), and $\tilde{H}$ and $\tilde{E}$ (polarised). Twist, itself, is a fairly abstract concept, related to the operators that appear in the nucleon matrix elements and the logarithmic evolution of a physical system. A formal definition can be found in ref. [1], although it is probably sufficient for our purposes to note that diagrams involving quark propagators are at twist-2 (or leading twist) level. In addition to the fraction of the nucleon's momentum carried by the particular quark, $x$, the GPD's depend on the Mandelstam invariant momentum transfer $t$ and the skewedness parameter $\xi=-t^{+} / 2 p^{+}$, which is simply the light-cone momentum fraction transferred to the target nucleon in fig. 2.2 .

The real power of this approach is revealed in model-independent sum rules, which lead to an intimate relation between the GPD's and matrix elements of local currents, such as the electromagnetic form factors. For example, the contribution to the electromagnetic form factors from a particular quark flavour, $q$, are given 
by

$$
\begin{aligned}
& \int_{-1}^{+1} d x H^{q}(x, \xi, t)=F_{1}^{q}(t), \\
& \int_{-1}^{+1} d x E^{q}(x, \xi, t)=F_{2}^{q}(t), \\
& \int_{-1}^{+1} d x \tilde{H}^{q}(x, \xi, t)=g_{A}^{q}(t), \\
& \int_{-1}^{+1} d x \tilde{E}^{q}(x, \xi, t)=h_{A}^{q}(t) .
\end{aligned}
$$

These can simply be related to the full nucleon form factors using $\mathrm{SU}(2)$ isospin symmetry. $F_{1}$ and $F_{2}$ are the Dirac and Pauli form factors introduced in eq. 2.3, and $g_{A}$ and $h_{A}$ are proton form factors which arise in weak, rather than electromagnetic, currents. Furthermore, $H$ and $\tilde{H}$ can be linked to the ordinary parton distributions of DIS in the forward limit $(\xi=t=0)$ :

$$
\begin{aligned}
& H^{q}(x, 0,0)=\left\{\begin{array}{cc}
q(x), & x>0, \\
-\bar{q}(-x), & x<0 .
\end{array}\right. \\
& \tilde{H}^{q}(x, 0,0)=\left\{\begin{array}{cc}
\Delta q(x), & x>0, \\
\Delta \bar{q}(-x), & x<0 .
\end{array}\right.
\end{aligned}
$$

However, this is not the case for $E$ and $\tilde{E}$, which are not accessible in DIS since these tensors disappear in the forward limit. An example of the connection between GPD's and ordinary parton distributions can be seen in the parameterisation of the $u$-quark GPD $H(x, \xi, t=0)$ in fig. 2.3. Models of the GPD's such as this one are all that are available at present, since the monumental task of experimentally mapping out these complicated objects has only just begun.

The role of the GPD's as a bridge between the already available inclusive and exclusive descriptions of the nucleon is plainly established in eq. 2.12 and 2.13. More than simply this, however, the GPD's and the hard exclusive processes which they describe have the potential to reveal a great deal more about the nucleon. It is in comparison with the ordinary parton distributions that this becomes clear. In DIS, there is no overlap between initial and final states, the parton distributions arise through diagonal nucleon matrix elements and are purely number densities. GPD's, on the other hand, are probability amplitudes that represent interference between initial and final states (one state with a wave function which 


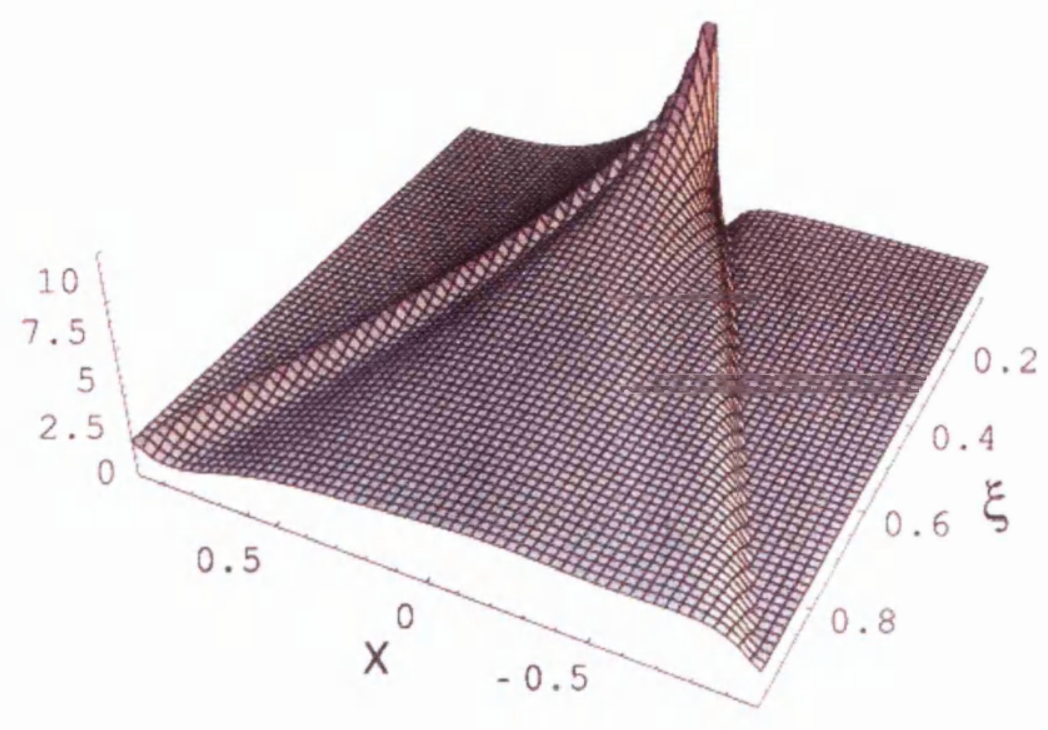

Figure 2.3: Parameterisation of the $G P D H^{u}(x, \xi, t=0)$ from ref. [8]. At $\xi=0$ the distribution reduces to $q(x)$ and $q \overline{(}-x)$ as given in eq. 2.13 .

involves a parton with momentum $x+\xi$ and one with $x-\xi)$ and, therefore, correlate different partonic configurations at the quantum mechanical level ${ }^{2}$. Where DIS involves only longitudinal momentum fractions, GPD's contain information on the transverse momentum of partons inside a fast moving nucleon, involve quark-quark momentum correlations and are sensitive to configurations involving quark and anti-quarks.

In addition, an examination of the polarised structure of the GPD's reveals that $H$ and $\tilde{H}$ are purely nucleon helicity-conserving, whereas $E$ and $\tilde{E}$ are naturally associated with nucleon helicity-flip amplitudes. Fast moving fermions, such as quarks, do not change helicity which means that nucleon helicity-flip requires configurations with non-zero quark orbital angular momentum. This is essentially stated in Ji's angular momentum sum rule [11], which is written as:

$$
\int_{-1}^{+1} d x x\left(H^{q}(x, \xi)+E^{q}(x, \xi)\right)=2 J^{q},
$$

where, $J^{q}$ is the fraction of the nucleon's angular momentum (sum of spin and orbital) carried by a quark of flavour $q$. This relationship is intimately related to the proton spin crisis and the decomposition of the nucleon spin given in eq. 2.10. Quark orbital angular momentum and nucleon helicity-flip prove to be

\footnotetext{
${ }^{2}$ This is the reason they are also referred to as off-diagonal or skewed parton distributions.
} 
very important components of calculations involving a variety of hard exclusive reactions: in the following chapter, we will see that this is certainly true of proton Compton scattering. 


\section{Chapter 3}

\section{Proton Compton Scattering}

In the previous chapter we have seen the real power of electromagnetic probes, in particular the virtual photon, and the wealth of nucleon structure information they make available. We now turn our attention to the case of elastic scattering of real photons from proton targets: $\gamma p \rightarrow \gamma^{\prime} p$. Arthur Compton first observed the phenomenon which bears his name in experiments using X-rays, and the interpretation of the observations in terms of scattering of a single quantum of electromagnetic radiation is one of the highlights in early quantum theory. Subsequently, Klein and Nishina [12] derived the cross section at leading order QED in what has become a standard textbook example. This chapter begins with an overview of Compton scattering at comparatively low momentum transfer, where an abundance of data has led to a greatly enhanced understanding of the electromagnetic response of the proton. We then go on to discuss several calculations at large momentum transfer, in a region where access to the composite structure of the proton is made possible but the experimental data is much more sparse.

\subsection{Low Energy Regime}

Measurements of real Compton scattering from the proton in the region of around $100 \mathrm{MeV}$ to $2 \mathrm{GeV}$ have fundamentally enhanced our understanding of the nature of the proton and its excited states. Just as low momentum virtual photons reveal the size and shape of the nucleon, low energy real photons can be used to investigate the response of the nucleon to long wavelength, low frequency electromagnetic fields. This naturally leads to the measurement of two elementary physical quantities: the proton's electric $\left(\alpha_{E}\right)$ and magnetic $\left(\beta_{M}\right)$ polarisibilities. These are closely related to the field of dispersion theory, from which sum rules 
can be obtained leading to a model independent analysis of the experimental data. Sum rules are invaluable as they directly connect low energy properties of the proton to the photoabsorption cross section. An excellent review of dispersion relations, sum rules and Compton scattering is that of Drechsel et al. [13].

\section{Proton Polarisibilities}

The adopted model of intrinsic nucleon structure introducing departures from point-like behaviour is once again the foundation on which the discussion is based. Essentially, evaluation of the difference between the point-like and observed unpolarised Compton cross sections allows one to determine the proton polarisibilities through the Baldin sum rule [14]:

$$
\alpha_{E}+\beta_{M}=\frac{1}{2 \pi^{2}} \int_{\omega_{0}}^{\infty} \frac{d \omega}{\omega^{2}} \sigma_{t o t}(\omega),
$$

where $\omega_{0}$ is the pion production threshold energy. Precisely how this relationship between polarisibilities and cross section arises can be understood in terms of the following considerations:

1. An expansion of the Compton amplitude in powers of photon energy, $\omega$, introduces deviations connected with the two scalar polarisibilities at $\mathcal{O}\left(\omega^{2}\right)$ (incidentally, an additional four vector (spin) polarisibilities appear at higher order).

2. A dispersion relation analogous to the Kramers-Kronig relation from optics can be used to connect the real part of the Compton amplitude to a dispersion integral over its imaginary part.

3. Unitarity considerations within the optical theorem then connect the imaginary part of the amplitude with the total photon-proton absorption cross section.

The significance of the last point, in particular, is worth emphasising further, as the connection between the total cross section and the proton's excitation spectrum leads to a close relationship between Compton scattering, nucleon spectroscopy and meson production in the resonance regime.

Small values have been measured for the scalar polarisibilities [15], which implies that the proton is a rather rigid object that does not deform significantly under the influence of an external electromagnetic field. The electric polarisibility 
has been found to be much larger than its magnetic counterpart. This difference has been attributed to cancellation between various magnetic contributions to $\beta_{M}$.

\section{Spin Structure and the GDH Sum Rule}

Similar arguments can be employed when considering the case of circularly polarised photons on polarised proton targets, leading to the Gerasimov-Drell-Hearn (GDH) sum rule $[16,17]$. In this case, the Compton scattering amplitude can be separated into two parts: a non-flip amplitude $\left(f_{3 / 2}\right)$, corresponding to proton polarisation parallel to the that of the photon; and a spin-flip part $\left(f_{1 / 2}\right)$ for the case of anti-parallel polarisations. The GDH sum rule then relates the proton's anomalous magnetic moment, $\kappa$, to the difference between total flip and non-flip cross sections:

$$
\frac{\alpha_{e m} \kappa^{2}}{m^{2}}=\frac{1}{2 \pi^{2}} \int_{\omega_{0}}^{\infty} \frac{d \omega}{\omega}\left[\sigma_{3 / 2}(\omega)-\sigma_{1 / 2}(\omega)\right]
$$

In this sense, the GDH sum rule provides complementary spin structure information at low energy to the polarised parton distributions of DIS. This is one of the reasons that there are significant efforts currently underway to generalise the sum rule to describe virtual photon scattering, therefore allowing extension into a much higher energy regime where partonic configurations of the proton become important.

\subsection{Asymptotically Large Energies: pQCD}

The observation that the onset of scaling in DIS occurs at momentum transfers of only a few $\mathrm{GeV}$ led, quite reasonably, to speculation that similar behaviour might be expected in exclusive reactions on a similar energy scale. The pioneering work of Brodsky and Lepage [10] laid the foundations for a treatment of hard exclusive reactions in a PQCD framework. There is general consensus that calculations of this type will provide the appropriate description of exclusive reactions at very high energies. At present, however, the applicability of this approach to data at moderate energies is the subject of considerable debate. 


\subsubsection{Leading Twist Factorisation}

In this approach, the scattering amplitude of exclusive processes at large momentum transfer is assumed to factorise into hard and soft subprocesses, with the physical amplitude being governed largely by quark and gluon interactions at short distances. The first and underlying assumption is that the energy scale is sufficiently high for the partons' mass and transverse momenta to be neglected. Moreover, such a large scale implies that only the valence-quark states of the hadron contribute, with non-valence states involving sea quark and gluon contributions being strongly suppressed. The physical situation, therefore, is of valencequarks moving collinear with the parent hadron which must remain in its ground state, and therefore momentum must be transferred explicitly between quark lines by the minimum allowable number of hard gluon exchanges. Schematically, the amplitude can be considered as:

$$
T=\phi_{\text {initial }} \otimes T_{H} \otimes \phi_{\text {final }}
$$

where $\phi_{\text {initial }}$ and $\phi_{\text {final }}$ are the hadron's parton Distribution Amplitudes (DA) wherein the non-perturbative physics is encoded. These are process independent quantities, analogous in many respects to parton distributions in DIS, and represent formally the probability of finding a hadron with a specific partitioning of the valence-quarks' longitudinal momenta. The hard subprocess amplitude $T_{H}$, whereby the momentum is transferred to the hadron, is obtained by calculating the Feynman diagrams for the specific hard process.

These considerations lead to two of the most familiar and rigorous predictions of pQCD which can be tested experimentally: Hadron Helicity Conservation (HHC) [18] and constituent counting rules [19]. The assumptions of zero quark mass and dominant valence configurations of the hadrons, together with the vector coupling of the gauge particles, leads to the phenomenon of conservation of hadron helicity in hard exclusive reactions. The constituent counting rules, on the other hand, arise because a system of bound partons under the transfer of large momentum has the same dimensional scaling behaviour (due to asymptotic freedom) as if it were a collection of free particles. This is usually written as:

$$
\frac{d \sigma}{d t}=s^{2-n} f\left(\theta_{c m}\right)
$$

where $n$ is the total number of leptons, photons and quark constituents in the 


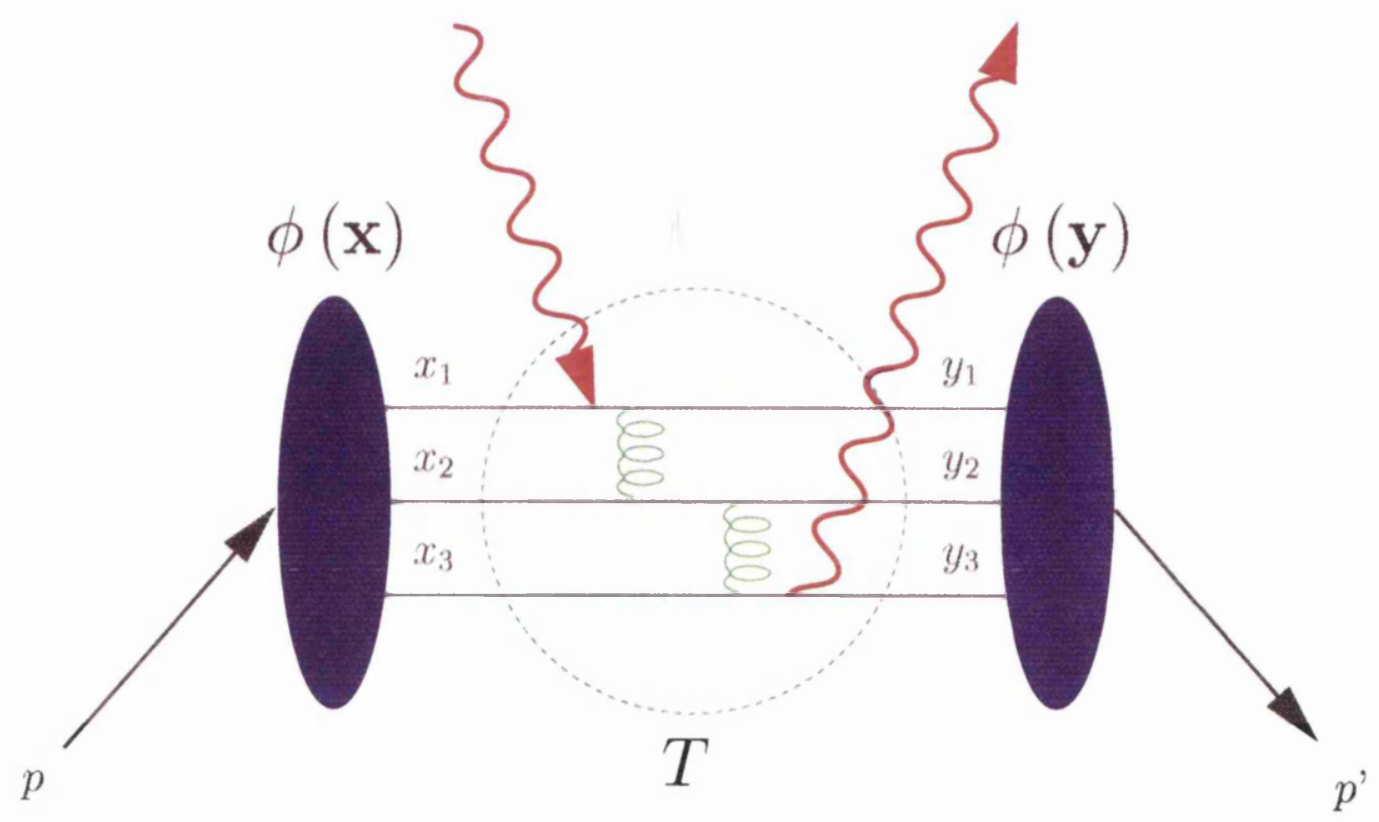

Figure 3.1: Leading twist factorisation of exclusive Compton scattering, in which momentum is shared between three active valence quarks by the exchange of two hard gluons.

initial and final states, which represent the elementary field components in the amplitude of a given process ${ }^{1}$.

\subsubsection{Calculations for Compton Scattering}

There have been several recent attempts within the above framework to evaluate exclusive Compton scattering to leading twist accuracy and leading order in $\alpha_{s}$. The original work by Farrar, Zhang et al. $|20-22|$ showed that leading order pQCD predictions were safe from singularities that might otherwise require resummation of the perturbative series. There has since been three subsequent calculations $[23-25]$, the last two including predictions for spin observables. Technically, the calculations are quite involved since a great many diagrams contribute, even at leading order, and the predictions are sensitive to both the renormalisation scale dependence and the treatment of singularities in the hard scattering amplitude.

All the calculations involve factorisation of the type given in eq. 3.3, which is best visualised by the Feynman diagram of fig. 3.1. As one would expect, the amplitude involves a hard scattering subprocess $(\gamma q q q \rightarrow \gamma q q q)$ with two

\footnotetext{
${ }^{1}$ For Compton scattering, $n=8$.
} 
hard gluons exchanged between the active quarks, and soft matrix elements in the form of proton distribution amplitudes. If $h, h^{\prime}= \pm \frac{1}{2}$ are the helicities for the incoming and outgoing protons and $\lambda, \lambda^{\prime}= \pm 1$ for incoming and outgoing photons, the helicity amplitudes for the process take the form:

$$
\begin{array}{r}
\mathcal{M}_{h h^{\prime}}^{\lambda \lambda^{\prime}}=\sum_{d, i} \int_{0}^{1} d^{3} \mathbf{x} d^{3} \mathbf{y} \delta\left(1-\sum_{j=1}^{3} x_{j}\right) \delta\left(1-\sum_{k=1}^{3} y_{k}\right) \\
\times \phi_{i}(\mathbf{x}) T_{i}^{(d)}\left(\mathbf{x}, h, \lambda ; \mathbf{y}, h^{\prime}, \lambda^{\prime}\right) \phi_{i}^{*}(\mathbf{y})
\end{array}
$$

where $i$ labels the independent, three-valence-quark Fock states of the proton and the sum over $d$ represents all Born level diagrams contributing to the hard scattering amplitude, $T_{i}$. The proton DA's, assuming only collinear valence quarks in the initial and final states, are $\phi_{i}(\mathbf{x})$ and $\phi_{i}(\mathbf{y})$, respectively. The longitudinal momentum fractions are given by $\mathbf{x} \equiv\left(x_{1}, x_{2}, x_{3}\right)$ and $\mathbf{y} \equiv\left(y_{1}, y_{2}, y_{3}\right)$, with the delta functions in the convolution integral ensuring that these sum to unity.

The DA's in these calculations are defined as the integral over transverse momenta of the three-quark-component of the proton Light-Cone Wave Function (LCWF). We postpone a full discussion of the Fock expansion of the proton and its interpretation in terms of light-cone wave functions until sec. 3.3.2, simply noting here that the form of the DA's is not known a priori but has to rely on models of the wave functions, of which several are available. The three most common DA models used to make predictions for Compton scattering are based on QCD sum rule analyses and labeled CZ [26], KS [27] and COZ [28]. Their most interesting, and controversial, feature is the dominance of configurations where one of the quarks carries most of the momentum; that is to say, they are strongly concentrated at the endpoint regions, $x \simeq 0$ and $x \simeq 1$. The applicability of a perturbative approach in these endpoint regions is questionable and has been the source of some criticism [29].

The integrals over the quark longitudinal momentum fractions in eq. 3.5 are evaluated by computing each of the 336 leading order diagrams. Complications arise when one or more of the internal lines in a particular diagram (i.e. the quark propagators) goes on the mass shell, resulting in singularities in the amplitude. The different treatments of these singularities in the numerical integration have led to significant discrepancies between the theoretical predictions [25], even when the same DA's are used. 

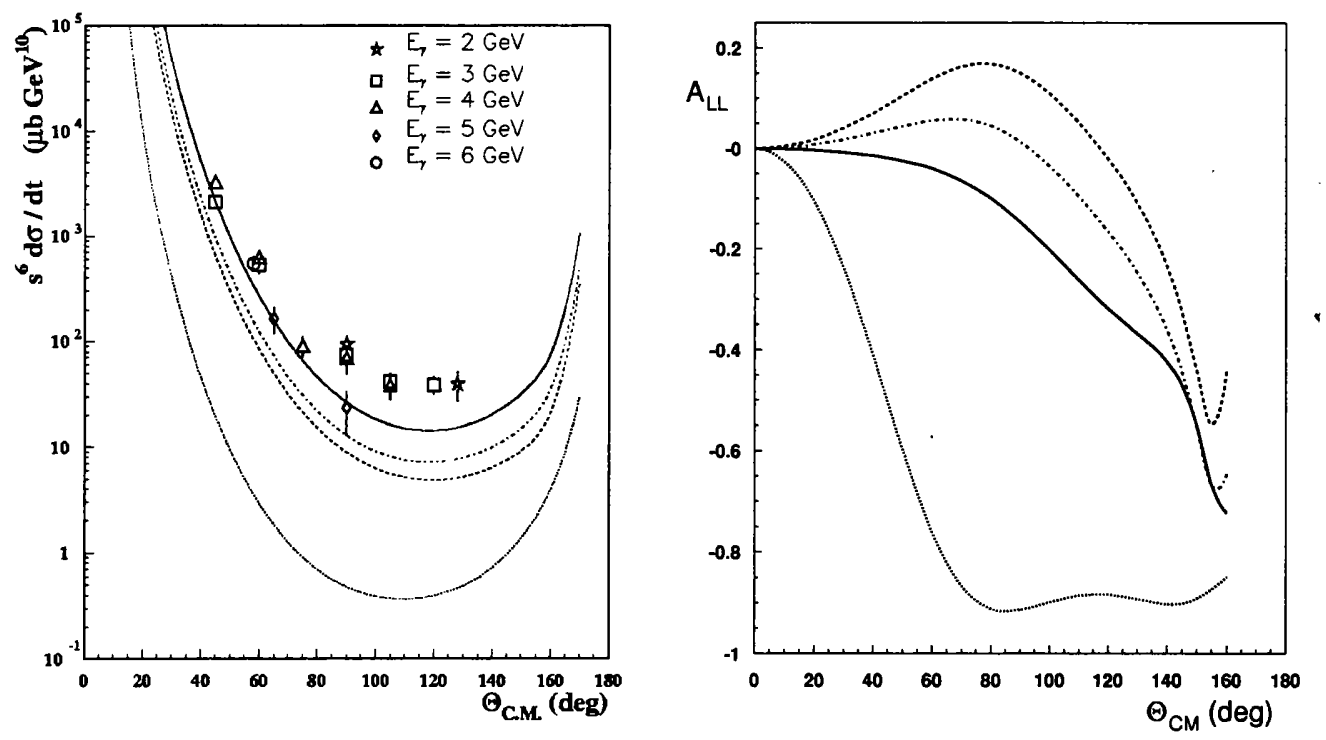

Figure 3.2: $p Q C D$ predictions from ref. [24] for differential cross section (left) compared with Cornell data [30], and spin observable $A_{L L}$ (right). The various curves correspond to different DA's: CZ [26] (dashed line), KS [27] (full line), COZ [28] (dashed-dotted line) and asymptotic (dotted line).

\subsubsection{Predictions and Comparison with Available Data}

The calculation of specific Compton scattering observables from the helicity amplitudes of eq. 3.5 is simplified by the fact that many of the amplitudes reduce to zero. For example, HHC implies that

$$
\mathcal{M}_{\uparrow \downarrow}^{\lambda \lambda^{\prime}}=0
$$

Moreover, consideration of parity and time-reversal invariance further reduces the number of independent amplitudes to three, where $\mathcal{M}_{\uparrow \uparrow}^{\uparrow \uparrow}, \mathcal{M}_{\uparrow \uparrow}^{\uparrow \downarrow}, \mathcal{M}_{\uparrow \uparrow}^{\Downarrow \downarrow}$ are chosen.

Before discussing the comparison of theory with the single relevant data-set in existence, it is important to stress that there is widespread acceptance that the photon energy at which these data were taken [30], and of the present measurement, might be too low for perturbative calculations to achieve full accuracy. Nevertheless, several pQCD predictions have been made for the differential cross section at these relatively low photon energies. Those from ref. [24] are displayed on the left plot of fig 3.2 , which shows the cross section scaled by $s^{6}$ and the predictions of various model wave functions. As well as the three models discussed 
above, the asymptotic DA, based upon a non-physical proton wave function where the quarks are treated as essentially free particles within the proton, is also included. The inclusion of these four separate predictions highlights the overall sensitivity to the particular form of the proton wave function, which at these energies is significant.

As for comparison with data, at wide angles there is reasonable agreement, albeit with limited statistical accuracy, with the constituent scaling behaviour of eq. 3.4. The cross section data, however, are more than an order of magnitude higher than the closest model prediction (KS). More generally, it has long since been the case that constituent scaling appears to hold for a wide range of reaction types at moderately high energy and momentum transfer. That in itself has been sufficient for many observers to argue in favour of the applicability of the pQCD approach. However, a wealth of polarisation data - from reactions as diverse as proton-proton elastic scattering [31], charmonium decay [32], and recent Jefferson Lab results on elastic form factors [33,34] and neutral pion photoproduction [35] - have shown clear violation of HHC. This has led to speculation that the leading twist factorisation will only become dominant at higher energies $(\sim 50-100 \mathrm{GeV})$, and an alternative theoretical framework might be needed for currently accessible energies.

\section{Proton Spin Observables}

In addition to making predictions on the spin-averaged differential cross section, the helicity amplitudes of eq. 3.5 can also be used to calculate various spin observables for Compton scattering. The motivation for examining these should be apparent in light of the aforementioned recent observations of HHC violation. The proton spin observables are frame dependent (because of the non-zero proton mass) and must be defined in a particular reference frame. We adopt the CM coordinate system defined in ref. [36,37]:

$$
\begin{aligned}
\mathbf{L} & =\frac{\hat{p^{\prime}}}{\left|\hat{p^{\prime}}\right|}, \\
\mathbf{N} & =\hat{q} \times \hat{p^{\prime}}, \\
\mathbf{T} & =\mathbf{N} \times \hat{p^{\prime}},
\end{aligned}
$$




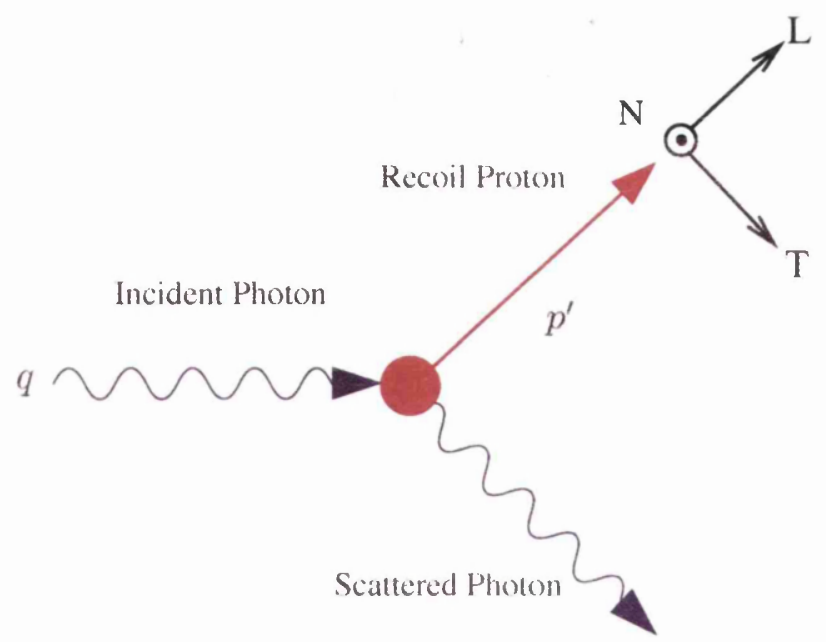

Figure 3.3: Definition of proton spin observables in the CM coordinate system. The axes $\mathbf{L}, \mathbf{N}$ and $\mathbf{T}$ correspond to the longitudinal, normal and transverse directions defined in eq. 3.7.

where $\mathbf{L}, \mathbf{N}$ and $\mathbf{T}$ define the longitudinal, normal and transverse ${ }^{2}$ spin directions; and $\hat{q}$ and $\hat{p}^{\prime}$ are the directions of the incident photon and recoil proton, as demonstrated in fig. 3.3.

The present measurement of the polarisation transfer in the $\vec{\gamma} p \rightarrow \gamma \vec{p}$ reaction involves two-spin correlations between initial photon (first arrow in eq. 3.8) and recoil proton (second arrow). These are defined by the relations:

$$
\begin{aligned}
K_{L L} \frac{d \sigma}{d t} & =\frac{d \sigma(\uparrow \uparrow)}{d t}-\frac{d \sigma(\downarrow \uparrow)}{d t}, \\
K_{L T} \frac{d \sigma}{d t} & =\frac{d \sigma(\uparrow \rightarrow)}{d t}-\frac{d \sigma(\downarrow \rightarrow \rightarrow)}{d t} \\
K_{L N} & =0 .
\end{aligned}
$$

The normal component of the proton polarisation transfer in Compton scattering, $K_{L N}$, is zero because of parity conservation. Predictions involving $K_{L L}$ and $K_{L T}$ are complicated by the fact that theoretical calculations routinely involve correlations between the incoming photon and initial-state proton. The predictions for the longitudinal initial-state spin correlation, $A_{L L}$, using the same DA's as in the case of the cross section, can be seen on the right of fig. 3.2. In the strict absence of proton helicity-flip, as in the $\mathrm{pQCD}$ approach, it is trivial to recover

\footnotetext{
${ }^{2}$ In ref. [37] the transverse component is referred to as the sideways polarisation (S).
} 
the experimentally important polarisation transfer because

$$
K_{L L}=A_{L L} .
$$

This same constraint implies that the transverse polarisation transfer is zero:

$$
K_{L T}=A_{L T}=0 .
$$

\subsection{The Handbag Factorisation}

An alternative to leading twist factorisation is the so-called handbag factorisation, in which a single quark absorbs all of the momentum: a property implicit in the endpoint-concentrated DA's in the pQCD calculations. It was first considered in 1969 by Bjorken and Paschos [38], and has enjoyed a revival of late in calculations involving meson production [39], two-photon annihilation into hadrons [40] and both real and virtual Compton scattering [41]. This factorisation differs significantly from the pQCD approach, as the momentum transferred to the single active quark is shared amongst the other constituents of the proton through overlaps of non-perturbative proton wave functions, which represent countless soft gluon exchanges.

From the outset it should be stated that the handbag mechanism is not an opposing or mutually-exclusive formalism to the leading twist factorisation, but rather it represents a power correction to leading twist. In principle, there are many possible mechanisms for Compton scattering, whose relative contribution to the amplitude depends on the particular kinematic regime. At asymptotically high energy, the leading twist mechanism dominates since all others - such as processes involving one-hard gluon exchange, the handbag diagram or other non-valence configurations of the proton - are suppressed by inverse powers of the hard scale. However, at moderate momentum transfer these other processes become much more significant. To fully assess the reaction amplitude when leading twist does not dominate, a coherent sum of all these contributions must be made. This is currently impossible within a QCD framework since each contribution has a different associated soft proton matrix element, incalculable in QCD. Instead, phenomenological analyses are employed to find if any particular mechanism dominates in specific kinematic regions. 

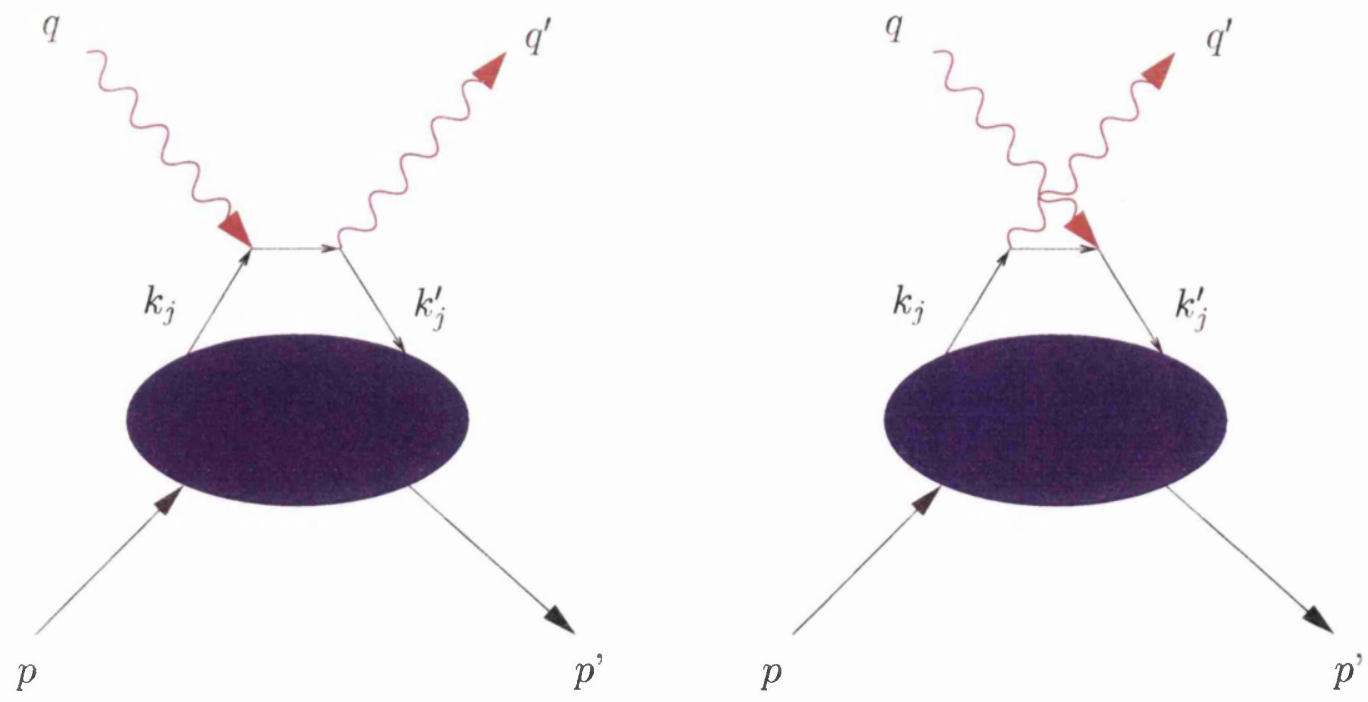

Figure 3.4: Born and crossed Born diagrams for exclusive Compton scattering in the handbag factorisation. Proton, photon and parton momenta are given by $p, q$ and $k$, respectively

\subsubsection{The Soft Overlap Mechanism}

It is within the handbag factorisation that Compton scattering phenomenology connects to the generalised parton distribution formalism and, specifically, to the interpretation of GPD's in terms of the overlap of soft proton wave functions. Radyushkin [41] first described this overlap in terms of the double distribution representation of GPD's, and was closely followed by Diehl, Kroll et al. who used a Fock state expansion of the proton for the wave function parameterisation $[37$, $42-44 \mid$.

The calculations involve the Born and crossed Born diagrams shown in fig. 3.4. As in the previous instance, the amplitude is factorised: in this case, into a hard photon-parton level subprocess $(\gamma q \rightarrow \gamma q)$ calculable in pQCD, and soft proton matrix elements representing the emission and re-absorption of a single parton, which, like the DA's discussed previously, are obtained from proton wave functions. The essential condition for the amplitude to factorise is a large transverse momentum transfer to the proton, satisfied when the Madelstam variables $(s,-t$ and $-u$ ) are large on a typical hadronic scale $\Lambda$ (in the $\mathrm{GeV}$ region). The key points inherent in this handbag approach are then:

- The emission and re-absorption vertices are assumed to be bound by a soft scale. Put another way, the proton wave functions are dominated by 
(spectator) partons with small virtualities and small intrinsic transverse momenta, since large intrinsic transverse momenta are characteristic of hard gluon exchange. Therefore, the inequalities for the spectator parton momenta

$$
\begin{aligned}
k_{i}^{2} & <\Lambda^{2}, \\
\mathbf{k}_{\perp \mathbf{i}}^{2} / x_{i} & <\Lambda^{2},
\end{aligned}
$$

must hold, where the light-cone momentum fraction is $x_{i}=k_{i}^{+} / p^{+}$.

- It is further assumed that the active quark, i.e. the one involved in the hard scattering subprocess, is approximately on-shell, moves collinear with the proton and carries a momentum fraction close to unity $\left(x_{j}=x_{j}^{\prime}=1\right)$.

- The calculations are performed within a symmetric reference frame where the positive light-cone components of the incoming and outgoing proton momenta are the same. This implies zero skewedness:

$$
\xi=\frac{\left(p-p^{\prime}\right)^{+}}{\left(p+p^{\prime}\right)^{+}}=0
$$

Therefore, using the same helicity convention as in sec. 3.2.2, the proton non-flip and flip amplitudes are:

$$
\begin{aligned}
& \mathcal{M}_{\uparrow \uparrow}^{\lambda \lambda^{\prime}}(s, t)=2 \pi \alpha_{e m} {\left[\mathcal{H}_{\uparrow \uparrow}^{\lambda \lambda^{\prime}}(s, t)\left(R_{V}(t)+R_{A}(t)\right)\right.} \\
&+\left.\mathcal{H}_{\downarrow \downarrow}^{\lambda \lambda^{\prime}}(s, t)\left(R_{V}(t)-R_{A}(t)\right)\right], \\
& \mathcal{M}_{\uparrow \downarrow}^{\lambda \lambda^{\prime}}(s, t)=\pi \alpha_{e m} \frac{\sqrt{-t}}{m}\left[\mathcal{H}_{\uparrow \uparrow}^{\lambda \lambda^{\prime}}(s, t)+\mathcal{H}_{\downarrow \downarrow}^{\lambda \lambda^{\prime}}(s, t)\right] R_{T}(t) .
\end{aligned}
$$

Here, the amplitudes for the hard kernel are $\mathcal{H}(s, t)$, which are real at leading order in $\alpha_{s}$, involve no quark helicity-flip and can be reduced $\mathrm{to}^{3}$

$$
\mathcal{H}_{\uparrow \uparrow}^{\uparrow \uparrow}=2 \sqrt{\frac{s}{-u}}, \quad \mathcal{H}_{\downarrow \downarrow}^{\uparrow \uparrow}=2 \sqrt{\frac{-u}{s}}, \quad \mathcal{H}_{\downarrow \uparrow}^{\uparrow \uparrow}=0
$$

The soft physics, on the other hand, is encoded in the new proton form factors,

\footnotetext{
${ }^{3}$ Note that the bottom arrows in the hard subprocess amplitudes refer to quark, not proton, helicty.
} 
$R_{\iota}(t)(\iota=V, A, T)$. These are defined as $1 / x$ moments of the corresponding GPD's at $\xi=0$, which makes them quite distinct from the electromagnetic form factors which are $x^{0}$ moments (eq. 2.12). Although often referred to as the Compton form factors, they are not unique to Compton scattering but appear in a variety of processes, such as those mentioned in relation to the handbag factorisation at the beginning of this section. For example, their diversity is highlighted by extension into the time-like region, where they appear in twophoton annihilation calculations. The form factors are defined for a quark of flavour $a=(u, d, \ldots)$ by

$$
\begin{aligned}
& R_{V}^{a}(t)=\int_{-1}^{1} \frac{d \bar{x}}{\bar{x}} H^{a}(\bar{x}, 0 ; t), \\
& R_{A}^{a}(t)=\int_{-1}^{1} \frac{d \bar{x}}{\bar{x}} \operatorname{sign}(\bar{x}) \widetilde{H^{a}}(\bar{x}, 0 ; t), \\
& R_{T}^{a}(t)=\int_{-1}^{1} \frac{d \bar{x}}{\bar{x}} E^{a}(\bar{x}, 0 ; t),
\end{aligned}
$$

where the light-cone momentum fraction associated with the active quark is $\bar{x}=$ $\left(k_{j}+k_{j}^{\prime}\right)^{+} /\left(p+p^{\prime}\right)^{+}$. The full proton form factors can be obtained from a sum over quark flavour $a$, weighted by the square of the quark charge $e_{a}$ :

$$
R_{\iota}(t)=\sum_{a} e_{a}^{2} R_{\iota}^{a}(t)
$$

There is no contribution from the pseudo-scalar GPD $\widetilde{E}$ to Compton scattering in the symmetric reference frame.

\subsubsection{The Modelling of Form Factors}

Since the experimental observables depend upon the exact form of the $R_{\iota}$ form factors, they (or alternatively the underlying GPD's) have to be modelled before any quantitative predictions can be made. One method [45] is to represent the form factors as overlaps of soft light-cone wave functions in momentum space, summed over all Fock states of the proton. Let us begin this discussion by defining formally what is meant by the terms soft wave functions and valence Fock states.

Any hadronic state can be represented by a superposition of the more fundamental partonic Fock states, which appear as a direct consequence of the ele- 
mentary quark-gluon field. A simplistic Fock state decomposition of the proton might take the form:

$$
|P\rangle=\Psi_{V}|q q q\rangle+\Psi_{q \bar{q}}|q q q, q \bar{q}\rangle+\Psi_{g}|q q q, g\rangle+\ldots
$$

where the $\Psi_{i}(i=V, q \bar{q}, \ldots)$ are Light Cone Wave Functions (LCWF) of the proton, through which the proton state naturally couples to the ith Fock state. For a particular N-parton Fock state, the LCWF (such as $\Psi_{V}$ for the $N=3$ valence state) depends on parton momenta relative to the proton, as well as the flavour, color and spin of the partons which make up the state.

For the purpose of parameterising the GPD's, only the soft parts of the LCWF's are important. This implies removal of the perturbative tails of the wave functions, which are associated with large intrinsic transverse momenta and, therefore, with hard gluon exchange. One can then assume a simple LCWF with a Gaussian form for the transverse parton momenta $\mathbf{k}_{\perp \mathbf{i}}$, which is consistent with the fundamental assumptions of eq. 3.11 :

$$
\Psi_{N, \beta}\left(x_{i}, \mathbf{k}_{\perp \mathbf{i}}\right) \propto \exp \left[-\alpha^{2} \sum_{i=1}^{N} \frac{\mathbf{k}_{\perp \mathbf{i}}^{\mathbf{2}}}{x_{i}}\right]
$$

$\beta$ represents the spin-flavour combination and $\alpha$ the transverse size parameter of the particular Fock state. The latter tuns out to be the only free parameter in the resultant model, and even this is limited to the physical range 0.8 to $1.2 \mathrm{GeV}^{-1}$.

The vector and axial-vector form factors can then be represented by an overlap integral of the wave functions of eq. 3.19. The resultant formulae (eq. 3.20) highlight the clear link between GPD's and ordinary parton distributions as expressed in eq. 2.13, with $R_{V}(t)$ and $R_{A}(t)$ reducing to $q(x)$ and $\Delta q(x)$ :

$$
\begin{aligned}
& R_{V}(t)=\sum_{a} e_{a}^{2} \int \frac{d \bar{x}}{\bar{x}} \exp \left[\frac{1}{2} \alpha^{2} t \frac{1-\bar{x}}{\bar{x}}\right] q_{a}(\bar{x}), \\
& R_{A}(t)=\sum_{a} e_{a}^{2} \int \frac{d \bar{x}}{\bar{x}} \exp \left[\frac{1}{2} \alpha^{2} t \frac{1-\bar{x}}{\bar{x}}\right] \Delta q_{a}(\bar{x}) .
\end{aligned}
$$

If the transverse size parameter is taken to be equal ( $\alpha=\alpha_{N}=0.8 \mathrm{GeV}^{-1}$ ) for the lowest, compact Fock states, the form factors can be explicitly calculated from the $N=3$ (valence) and $N=5$ (sea quark and gluon contributions) Fock state wave functions. These calculations can then be compared with the parameterisation of eq. 3.20 for all Fock states, with the parton distributions taken from 

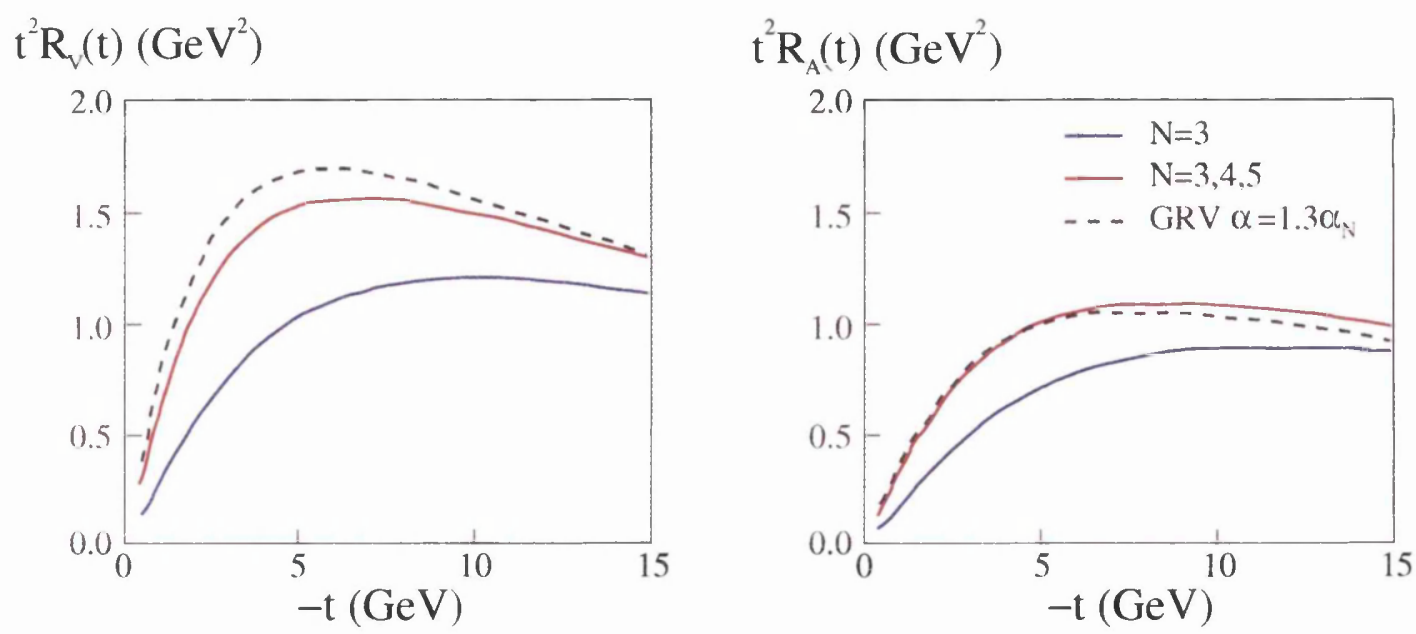

Figure 3.5: $t$-dependence of the Compton form factors $R_{V}$ and $R_{A}$ for all proton Fock states (dashed line) based upon eq. 3.20 and a fit to the GRV parton distributions $[46]$ with $\alpha=1.3 \alpha_{N}$. Also shown are models involving only the lowest proton Fock states $(N=3$, blue line and $N=5$, red line), which are based upon explicit wave function calculations with $\alpha=\alpha_{N}$.

recent DIS analyses (GRV) [46], with $\alpha=1.3 \alpha_{N}$. The results for the three cases, obtained in ref. [42], are shown in fig. 3.5. While the shape of the form factors does not significantly vary between the three models, the inclusion of the higher (non-valence) Fock states of the proton results in an appreciable increase in the overall magnitude of both $R_{V}$ and $R_{A}$.

The tensor form factor $R_{T}$, however, cannot easily be modelled using this technique, as both $R_{T}$ and the underlying GPD $E(x, 0 ; t)$ involve configurations where the individual parton helicities do not sum to the proton helicity. That is to say, they are naturally associated with configurations which have non-zero quark orbital angular momentum, and since very little is known about LCWF's of this type an alternative method is required. That the tensor form factor involves quark orbital angular momentum should be clear from the fact that the proton helicity-flip amplitude in eq. 3.14 is controlled by $R_{T}$, as well as from Ji's sum rule of eq. 2.14 .

The authors in ref. [37] have argued that an estimate of the magnitude of the tensor form factor is possible due to the close analogy between the Compton ratio $R_{T} / R_{V}$ and its electromagnetic equivalent $F_{2} / F_{1}$ at large $x$ and $-t$. This implies that the Compton form factor ratio should exhibit behaviour which is consistent with the recent observation $\left[33,34 \mid\right.$ that $F_{2} / F_{1}$ scales as $\Lambda / \sqrt{-t}$. This, in turn, has led to the introduction of the parameter $\kappa_{T}$ as a measure of the relative size 
of the tensor and vector form factors:

$$
\kappa_{T}=\frac{\sqrt{-t}}{2 m} \frac{R_{T}}{R_{V}} \simeq 0.37
$$

\subsubsection{Predictions}

To make predictions based upon the form factor estimates of eq. 3.20 and 3.21, one must transform the light-cone helicity amplitudes of eq. 3.13 and 3.14 to the more familiar CM helicity basis. This has been done in ref. [37], where, in addition, the hard subprocess helicity amplitudes of eq. 3.15 have been calculated at Nextto-Leading Order (NLO) in $\alpha_{s}$. The corresponding predictions for the differential cross section (again scaled by $s^{6}$ ) and the initial-state helicity correlations, $A_{L L}$ and $A_{L T}$, are shown in fig. 3.6. Agreement with the available cross section data is better than a factor of two, which is a considerable improvement on the best pQCD predictions. There is also an apparent mimicking of the scaling behaviour predicted by the constituent counting rules of eq. 3.4. This arises in the handbag description not through fundamental scaling arguments as before, but due to the broad maxima in the Compton form factors around $-t=8(\mathrm{GeV} / \mathrm{c})^{2}$ observed in fig. 3.5 .

The latest predictions for the spin observables at a photon energy of $5 \mathrm{GeV}$ are shown for calculations carried out with two different values of $\kappa_{T}$. The case $\kappa_{T}=0$ involves no proton helicity flip, whereas $\kappa_{T}=0.37$ corresponds to the estimate based upon the electromagnetic form factor ratio. The predictions for $A_{L L}$ in the handbag approach, which show that it is not particularly sensitive to proton helicity flip, differ notably from the pQCD predictions at large angles. $A_{L T}$, on the other hand, is far more sensitive to the choice of $\kappa_{T}$, and is predicted to be small in both cases. The presence of non-zero proton helicity flip introduces one further complication: the initial and final state spin observables may no longer be equated, as in the leading twist factorisation (eq. 3.9). Instead, the equivalence is only true to an accuracy dependent on the relative size of flip and non-flip amplitudes. Hence:

$$
\begin{aligned}
& K_{L L}=A_{L L}+\mathcal{O}\left(\kappa_{T}\right), \\
& K_{L T}=-A_{L T}+\mathcal{O}\left(\kappa_{T}\right),
\end{aligned}
$$

This introduces significant theoretical uncertainty in the predictions for the polarisation transfer components in the soft overlap framework. 

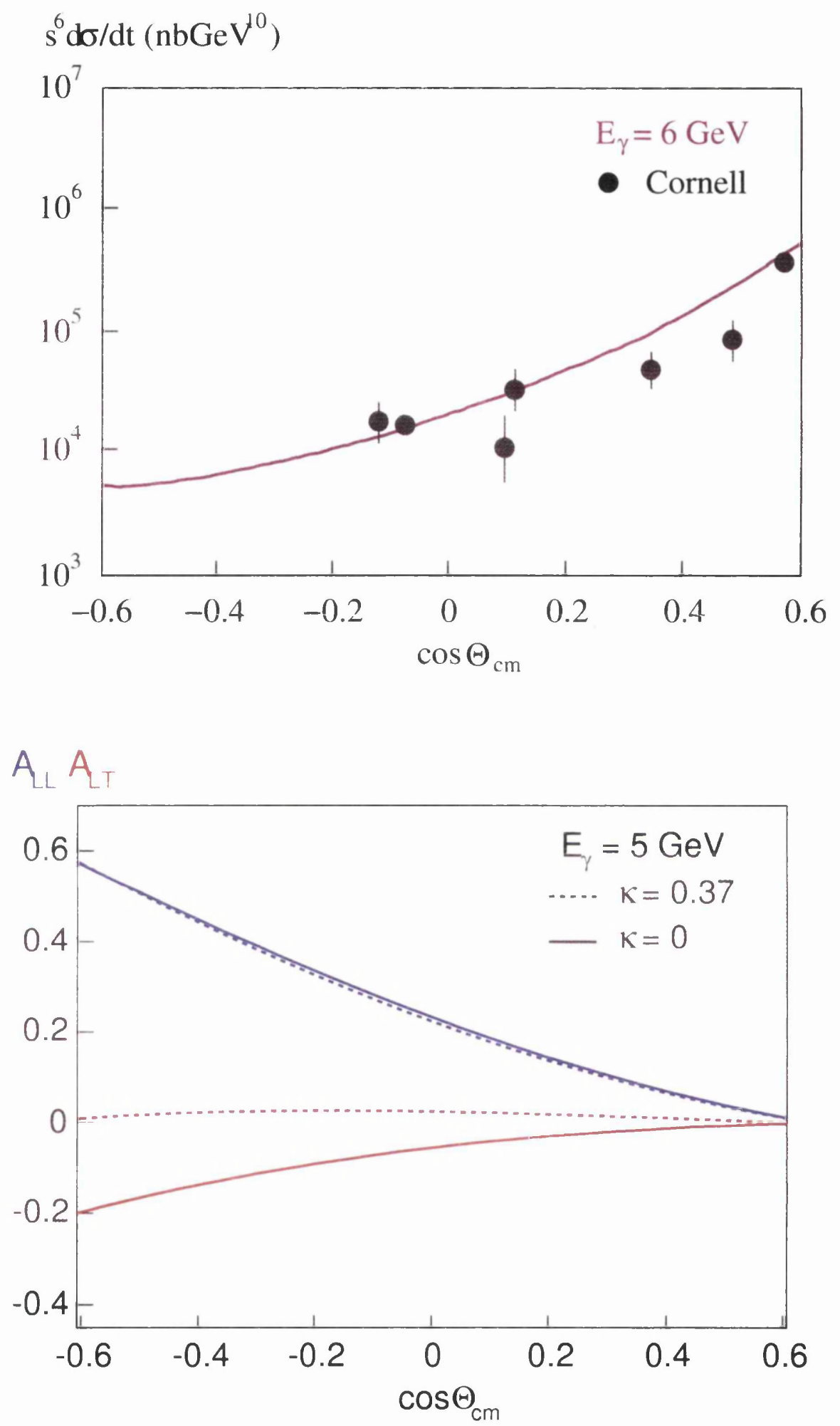

Figure 3.6: Latest soft overlap predictions [37,47]. Top: differential cross section, compared with Cornell data [30]. Bottom: spin observables $A_{L L}$ (blue) and $A_{L T}$ (red) for the cases, $\kappa_{T}=0.37$ (dashed) and $\kappa_{T}=0$ (solid). 


\subsection{Other Recent Calculations}

In this section we briefly highlight some other recent theoretical calculations for proton Compton scattering which further challenge some of the key assumptions that are inherent in the pQCD and soft overlap approaches. One feature is common to both these models: non-perturbative quarks and gluons are essential in successfully describing the scattering process.

\subsubsection{Relativistic Quark Models}

As discussed in sec. 3.3.2, the difficulty in treating the Compton tensor form factor is related to the problem of LCWF definitions in proton helicity-flip situations. This manifests itself most decisively with respect to the present measurement in the resulting relationship between the predicted initial-state observables and the measured polarisation transfer components (eq. 3.22). While this is the cause of some concern in the soft overlap approach, models of the proton have been formulated where the effects of quark orbital angular momentum are taken into account. Indeed, consideration of quark orbital angular momentum, as in Miller's Light-Front Cloudy Bag Model (LFCBM), is vital to the successful account of the JLab electromagnetic form factor data for the proton $[48,49]$.

There have been several incarnations of constituent quark models of the nucleon, starting with the M.I.T. bag model [50] in which the nucleon is modelled by three constituent quarks confined within a spherical potential. These constituent quarks are simply valence-quarks dressed by non-valence configurations of the nucleon, such as the sea-quarks and gluons, and therefore have a much larger effective mass. Constraints due to nucleon properties at low momentum transfer, where the dynamics is governed by effects such as spontaneous chiral symmetry breaking, led subsequently to the family of cloudy bag models [51], wherein extra degrees of freedom are added by including a pion field at the bag surface. Finally, the application to data at high momentum transfer naturally requires a relativistic treatment of the constituent quarks, which is achieved by performing the calculations in a Poincaré invariant framework using light-front dynamics.

The relativistic constituent quark calculations for Compton scattering [52] involve evaluating the handbag diagrams of fig. 3.4 in an impulse approximation. The resultant reaction amplitude depends on nucleon wave functions obtained from the LFCBM, neglecting the effects of the pion cloud which are unimportant 


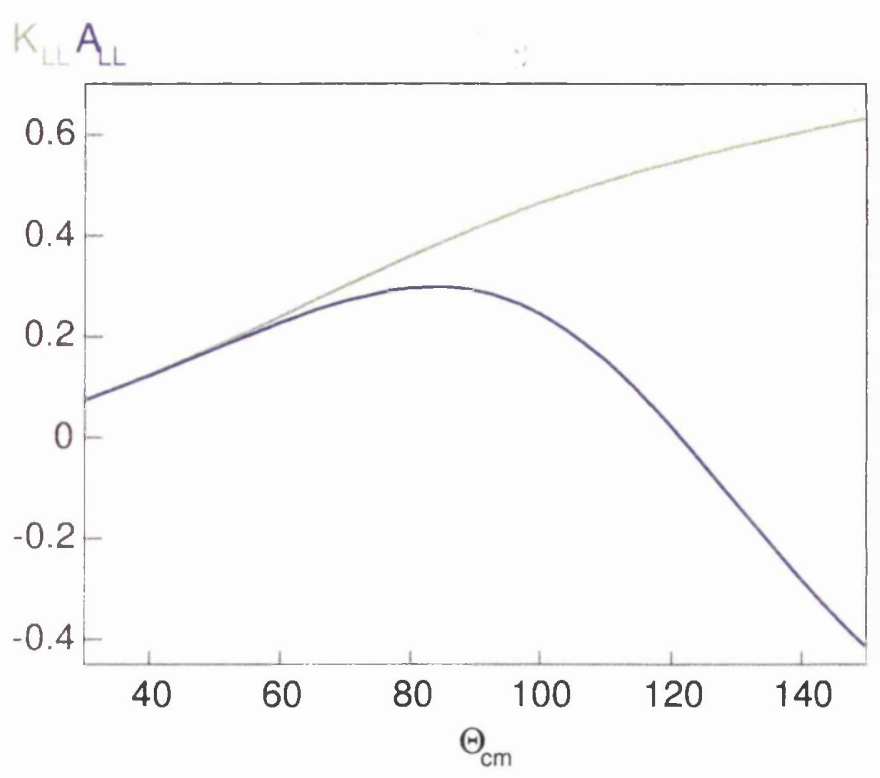

Figure 3.7: Predictions for the longitudinal spin observables $K_{L L}$ (green) and $A_{L L}$ (blue) from the latest LCFBM calculations [52, 53].

at high momentum transfer. The model parameters which give rise to these wave functions have previously been constrained by the JLab proton form factor data in the same kinematic regime [49]. Significant contributions to the wave functions from states involving non-zero quark transverse and orbital angular momenta arise precisely because of the relativistic nature of the calculations.

Reasonable agreement with the differential cross section data of ref. [30] has been obtained with minor modifications of the effective quark masses [52]. The same wave functions have also been used to make predictions for the proton spin observables defined in eq. 3.8, with the initial and final state observables calculated separately (unlike the previous two calculations). The longitudinal polarisation predictions can be seen in fig. 3.7: a large positive value has been predicted for the polarisation transfer, $K_{L L}$, similar to the soft-overlap prediction. Conversely, both transverse observables are expected to be zero in this model $\left(K_{L T}=A_{L T}=0\right)$. By far the most striking feature of the longitudinal predictions is the significant inequality between $K_{L L}$ and $A_{L L}$, which becomes more pronounced as one moves to larger backwards angles. The fact that $K_{L L} \neq A_{L L}$ is a direct consequence of proton helicity flip and, therefore, it is evidence for large contributions from states within the model involving non-zero quark orbital angular momentum. 


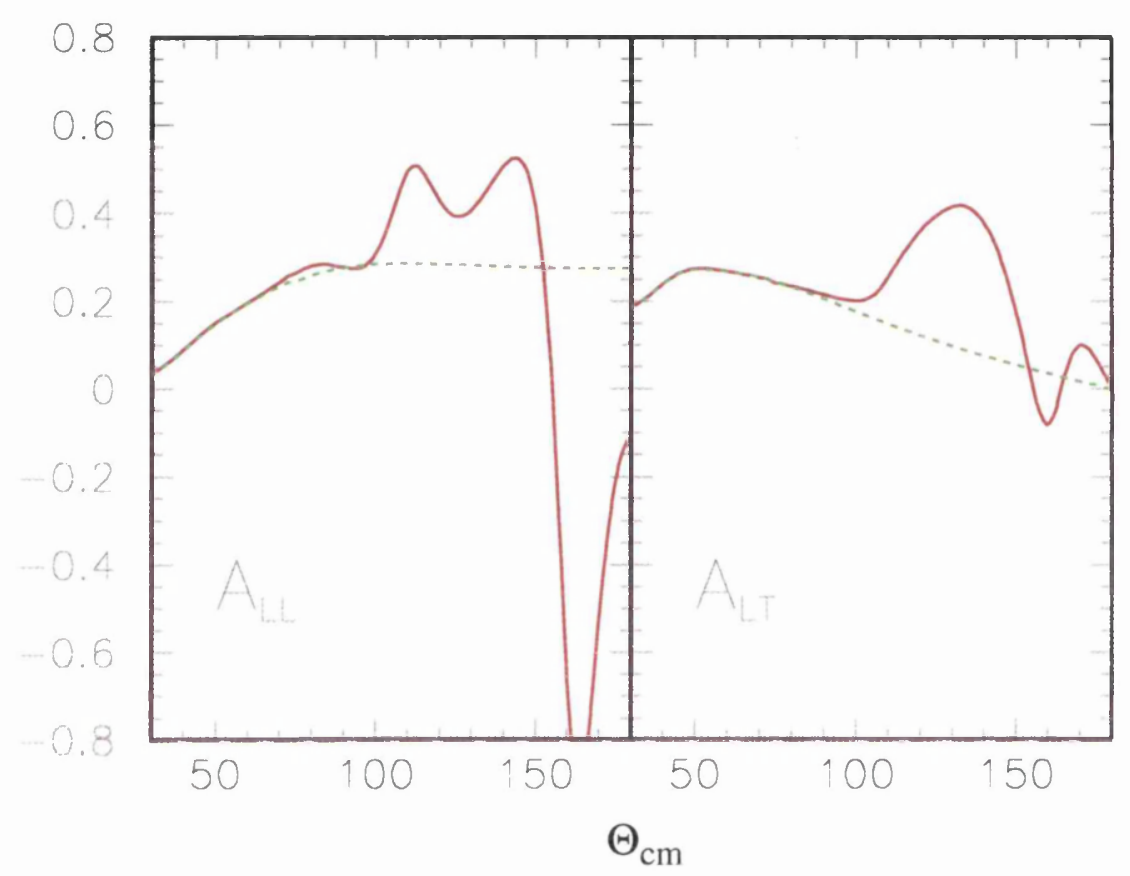

Figure 3.8: Predictions of the observables $A_{L L}$ (left) and $A_{L T}$ (right) from calculations based upon VMD and Regge exchange mechanism [55]. The full prediction (red) involves the contributions from both u-channel baryon exchange and twogluon exchange in the t-channel. The contribution from only the latter is shown in green.

\subsubsection{Regge Exchange Mechanism}

One assumption implicit in all previous calculations is the point-like photonparton $(\gamma q)$ coupling in the hard scattering subprocess. At presently accessible photon energies, however, this assumption becomes rather tenuous due to the fact that the photon is not the clean, purely electromagnetic probe that has been assumed thus far. Within the constraints of the uncertainty principle it is possible for the photon to fluctuate into quark/anti-quark pairs, resulting in an interaction which will then resemble a purely hadronic process. This fluctuation and the fact that the photon has the quantum numbers of a vector meson $(\rho, \phi$ and $\omega)$ is the basis of Vector Meson Dominance (VMD) [54]. Indeed, the lifetime of the hadronic component of a photon at $4 \mathrm{GeV}$ corresponds to a fluctuation into a $\rho$ meson over a distance scale of around three times the size of the nucleon - very far from a point-like coupling. 
Working with vector meson photo-production data, Cano and Laget $[55,56]$ have achieved reasonable success using a mechanism based upon a Regge description of the hadronic interaction between vector meson and proton. The Regge model of strong interactions was the predecessor of QCD, wherein the amplitude of a particular process can be viewed as an exchange of Regge trajectories, which represent the superposition of all possible particles that can be exchanged [57]. In the case of vector meson production at moderate momentum transfer, it turns out that the data is well described by interactions and exchanges involving constituent quarks and gluons. In this model, two non-perturbative (or dressed) gluons are exchanged in the t-channel and interact with two different quarks within the proton. This process alone does not satisfactorily describe the data over the full kinematic range; instead, it is necessary to include Regge trajectories based upon t-channel meson exchange at forward angles, and u-channel baryon exchange at backwards angles.

The same approach can be applied to proton Compton scattering, where the outgoing photon can be formed in a similar interaction through an intermediate vector meson state. No additional model parameters are required to relate the photon and meson production amplitudes. All that is necessary to combine the two data-sets is a normalisation of the Compton observables involving the radiative decay constant of the $\rho$ meson. Note that the predictions obtained from this model are based upon adjusting the parameters to fit the available data. As a result, good agreement has been achieved for the differential cross section data of both vector meson production and Compton scattering. The predictions which have been calculated using the same model parameters for the Compton spin observables, $A_{L L}$ and $A_{L T}$, are shown in fig. 3.8 .

\subsection{Summary}

From a general discussion in the previous chapter on the progress made in understanding the nucleon, through the introduction of hard exclusive processes and the GPD's which are associated with them, we have tried to place the present measurement of polarisation transfer in proton Compton scattering in the appropriate context. It should be clear from the discussion in this chapter that the paucity of Compton scattering data in the high momentum regime is not reflected by the intense theoretical activity in recent years. Indeed, four distinct theoretical approaches have been discussed, which involve two fundamental reac- 


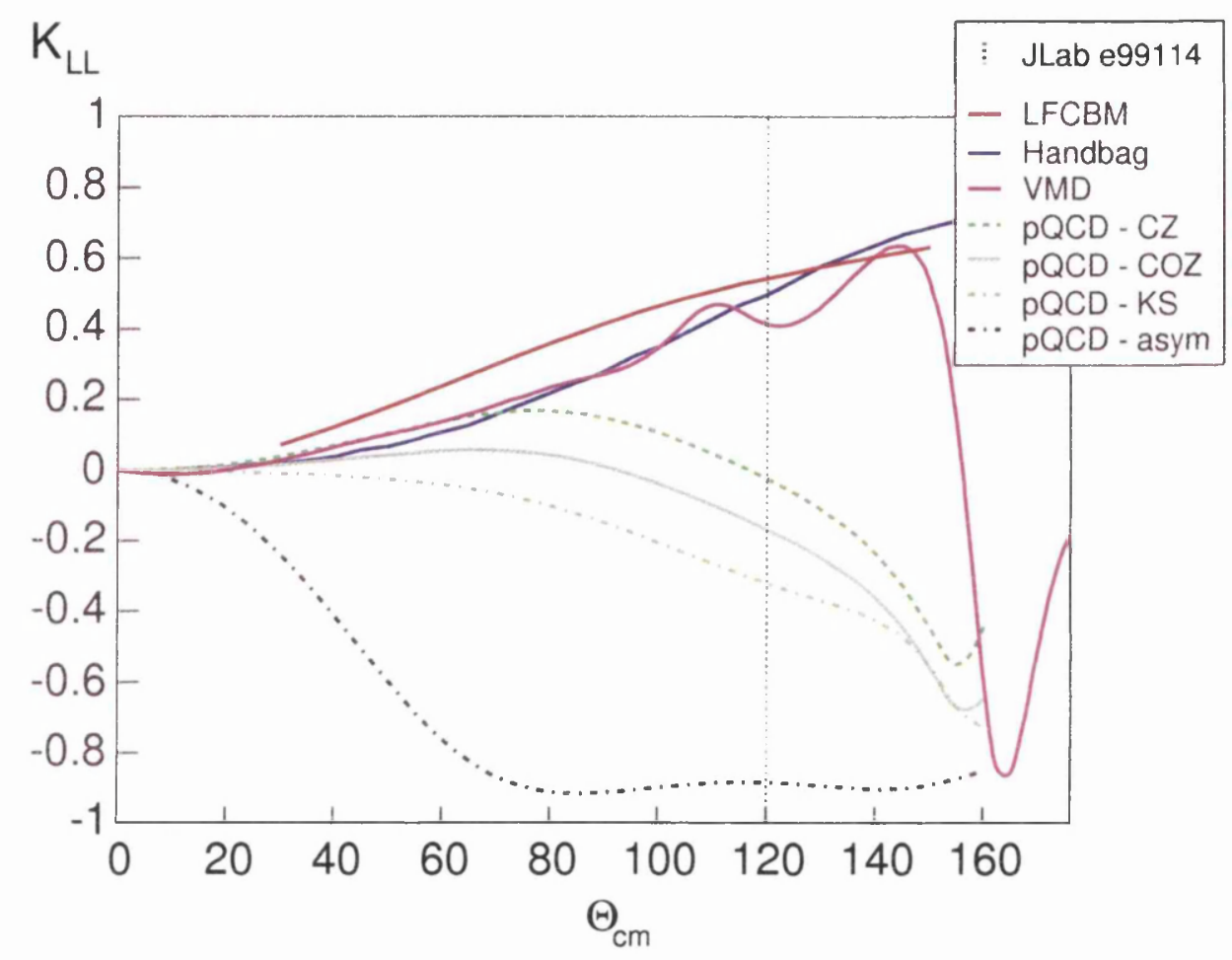

Figure 3.9: All currently available predictions for polarisation transfer $K_{L L}$ from $p Q C D$ (green), soft overlap (blue), LFCBM (red) and Regge exchange (magenta) calcultions. The vertical dotted line shows the value of $\Theta_{c m}$ at which the present measurement took place.

tion mechanisms relating to the means by which the momentum is transferred to the proton as a whole:

1. The leading twist pQCD factorisation, where the momentum is shared amongst three valence-quarks via two hard gluon exchanges.

2. The handbag factorisation, which involves distribution of the momentum from a single active quark to the rest of the proton through overlaps of soft light-cone wave functions.

While there is widespread acceptance that the transition to the purely perturbative regime is likely to occur at larger momentum transfers than presently available at Jefferson Lab, there is, as yet no definitive evidence to distinguish which of these two mechanisms, if any, is dominant in the few $\mathrm{GeV}$ range.

The various predictions for the longitudinal polarisation transfer component $K_{L L}$ are shown in fig. 3.9 ; the contrast between the pQCD and handbag pre- 
dictions at large scattering angles could hardly be more pronounced. For this reason, a measurement of $K_{L L}$ for an incident photon energy of $3.2 \mathrm{GeV}$ and a scattering angle of $\Theta_{c m}=120^{\circ}$ has taken place in Hall A at Jefferson Lab. Alongside the question of the dominant reaction mechanism, Compton scattering has the potential to reveal a great deal more about the non-perturbative structure of the proton, with the Compton form factors of eq. 3.16 allowing access to the underlying GPD's. These, in turn, provide complementary information to the already available electromagnetic form factors and DIS parton distributions. The real Compton scattering program at Jefferson Lab is described in the following chapter and, in addition to the polarisation transfer, involved precise measurement of the differential cross section. It is believed that the combined data from this experiment will ultimately provide a model-independent determination of all three Compton form factors. 


\section{Chapter 4}

\section{Experimental Apparatus}

The Jefferson Laboratory Hall A experiment E99-114, Real Compton Scattering (RCS) from the proton [58], ran from January to March 2002. The main aims of the experiment were:

1. To measure the differential cross section for the $p\left(\gamma, \gamma^{\prime} p\right)$ reaction over a broad range of scattering angles.

2. To determine the recoil proton polarisation transfer observables at a single kinematic point.

The experimental technique, shown schematically in fig. 4.1, is common to both types of measurement. Specifically for the latter case, a continuous-wave electron beam with energy $3.47 \mathrm{GeV}$ and current $\leq 40 \mu \mathrm{A}$ was incident on a $6 \%$ copper radiator, producing a mixed beam of electrons and bremsstrahlung photons, which were then scattered from a $15 \mathrm{~cm}$ liquid hydrogen target. For incident photons near the bremsstrahlung endpoint (with an average photon energy of $3.22 \mathrm{GeV}$ ), a final state proton and photon were detected in coincidence using a magnetic spectrometer and photon calorimeter, respectively. The magnetic spectrometer, located at a laboratory angle of $20^{\circ}$, is one of the pair of standard Hall A high resolution spectrometers and was equipped with a focal plane polarimeter. Scattered photons with a mean laboratory angle of $65^{\circ}\left(\Theta_{c m}=120^{\circ}\right)$ were detected in a highly-segmented electromagnetic calorimeter built specifically for this experiment. The combined angular precision of the two detectors had to be exceptional to distinguish between the Compton photons and those due to the dominant $p\left(\gamma, \pi^{0} p\right)$ background reaction, while a magnet between target and calorimeter was required to identify and reject the kinematically indistinguishable $p\left(e, e^{\prime} p\right)$ events. 


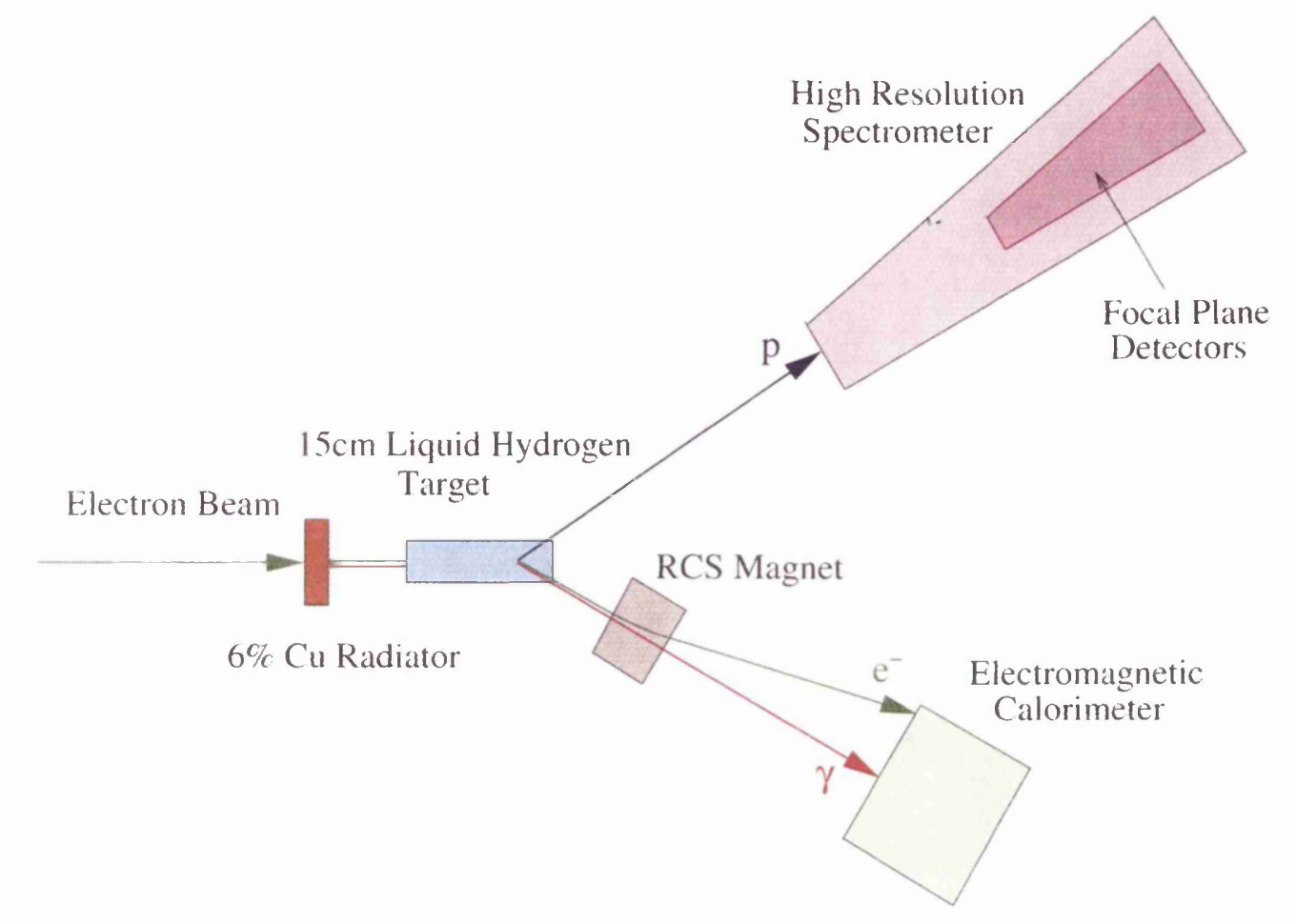

Figure 4.1: Schematic of the experimental layout for E99-114 in Hall A at JLab.

\subsection{The Thomas Jefferson National Accelerator Facility}

In the mid 1980's, it was becoming clear that a dedicated laboratory to study hadronic structure and nuclear properties at the sub-nucleonic level, at energies of a few $\mathrm{GeV}$, was of primary importance to the subatomic physics community. Design and commissioning of such a facility was undertaken jointly by the U.S. Department of Energy and the Southeastern Universities Research Association (SURA). Scientific data were first taken in 1995 at the Thomas Jefferson National Accelerator Facility (Jefferson Lab or Jlab) in Newport News, Virginia. It has since matured to become the world's premier facility for studying the transition from baryon-meson degrees of freedom to the more fundamental quark-gluon picture.

The Continuous Electron Beam Accelerator Facility (CEBAF) at JLab [59] is a unique tool, designed and built to deliver a high quality, continuous-wave electron beam to three experimental halls simultaneously. A diagram of the machine is shown in fig. 4.2. Electrons are injected at a few $\mathrm{MeV}$ into the main 
accelerator consisting of two anti-parallel linear accelerators (or linacs), linked by nine recirculation beam lines in a race-track configuration. Recirculation of the beam is possible up to five times for maximum beam energy, or it can be extracted after each complete pass and delivered to one or more of the experimental halls $\mathrm{A}, \mathrm{B}$ and $\mathrm{C}$.

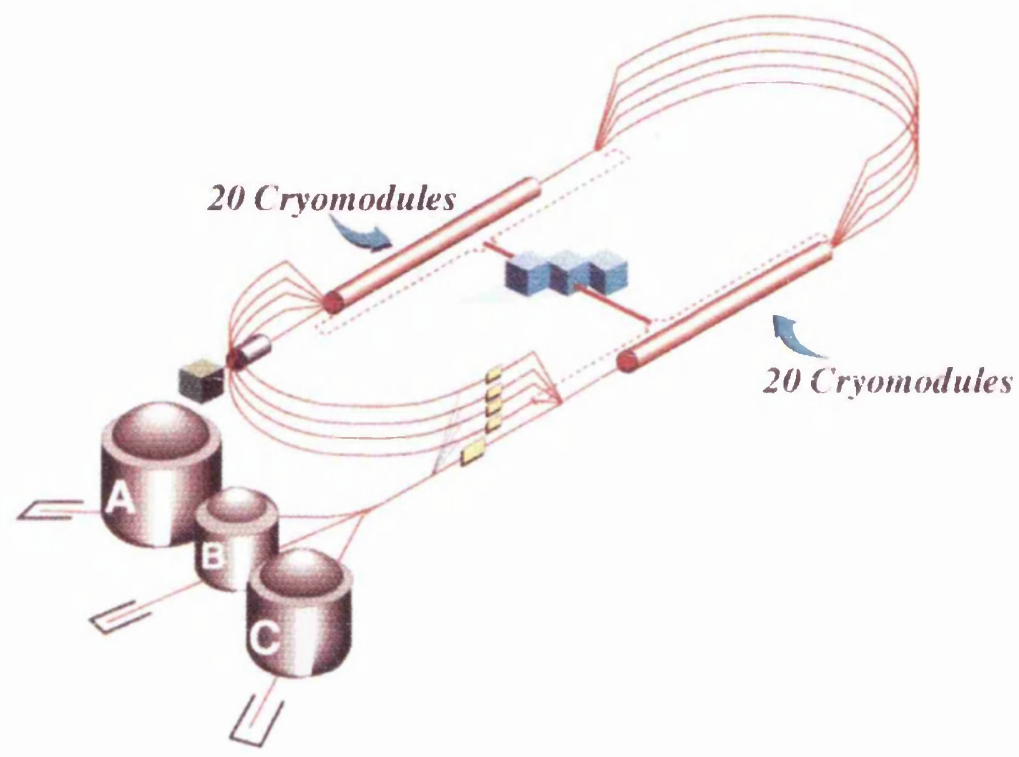

Figure 4.2: The CEBAF machine at Jlab: a race-track configuration with 20 cryomodules per linac and nine recirculation arcs.

At the time of construction, CEBAF was the largest superconducting radio frequency (s.r.f.) project in the world. Each linac consists of 20 cryomodules, each of which contain four Niobium s.r.f. cavities cooled by hundreds of litres of liquid helium at $2 \mathrm{~K}$. The major advantage of superconducting technology over more traditional means is that much less r.f. power is needed, as no heat is produced by ohmic processes within the cavities. Thus the machine may be operated continuously, as opposed to the pulsed operation necessary in a room temperature linac to avoid meltdown. The magnetic optics that form the recirculation arcs and beam transport sections to the halls consist of a myriad of dipole, quadrupole, sextapole and septum elements. The original design goals for an acceleration of $0.4 \mathrm{GeV}$ per linac have been surpassed, and CEBAF is now capable of delivering beam energies ranging from a few hundred $\mathrm{MeV}$ to $5.7 \mathrm{GeV}$, at currents of up to $120 \mu \mathrm{A}$ in Halls $\mathrm{A}$ and $\mathrm{C}$ and $\sim 1 n A$ in Hall $\mathrm{B}$, where detection systems limit 
luminosity.

The polarised electron beam gives access to a wealth of spin observables. It is produced by light from three $499 \mathrm{MHz}$ r.f. gain-switch diode lasers, incident on a strained gallium arsenide (GaAs) photocathode under high vacuum. These lasers produce three interlaced beams at the source, spaced by $120^{\circ}$ of r.f. phase and accelerated in a common $1497 \mathrm{MHz}$ field. The use of r.f. separators at the hall entrances allows beam buckets, each with different charge and polarisation states, to be delivered to the three halls simultaneously. Two electro-optical devices called Pockels cells, one used as a quarter-wave plate and the other as a half-wave plate, are used to produce circularly polarised laser light, which causes photo-emission of longitudinally polarised electrons from the GaAs. The degree of polarisation at the halls can be up to $85 \%$, and is compensated for spin precession in the beam transport systems by varying a Wien filter (spin rotator) at the source. Adjusting the voltage on the quarter-wave Pockels cell, pseudo-randomly at a rate of $30 \mathrm{~Hz}$, results in a corresponding flip in the longitudinal direction of the electron spin so that it is either parallel or anti-parallel to the direction of propagation (i.e. \pm beam helicity).

\subsection{Experimental Hall A}

Hall $\mathrm{A}$ is the largest of the three experimental halls at Jefferson Lab. It contains two optically identical High Resolution Spectrometers (HRS) located on either side of the electron beamline, which can be rotated about the target position over a wide range of angles. Between the beam switch-yard and the target (which sits at the centre of the hall) there are various devices to monitor and measure beam conditions. A much fuller description of the standard apparatus in the hall can be found in ref. [60].

\subsubsection{Beamline Apparatus}

Electron beam parameters are measured by several devices situated along the beamline, upstream of the target, near the entrance to the hall. Measurements of position, current, energy and longitudinal polarisation are possible, with some intentional redundancy to allow cross checking. Some typical beam parameter values for the present experiment and the uncertainties associated with their measurement [61] are given in table 4.1. 


\begin{tabular}{|l|c|c|c|}
\hline Beam Parameter & $\begin{array}{c}\text { Beamline } \\
\text { Device(s) }\end{array}$ & $\begin{array}{c}\text { Measured } \\
\text { Value }\end{array}$ & Accuracy (absolute) \\
\hline \hline Position (at target) & BPM/Harp & - & $140 \mu m$ \\
Direction (at target) & BPM/Harp & - & $30 \mu r$ \\
\hline Current & BCM & $40 \mu \mathrm{A}$ & $\leq 2 \times 10^{-3}$ \\
\hline Energy & ARC/ep & $3.471 \mathrm{GeV}$ & $2 \times 10^{-4}$ \\
\hline Polarisation & Møller & 0.766 & 0.026 \\
\hline
\end{tabular}

Table 4.1: Typical values measured for the various electron beam properties described in the text, together with the associated uncertainties.

Two Beam Position Monitors (BPM), located about $7 m$ and $1 m$ upstream of the target, provide information on the position and direction of the beam, both on an event-by-event and time averaged basis. A BPM consists of four antennae surrounding the beamline in a diamond configuration. As it passes, the electron beam induces a signal in the antennae which can be measured and used to accurately reconstruct its position. The BPM's are complemented by the presence of several wire (harp) scanners in the beamline, which are regularly surveyed with respect to absolute Hall A coordinates and used to cross-calibrate the BPM devices.

Two cylindrical resonant cavities, tuned to the radio frequency of the electron beam and situated about $24 m$ upstream of the target, form the Hall A Beam Current Monitors (BCM). A coaxial loop antenna inside each cavity detects the resonant magnetic field produced when a beam bucket passes. The effective charge in the bunch is proportional to the size of the field and thus to the signal produced in the antenna. This signal is then recorded using a voltage-to-frequency converter and scaler.

To measure the absolute value of the energy of the electrons in the beam, two distinct methods are employed. Firstly, eight dipole magnets between the beam switch-yard and the hall entrance deflect the electrons through a nominal angle of $34.3^{\circ}$. Wire scanners before and after the magnets accurately determine this angle, which along with the measured arc field integral $\int \vec{B}$. $\overrightarrow{d l}$, is used to calculate the electron momentum. The second technique utilises the $p\left(e, e^{\prime} p\right)$ reaction and a purpose built silicon strip detector located $17 \mathrm{~m}$ from the centre of the hall. A polyethylene $\left(\mathrm{CH}_{2}\right)$ target is placed in the path of the beam and the scattered electron and recoil proton tracks are measured in the strip detector. From a straightforward analysis of the kinematics of this reaction the incoming 
electron energy can be reconstructed.

Finally, measurements of physical polarisation observables depend critically upon accurately determining the degree of polarisation of the incident electron beam. An intrusive way to measure this is by using the Møller polarimeter. This device makes use of the asymmetry in Møller scattering of polarised beam electrons from atomic electrons in a magnetized target foil. Both are detected in coincidence by the lead-glass counters of a small magnetic spectrometer, consisting of three quadrupoles and a dipole magnet [62]. The asymmetry in the scattering cross section due to the longitudinal component of the beam polarisation, along with the foil target magnetisation, are then used to compute the beam polarisation. The relative systematic uncertainty associated with such a measurement is around $3 \%$, which is mainly attributable to the uncertainty in the target magnetisation. A single measurement of this type was made during the present experimental program, the results of which together with a discussion on the stability of the beam polarisation can be found in Appendix B.

\subsubsection{Cryogenic Target System}

The Hall A cryogenic target system [63] consists of three independent fluid transfer loops: liquid hydrogen $\left(L H_{2}\right)$, liquid deuterium $\left(L D_{2}\right)$ and gaseous helium. Two liquid hydrogen targets were used in this experiment, one of which had a bremsstrahlung radiator attached.

The hydrogen target cells, together with a selection of dummy and solid targets, are mounted on a vertical ladder inside the scattering vacuum chamber which can be moved remotely. The chamber has entrance and exit ports for the beam and $0.38 \mathrm{~mm}$-thick aluminium windows spanning the full angular range covered by the spectrometers $\left(12.5^{\circ}<\Theta_{H R S}<165^{\circ}\right)$. Target gas is supplied at room temperature and cooled by liquid helium at $15 K$. Coolant supply is regulated by a separate Joule-Thomson (JT) valve for each loop. A high power heater and circulating fans operate on a feedback system to maintain a steady temperature and pressure as the beam current, and therefore the heat load on the target, varies. With the high beam currents available in Hall $\mathrm{A}$, the small beam diameter, and hence the highly localised heat deposition, are capable of causing local damage to the cell. To prevent this happening, the beam is rastered (scanned back and forth at frequencies of $17-24 \mathrm{kHz}$ ) in the horizontal and vertical directions by two dipole magnets $17 m$ upstream of the target. 
The liquid hydrogen targets themselves are contained within cylindrical aluminium cells, $15 \mathrm{~cm}$ long and $63.5 \mathrm{~mm}$ in diameter. The thicknesses of the upstream window, downstream window and side wall of the cells are $71 \mu \mathrm{m}, 102 \mu \mathrm{m}$ and $178 \mu \mathrm{m}$, respectively. The operational temperature is $19 \mathrm{~K}$ and the pressure is $0.17 \mathrm{MPa}$, corresponding to an average density of $0.0723 \mathrm{gcm}^{-3}$. The bremsstrahlung radiator, attached directly upstream of one of the target cells, consists of a set of copper foils of total thickness $0.81 \mathrm{gcm}^{-2}$, which is equivalent to $\sim 6 \%$ of one radiation length.

\section{Mixed $e^{-} / \gamma$ beam}

Coincident measurements involving processes at high momentum transfer are characterised by low cross sections and therefore require very high luminosity and moderately large acceptance detectors to achieve reasonable statistical accuracy. In the present case, the means of achieving a sufficiently high incident photon flux was to use bremsstrahlung produced in the $\mathrm{Cu}$ radiator. This necessarily meant that there was a mixed electron/photon beam on target, as no means were available to sweep away the electron beam.

When a light charged particle, such as an electron, interacts with the electric field of an atomic nucleus, it is accelerated by the strong localised field and produces radiation in the form of real photons. This process is known as braking radiation or bremsstrahlung. It is the dominant process by which high energy electrons interact with matter and lose energy. The energy loss is proportional to $Z^{2} E / m^{2}$, where $Z$ is the atomic number of the radiator material and $E$ and $m$ are the energy and mass of the electron. An essential feature of the bremsstrahlung process with regard to the present measurement is the fact that the polarisation of the electron is almost completely transferred to the photon (see Appendix B).

The energy distributions of photons and post-bremsstrahlung electrons produced by a monochromatic electron beam incident on a copper radiator [64] are shown in fig. 4.3. The energy of the incoming electrons define the bremsstrahlung endpoint energy. It is clear from this figure that the ratio of electrons to photons at the endpoint is at a maximum value (if, as in the present case, the probability of multiple processes is low). Because the process under investigation involves elastic scattering kinematics, the position and acceptance of the HRS and calorimeter in the hall uniquely define an incident particle energy range $\left(E_{\max }-E_{\min }\right)$. For production data-taking periods the detector position, and hence this range, was 


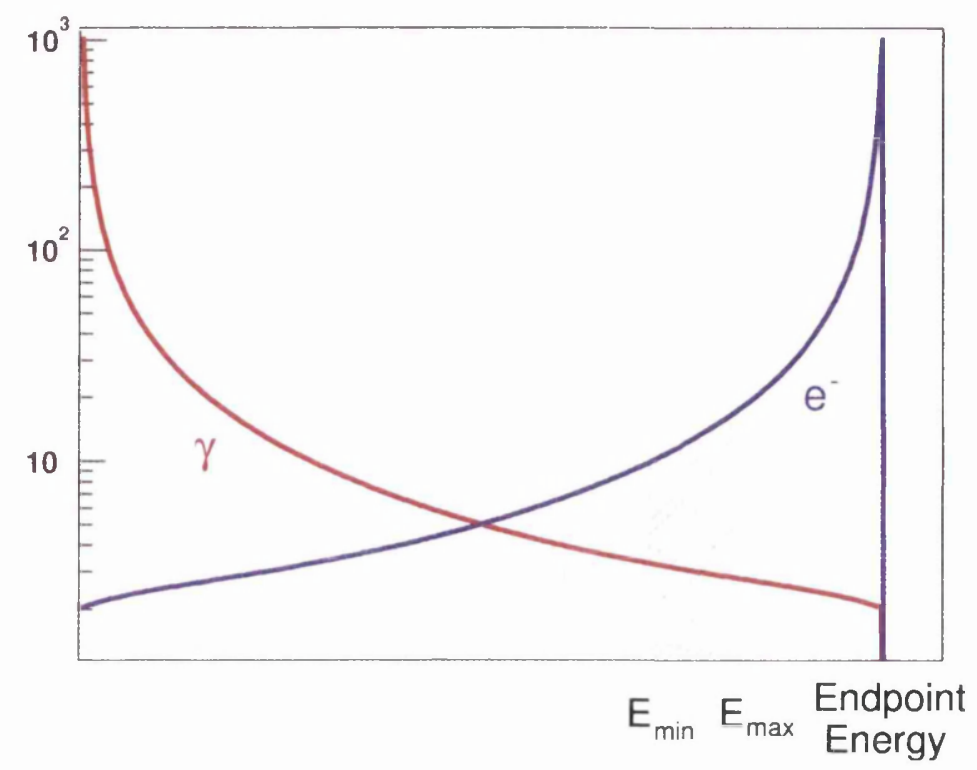

Figure 4.3: A typical bremsstrahlung energy spectrum for photons (red) and electrons (blue). The energy of the electron beam is labelled as the endpoint energy; the range $E_{\min } \rightarrow E_{\max }$ is determined by the position and acceptance of the detectors.

chosen to be significantly below the endpoint energy ${ }^{1}$ (the so-called off-endpoint kinematics). This choice was motivated by the need to reduce the number of background elastic electron-proton $(e p)$ scattering events within the acceptance of the detectors, although a balance had to be reached between avoiding the endpoint electrons and ensuring there was a sufficiently large flux of high energy photons.

\subsubsection{High Resolution Spectrometer}

Both HRS devices were designed to have moderately large acceptance in both angle and momentum, acceptance for extended targets and very high resolution of momentum, in-plane angle and position. In the current experiment, the spectrometer situated on the left-hand side of the beamline (with respect to incoming beam direction) was used to detect the recoil proton. The other spectrometer was rotated to a large backward angle to make way for the photon calorimeter and the accompanying electronics and cables.

The magnetic elements of the HRS consist of three superconducting $\cos 2 \theta$

\footnotetext{
${ }^{1}$ It is worth noting that $E_{\max }$ was chosen to be around $100 \mathrm{MeV}$ below the endpoint, which is exaggerated in fig. 4.3.
} 


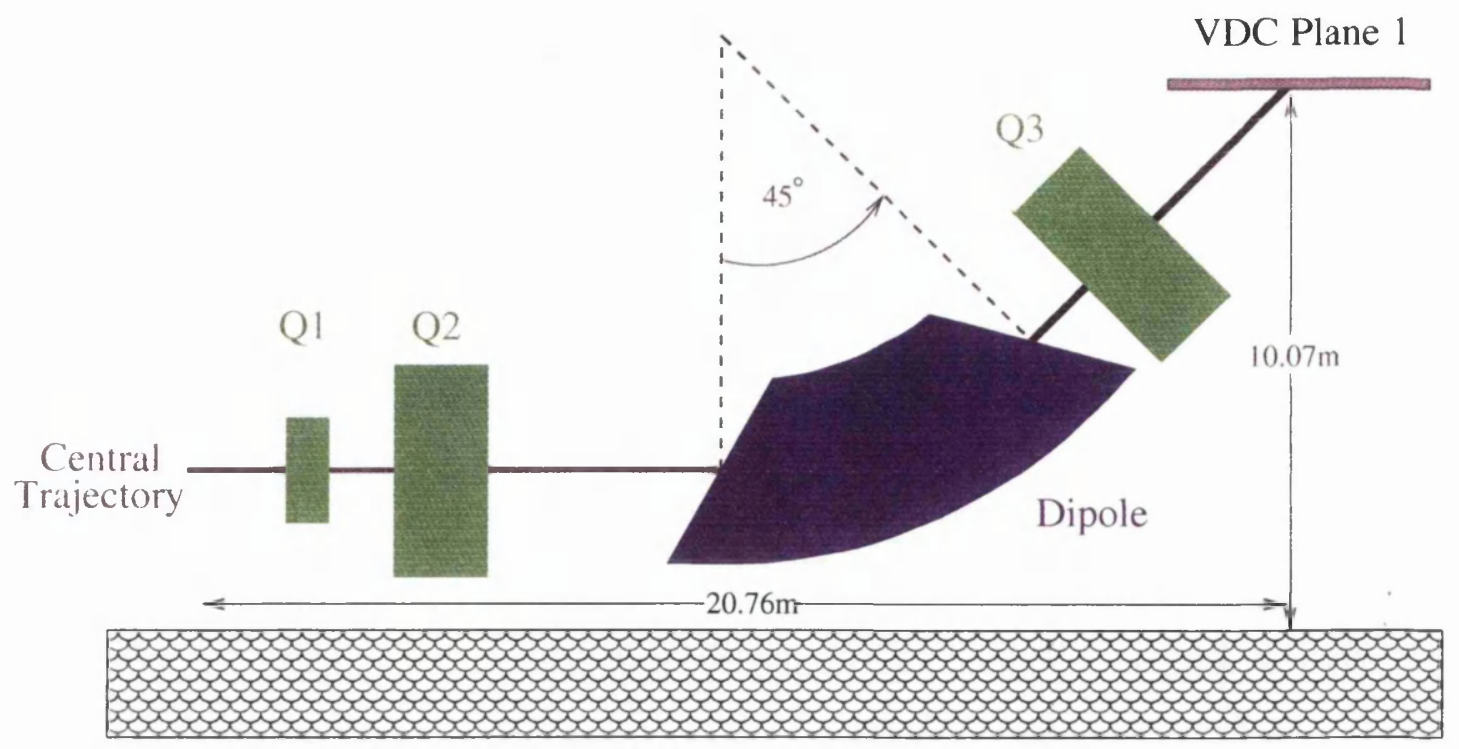

Figure 4.4: A side view showing the magnetic layout of the HRS. All magnet dimensions represent the effective field boundaries.

quadrupoles and a superconducting indexed dipole, in a $Q Q D_{n} Q$ arrangement (fig. 4.4). The dipole has a vertical bend of $45^{\circ}$ which decouples, in first order, a measurement of momentum from a measurement of target position. It provides focusing in the vertical plane by a non-uniform radial field component due to its trapezoidal cross section. Quadrupole $Q_{1}$ also focuses in the vertical plane, whereas $Q_{2}$ and $Q_{3}$ both provide vertical defocusing. All four magnets are refrigerated by liquid helium at a temperature of $4.5 \mathrm{~K}$. For a given value of central momentum the field values in each magnet are set remotely using automated control software. The fields are measured and monitored by Nuclear Magnetic Resonance (NMR) probes in the dipole and Hall probes in the quadrupoles. The magnetic characteristics of the HRS-left are shown in table 4.2.

\begin{tabular}{|ll|c|}
\hline \multicolumn{2}{|l|}{ Momentum Resolution } & $2 \times 10^{-4}$ \\
\hline Momentum Range & $0.3-4.3 \mathrm{GeV} / \mathrm{c}$ \\
\hline Momentum Acceptance & $\pm 4.5 \%$ \\
\hline Angular Acceptance & Horizontal & $\pm 28 \mathrm{mr}$ \\
& Vertical & $\pm 60 \mathrm{mr}$ \\
\hline Angular Resolution & Horizontal & $0.6 \mathrm{mr}$ \\
& Vertical & $2.0 \mathrm{mr}$ \\
\hline
\end{tabular}

Table 4.2: Spectrometer characteristics of the HRS-left. 


\subsubsection{HRS Focal Plane Detectors}

The detector pacakage is housed within a shielding hut and is located on top of the HRS structure, immediately after $Q_{3}$, at the spectrometer focal plane. The flexibility of the HRS detector configuration means it can be tailored to the needs of individual experiments. For the present experiment, an extended version of the standard hadron focal plane stack was used on the left-arm (fig. 4.5). The detector package was composed of:

- Two Vertical Drift Chambers (VDC) for precise tracking of the proton trajectories.

- A Focal Plane Polarimeter (FPP) for measuring recoil proton polarisation. It consists of two sets of straw chambers and two analyser blocks, and is described below in sec. 4.3 .

- Three scintillator planes for trigger and time-of-flight information. These detectors are discussed in relation to the experimental trigger in sec. 4.5.1.

- An aerogel threshold Cerenkov and a lead-glass shower counter, both for particle identification. Neither of which were used in the polarised part of the measurement.

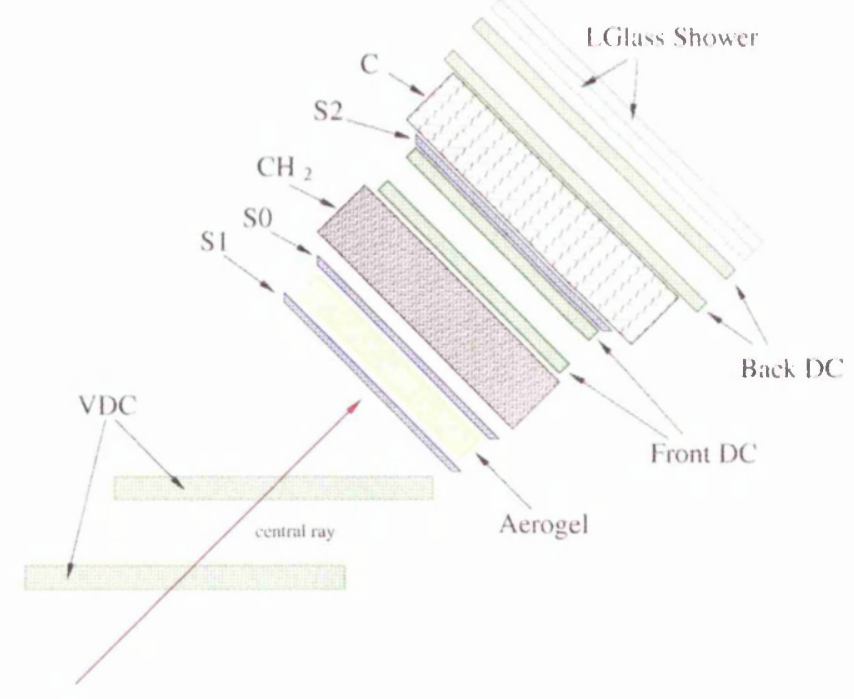

Figure 4.5: Diagram of the HRS-left detector package used during the current measurement. The individual components are described in the text. 


\section{Vertical Drift Chambers}

The VDC's [65] are the most crucial piece of the Hall A detector arsenal. Combined with knowledge of the optical transfer properties of the spectrometer, the VDC hit coordinates are used to reconstruct the proton trajectory, reaction vertex and momentum at the target (this procedure is discussed in sec. 5.2). Located between the third quadrupole and all other detectors, they are mounted parallel to the hall floor on two rails attached to the spectrometer support structure, and aligned to an accuracy of around $100 \mu \mathrm{m}$. Physically, the chambers are approximately $240 \mathrm{~cm}$ long, $40 \mathrm{~cm}$ wide and $10 \mathrm{~cm}$ high, covering an active area of $211.8 \times 28.8 \mathrm{~cm}^{2}$. Their relative orientation is shown in fig. 4.6. Each chamber contains two wire planes ( $u$ and $v$ ) with a total of 368 gold-plated tungsten wires per plane, orientated at $+45^{\circ}$ and $-45^{\circ}$ relative to the central trajectory. Goldplated Mylar cathode planes are located between the wire planes and operated at a high voltage of $-4.0 \mathrm{kV}$. A mixture of $62 \%$ argon and $38 \%$ ethane flow through the chambers at a rate of 5 litres per hour.
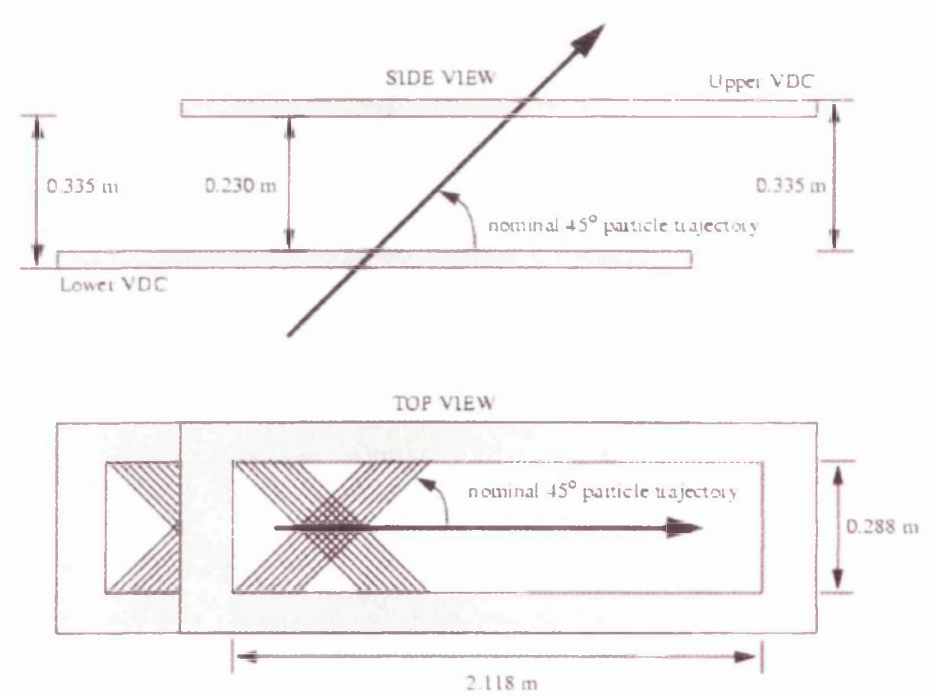

Figure 4.6: The postion of the VDC's relative to each other, and to the nominal central trajectory.

When a charged particle passes through one of the chambers, it ionises the gas atoms and produces a trail of electrons and ions. Far from a sense wire, where the electric field between wire and cathode is uniform and parallel, electrons drift toward the wire with a constant velocity. As they get closer, the field has a stronger radial nature, causing them to accelerate and produce a secondary 
electron avalanche. This avalanche generates a negative pulse on the wire which is processed by a pre-amplifier/discriminator card. The logic output of each discriminator is sent to a multi-hit (LeCroy Fastbus 1877) Time-to-Digital Converter (TDC). The measured drift time and known electron drift velocity can then be used to calculate the perpendicular distance between wire and particle track. A typical track generates signals in about five wires per plane. The position resolution at the focal plane is approximately $100 \mu \mathrm{m}$ and the angular resolution is $\sim 0.5 \mathrm{mr}$.

\subsection{Focal Plane Polarimeter}

Extracting information on the recoil proton polarisation requires a measurement of the polarisation components at the focal plane, coupled with an understanding of the nature of spin precession in the spectrometer magnetic fields. The Hall A device for measuring the focal plane proton polarisation, by means of secondary nucleon scattering in an analyser, is the FPP [66].

\subsubsection{Recoil Proton Polarimetry}

When a proton scatters from another nucleon, a spin-orbit coupling in the strong interaction between the two results in a sensitivity to the direction of the incoming proton spin. For a sample of incoming protons with their spins preferentially aligned in a given direction, this gives rise to an azimuthal asymmetry in the observed direction of scattering. The sample, however, will be strongly contaminated by events where the proton scatters via the electromagnetic interaction in the analyser, often referred to as Coulomb or multiple scattering. These events differ from those of interest because they exhibit no explicit dependence on the initial state of the proton spin and are characterised by a narrower spread $\left(<5^{\circ}\right)$ in polar scattering angle. The technique of utilising secondary scattering to extract polarisation observables is in general use for protons $[67,68]$ as well as for neutrons [69]. The common features of these polarimeters are tracking devices before and after an analyser, to precisely measure and reconstruct events which scatter in the analyser material.

The Hall A FPP has been used in numerous experiments to extract meaningful data on proton polarisation at the spectrometer focal plane. In its standard configuration it consists of two sets of straw-tube drift chambers, which measure 
proton trajectories before and after the protons pass a $51 \mathrm{~cm}$-thick carbon analyser block. For the first time, however, a new dual analyser configuration of the FPP was used during the present measurement, with a $44 \mathrm{~cm}$-thick polyethylene $\left(\mathrm{CH}_{2}\right)$ analyser placed before the first set of straw chambers. It is common with such devices to speak of the Figure-Of-Merit (FOM), which is simply a combined measure of efficiency and analysing power. The efficiency arises from the effectiveness and geometric size (relative to the analysers) of the tracking devices, whereas the analysing power is a property of the analyser material and is related to the strength of the spin-orbit coupling. The addition of the $\mathrm{CH}_{2}$ analyser was motivated by a desire to increase the overall FOM by effectively producing two independent polarimeters.

\subsubsection{Choice of Analyser}

For safety, cost and efficiency considerations carbon was originally chosen as the standard analyser material in the FPP. It has a maximum thickness of $513 \mathrm{~mm}$ and is composed of five individual blocks of high purity graphite (each with a different thickness). As experiments have moved to taking data at higher proton momenta ( $>2.4 \mathrm{GeV} / \mathrm{c}$ ), where the carbon analysing power begins to fall sharply, other alternatives have been considered. In a recent double polarisation measurement of the proton form factors [70], where the proton momentum range was $2.6-3.8 \mathrm{GeV} / \mathrm{c}$, a $\mathrm{CH}_{2}$ analyser replaced the carbon for the first time. With the carbon completely removed, two blocks, amounting to a total thickness of $100 \mathrm{~cm}$ of $\mathrm{CH}_{2}$, were accommodated within the focal plane between the front and rear straw chambers. The resultant improvement in the FOM was significant compared to the use of carbon [71]. However, subsequent results from tests at Dubna [72] in this proton energy range demonstrated that a reduction in the total thickness of the $\mathrm{CH}_{2}$ to $40 \mathrm{~cm}$ had almost no impact on the size of the analysing power.

These two considerations, therefore, led to the conclusion that the optimum device for measurements at higher proton momenta would consist of two analysers in the focal plane, and utilise the pre-existing proton trajectory data obtained from the VDC's. With minimal hardware changes involving re-positioning of components, a dual analyser configuration of the FPP (see fig. 4.7) was used to extract the polarisation observables in the present measurement. In this arrangement, the protons can be scattered in either the $\mathrm{CH}_{2}$ or carbon analysers, with 
the VDC's and front and rear straw chambers used to track the proton before, between and after the analysers.

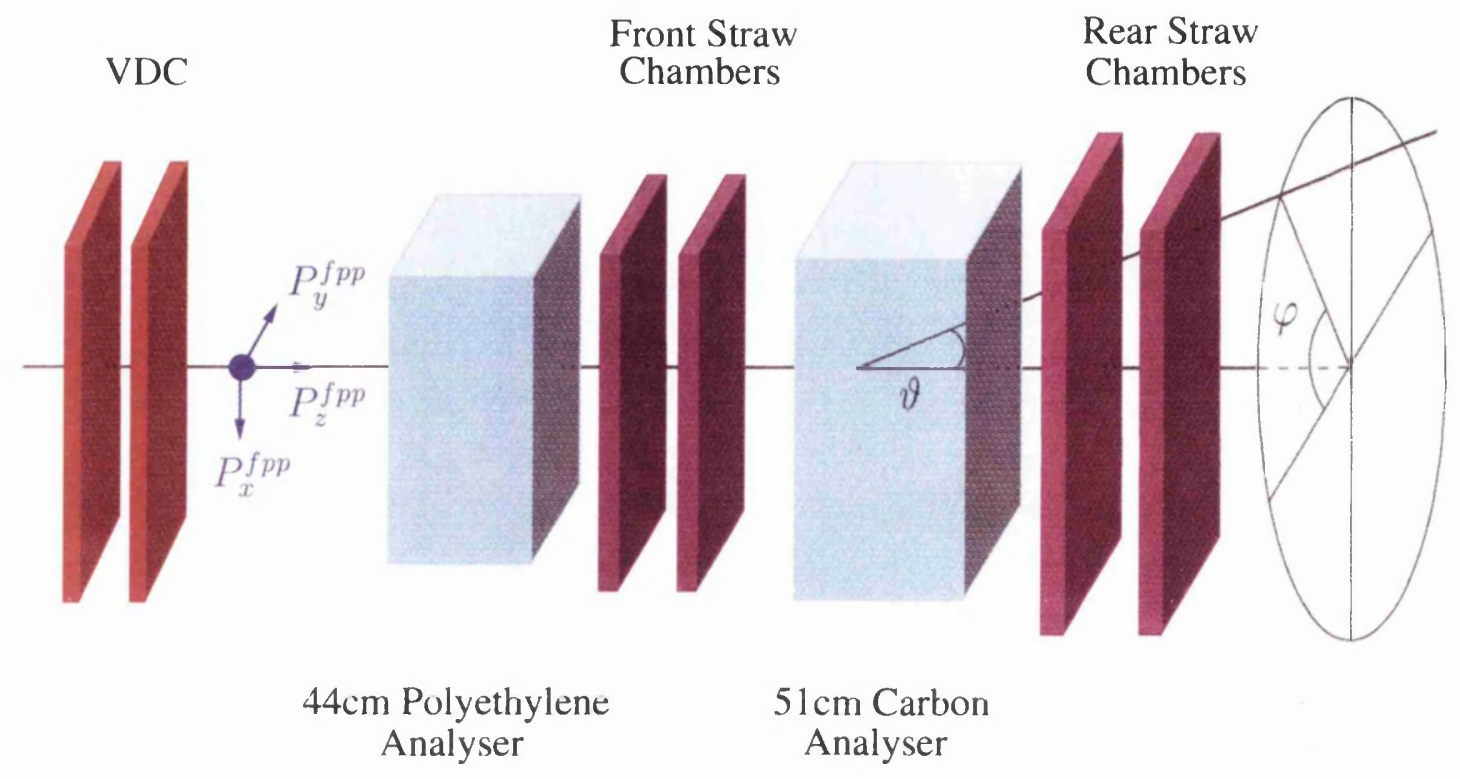

Figure 4.7: Dual analyser configuration of the Hall A FPP. Also shown in the diagram are the definitions of the focal plane proton polarisation ( $\left.\mathbf{P}^{\mathrm{fpp}}\right)$, and the polar $(\vartheta)$ and azimuthal $(\varphi)$ scattering angles.

\subsubsection{Straw Chambers}

Reconstructing the scattering angles in the analyser material requires precise knowledge of the proton trajectories before and after the analyser. Two sets of FPP straw chambers were originally built for this purpose and in the present experiment, they, together with the standard HRS VDC's (sec. 4.2.4), are used for polarimeter tracking.

Chambers 1 and 2 form the first (front) pair and are identical in design. To provide redundancy they consist of three $u$ and three $v$ planes, orientated at $+45^{\circ}$ and $-45^{\circ}$ to the dispersive (vertical or $x$ ) direction. The rear chambers, by comparison, are significantly larger than their front counterparts to ensure almost complete coverage for events scattered in the carbon with a polar angle $<20^{\circ}$. The six planes which make up chamber 4 have the same orientation as the front chambers, but, to resolve events where there may be multiple tracks, chamber 3 was designed with two $u$, two $v$ and two $x$ planes. The active area covered by each chamber is given in table 4.3 . 


\begin{tabular}{|l|c|}
\hline FPP Chamber 1 & $209 \times 60 \mathrm{~cm}$ \\
\hline FPP Chamber 2 & $209 \times 60 \mathrm{~cm}$ \\
\hline FPP Chamber 3 & $268 \times 124 \mathrm{~cm}$ \\
\hline FPP Chamber 4 & $298 \times 144 \mathrm{~cm}$ \\
\hline
\end{tabular}

Table 4.3: Active area covered by the four FPP straw chambers.

Each plane consists of a set of cylindrical tubes (straws) of radius $5 \mathrm{~mm}$ with a thin wire strung along the axis of each tube. A high voltage of $\sim 1.9 \mathrm{kV}$ is applied to each wire, and a gas with a similar mixture to the VDC's is flowed through each individual straw. These chambers work on the same principle as the one described previously in sec. 4.2.4. A proton ionises gas atoms leaving a trail of electrons which produce a negative pulse on the wire at the centre of each tube. Because the tubes form a physical ground, only one wire per plane will have a signal for a particular proton track. Therefore, signals from eight adjacent wires can be multiplexed, allowing a significant reduction in the required frontend electronics and overall cost. Each of the eight multiplexed channels has a different logic output pulse width associated with it, and both leading-edge and trailing-edge times of this logic pulse are recorded by a LeCroy 1877 TDC. Thereby, the drift distance can be calculated from the leading-edge drift time and drift velocity, whereas the individual wire hit can be recovered from the difference between leading-edge and trailing-edge time.

\subsection{RCS Photon Spectrometer}

The Hall A photon spectrometer was custom-built by the RCS collaboration for the specific purpose of detecting the scattered photon in this experiment. It was designed to have excellent position resolution to discriminate between Compton photons and those produced in background processes, and also provide a means of identifying, and rejecting, the kinematically indistinguishable events from elastic $e p$ scattering. The requirement for good position resolution was also consistent with the need for high granularity to keep counting rates in individual elements at a manageable level. The spectrometer that was ultimately used in the experiment had two components: a highly segmented lead-glass electromagnetic calorimeter and a deflection magnet. 


\subsubsection{Electromagnetic Showers}

As discussed in sec. 4.2.2, the dominant process by which high energy electrons lose energy is bremsstrahlung radiation. In comparison, the prevalent interaction for high energy photons is electron-positron pair production. By these two mechanisms, an electron or photon interacting with matter will produce a shower of lower energy photons and electrons until the energy falls below some critical value dependent on the properties of the material. The resultant cascade of photons and electrons is known as an electromagnetic shower, and devices which accumulate the incident photon/electron energy by these processes are known as electromagnetic calorimeters.

The longitudinal evolution of the shower is described in terms of the radiation length of the material in which the shower evolves. After one radiation length a bremsstrahlung or pair production interaction leads to a new generation of particles being produced. The critical energy of the shower constituents, $\epsilon_{0}$, corresponds to the pair production threshold, below which energy loss by ionisation begins to dominate. In a simple model of shower development the penetration depth is given, in terms of number of radiation lengths, by

$$
n_{X}=\frac{\ln \left(\frac{E_{0}}{\epsilon_{0}}\right)}{\ln 2}
$$

where $E_{0}$ is the initial electron or photon energy. The other key material dependent property, which characterises the transverse spread of the shower, is the Moliére radius. The lateral spread of an electromagnetic shower primarily emanates from Coulomb scattering of the shower electrons.

The best materials for electromagnetic calorimeters are those with high atomic number, and in this particular instance lead-glass (type TF-1) was used. Its relevant properties [73] are shown in table 4.4. Any charged particle traveling in an optically transparent material with a velocity greater than that of light in the material produces Cerenkov radiation. This radiation takes the form of optical photons in the lead-glass, the total number of which depends on the number of particles in the shower with velocity $>c_{\text {lead-glass }}$. By detecting and integrating these photons in a Photo-Multiplier Tube (PMT), the total energy in the shower can be reconstructed with reasonable precision. Where the calorimeter granularity is sufficiently high so that the lateral spread of the shower penetrates several adjacent blocks, the centre of gravity of the shower can be used to determine the 
hit position to much better precision than the physical size of the blocks.

\begin{tabular}{|l|c|}
\hline Density $\rho$ & $3.86 \mathrm{gcm}^{-3}$ \\
\hline Refractive Index $n$ & 1.65 \\
\hline Radiation Length $X_{0}$ & $2.5 \mathrm{~cm}$ \\
\hline Moliére Radius $R_{M}$ & $3.3 \mathrm{~cm}$ \\
\hline Critical Energy $\epsilon_{0}$ & $15 \mathrm{MeV}$ \\
\hline
\end{tabular}

Table 4.4: Physical properties of TF-1 lead-glass.

\subsubsection{Electromagnetic Calorimeter}

A group led by the Yerevan Physics Institute designed and built the RCS total absorption electromagnetic calorimeter. It is made up of a total of 704 lead-glass blocks, with a FEU-84/3 PMT optically coupled to the rear of each biock. The lead-glass is arranged in 22 columns and 32 rows, as shown in fig. 4.8 , leading to a cross-sectional area of $128 \times 88 \mathrm{~cm}^{2}$. It is housed within a light-proof containment structure with interlocked doors at the rear for easy access to the PMT's.
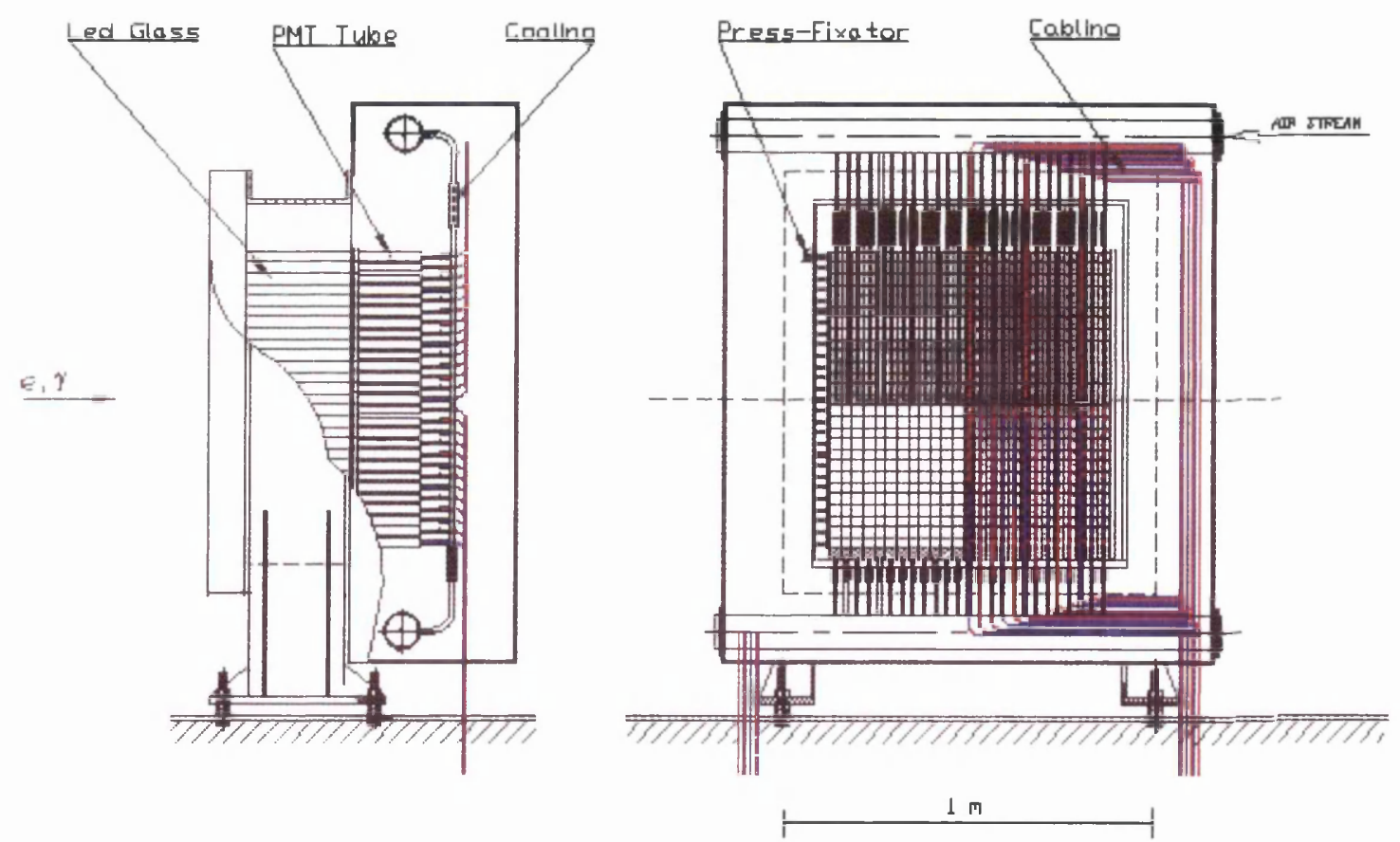

Figure 4.8: Diagram of the RCS calorimeter which shows the arrangement of the lead-glass blocks, the forced-air cooling system and the cabling, within the support structure. 
From the angular resolution requirements and the lead-glass properties shown in tab. 4.4, the individual blocks were chosen to have the dimensions $4 \times 4 \times 40 \mathrm{~cm}^{3}$. They were wrapped in aluminised Mylar film and black Tedlar to ensure there was no exposure to external light, which could seriously damage the PMT's. Individual tubes were coupled to the lead-glass by optical grease and pressed into contact by springs attached between a grid of steel supports and the base of the tube.

The PMT is one of the essential tools in experimental physics. It is used to produce and amplify an electrical signal from optical photons, in this particular case from Cerenkov radiation in the lead-glass. The tube, kept under vacuum, consists of an optical window followed by a photocathode, which emits electrons when photons strike it with a typical quantum efficiency of $20-25 \%$. The electrons are then focused and accelerated through a series of dynodes producing secondary electron emission and hence amplification. The derived electron signal is ultimately collected on an anode located at the opposite end of the PMT relative to the photocathode. Proper operation of the tube requires reasonably high voltage gradients between dynodes, and in the present case a total voltage difference of $1600 \mathrm{~V}$ was applied. The electrical output from each tube is sent to trigger logic and a Fastbus 1881 Analogue-to-Digital Converter (ADC), which digitises the charge accumulated over a specific time interval (the gate).

Because the gain response of each PMT is not identical, different tubes require different high voltages to produce the same signal amplitude for a given amount of incident light. A gain monitoring system was designed for calibration of the multiple high voltage channels supplying the calorimeter. To expose each PMT to approximately equal light levels, pulsed ultra-violet light from a nitrogen laser was incident on a plastic scintillator, acting as a wavelength shifter and removing the directionality of the laser light, the output from which was then transported in a series of optical fibres to a plate of the transparent plastic Lucite. The calorimeter was illuminated by the light from this plate, which was located immediately adjacent to the calorimeter face, spanning its full cross sectional area. The process was fully automated, and operated remotely using Java control software and a LeCroy-1458 based high voltage system.

A support structure was designed to accommodate the calorimeter, the accompanying front-end electronics, the gain monitoring system, the forced-air PMTbase cooling system and the large amount of cables. It also facilitated movement of the entire assembly between the various kinematic points. Also for ease of 
movement the signal and high voltage cables (over two thousand of them) were placed on a series of bogies at the rear of the calorimeter platform. Finally, small light sources at the front and rear of the support platform shining vertically onto the hall floor were used to align the photon-arm to marks painted on the floor. Only moderate accuracy of the positioning of the calorimeter was required, since the difference between expected and measured photon hit position, rather than the absolute position itself, is used in the event identification process. The marks for each kinematic point, produced by a survey of the experimental hall, provided the desired accuracy while allowing a simple and flexible method for positioning of the spectrometer.

\subsubsection{Electron Rejection}

The mixed electron/photon beam was unavoidable due to luminosity considerations. Consequently, however, it made necessary the identification and rejection of elastic ep scattering events. This is due to the fact that the calorimeter alone provides no means to distinguish whether a shower has been induced by a primary electron or photon hit, and the $\gamma p$ and $e p$ reactions are kinematically indistinguishable. Therefore, two distinct steps were taken:

1. Avoiding the large electron flux at the bremsstrahlung endpoint (as discussed in sec. 4.2.2).

2. The design and building of a deflection magnet.

The two-body kinematic relationship that describes both Compton and elastic $e p$ scattering allows one to use the detected proton data to predict the angle at which the expected partner photon or electron will be detected. Inserting a magnetic field between the target and calorimeter, which will obviously deflect the negatively charged electrons while leaving the Compton photons undeflected, means this kinematic correlation will no longer hold for the ep events. This was the principal means by which the $\gamma p / e p$ separation was achieved at all points in the experimental program. To keep costs low it was decided that a magnetic deflection of around $10 \mathrm{~cm}$ on the calorimeter face when it was at its closest position to the target was sufficient. In addition, since the magnetic field would be used purely to destroy the $e p$ kinematic correlation, and not for any reconstruction purposes, a simple dipole magnet with a field perpendicular to the hall floor (in the positive $y$ direction) was entirely adequate. The measured field map of the 


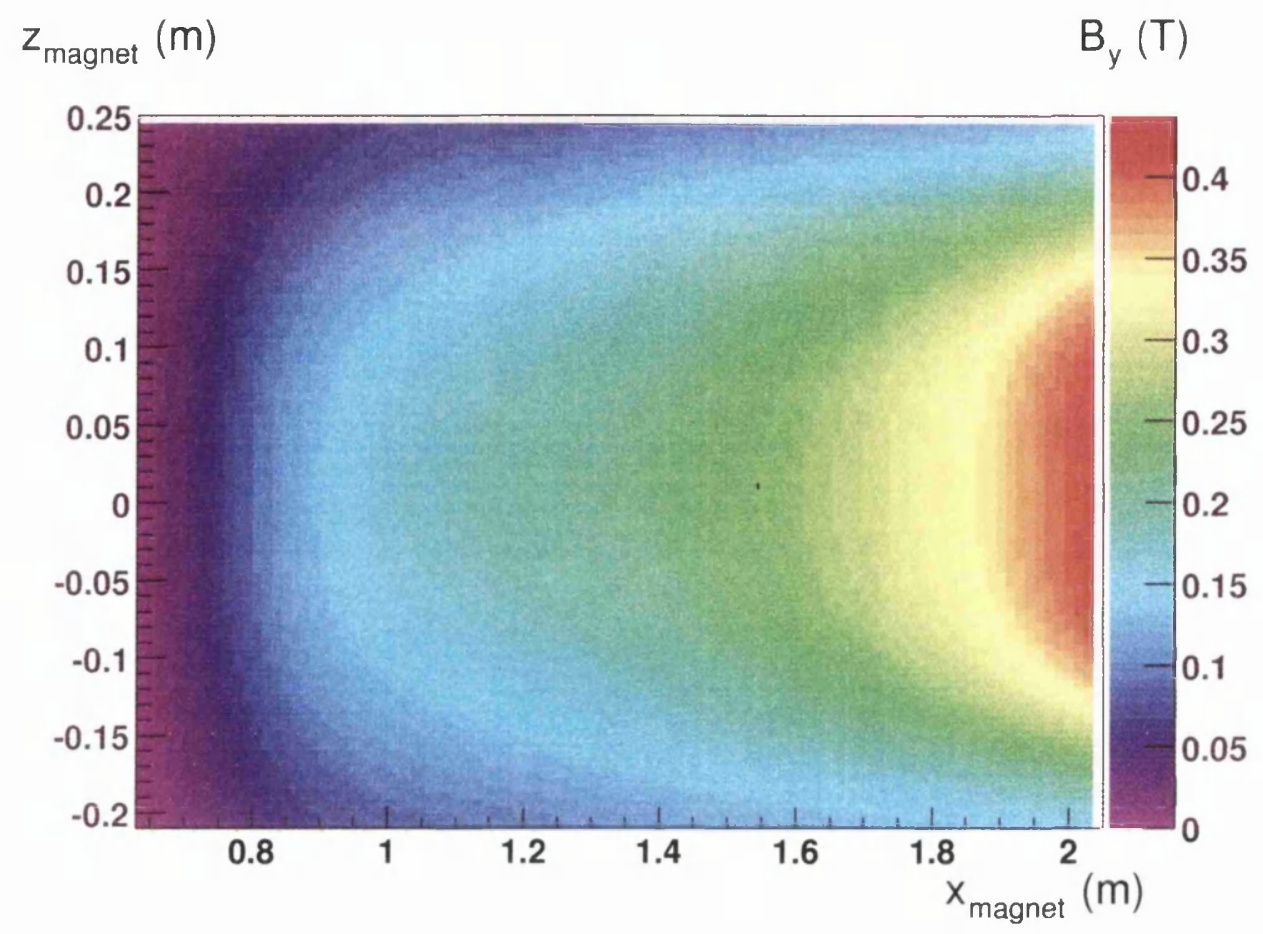

Figure 4.9: A map of the measured y-component of the deflection field as a function of $x_{\text {magnet }}$ and $z_{\text {magnet }}$.

RCS magnet |74| as a function of the horizontal $x-z$ coordinates for $y$ centered between the pole faces is shown in fig. $4.9^{2}$. This shows, crucially, that the field is not uniform: its magnitude becomes larger as $x_{\text {magnet }}$ increases to compensate for the fact that electrons which elastically scatter at larger angles (and therefore at larger $x_{\text {magnet }}$ ) have higher momentum than those which scatter at smaller angles.

\subsection{Data Acquisition}

The standard Hall A DAQ system consists of a variety of CAMAC, NIM, Fastbus and VME electronics, as well as modules purpose built by the JLab electronics group. Software control and management of the hardware components is implemented by the DAQ toolkit CODA (CEBAF On-line Data Acquisition [75]).

In the current experiment, the hadron arm trigger and readout electronics were part of the standard Data Acquisition (DAQ) system associated with each

\footnotetext{
${ }^{2} z_{\text {magnet }}$ points toward the centre of the calorimeter and $x_{\text {magnet }}=y_{\text {magnet }} \times z_{\text {magnet }}$.
} 
HRS (there are also separate systems for the various beamline monitoring devices described in sec. 4.2.1). The inclusion of the photon spectrometer required a completely new readout package, for which a new trigger scheme had to be designed. The hadron readout electronics were located within the shielding hut above the focal plane detectors in the HRS-left, while the photon arm electronics were positioned near the HRS on the left-hand side of the hall floor (the opposite side to the photon detector). The radiation levels within Hall A are sufficient to disrupt the operation of micro-electronic circuits and could cause long-term damage. To counter this, a large concrete shield was constructed in front of the photon-arm electronics.

\subsubsection{Trigger Scheme}

The photon trigger is provided by the PMT signals from individual electromagnetic calorimeter blocks, which are summed in the manner highlighted in fig. 4.10. The electrical signals from each of the $2 \times 4$ sub-arrays of adjacent lead-glass blocks, excluding the outermost layer, are summed in a linear-summing module to give a $s_{4} m_{8}$ signal. These, in turn, are further summed in overlapping groups of four to give a $s_{32}$ signal, of which there are 56 in total. The $s_{32}$ signals are sent to discriminators which produce a logic pulse if the input signal is above a given magnitude. The photon singles trigger ( $\mathrm{T} 1$ ), formed by taking the logical OR of the $s_{32}$ signals, is produced only when the total energy accumulated in the calorimeter is above a certain value (chosen to be around $300 \mathrm{MeV}$ ). The associated electronics are illustrated on the top left-hand side of fig 4.11.

Passage of a charged particle through an organic scintillator causes light production which can be detected and accumulated in a PMT. The response of such detectors is extremely fast, so very good timing resolution is possible. For these reasons, scintillators are commonly used to produce a trigger signal for composite detector systems. This is indeed the case in the HRS-left, where three scintillator planes are located in the focal plane detector stack (fig. 4.5). The coincidence signal between the hadron and photon signals was formed at the photon arm electronics, away from the HRS focal plane, which meant that SO was favored over the traditional S1/S2 combination. The S0 scintillator plane is comprised of a single $10 \mathrm{~mm}$-thick plastic scintillator paddle viewed by two PMT's at opposite ends of the paddle. The logical AND of the signals from both tubes gives the proton singles trigger (T7). 


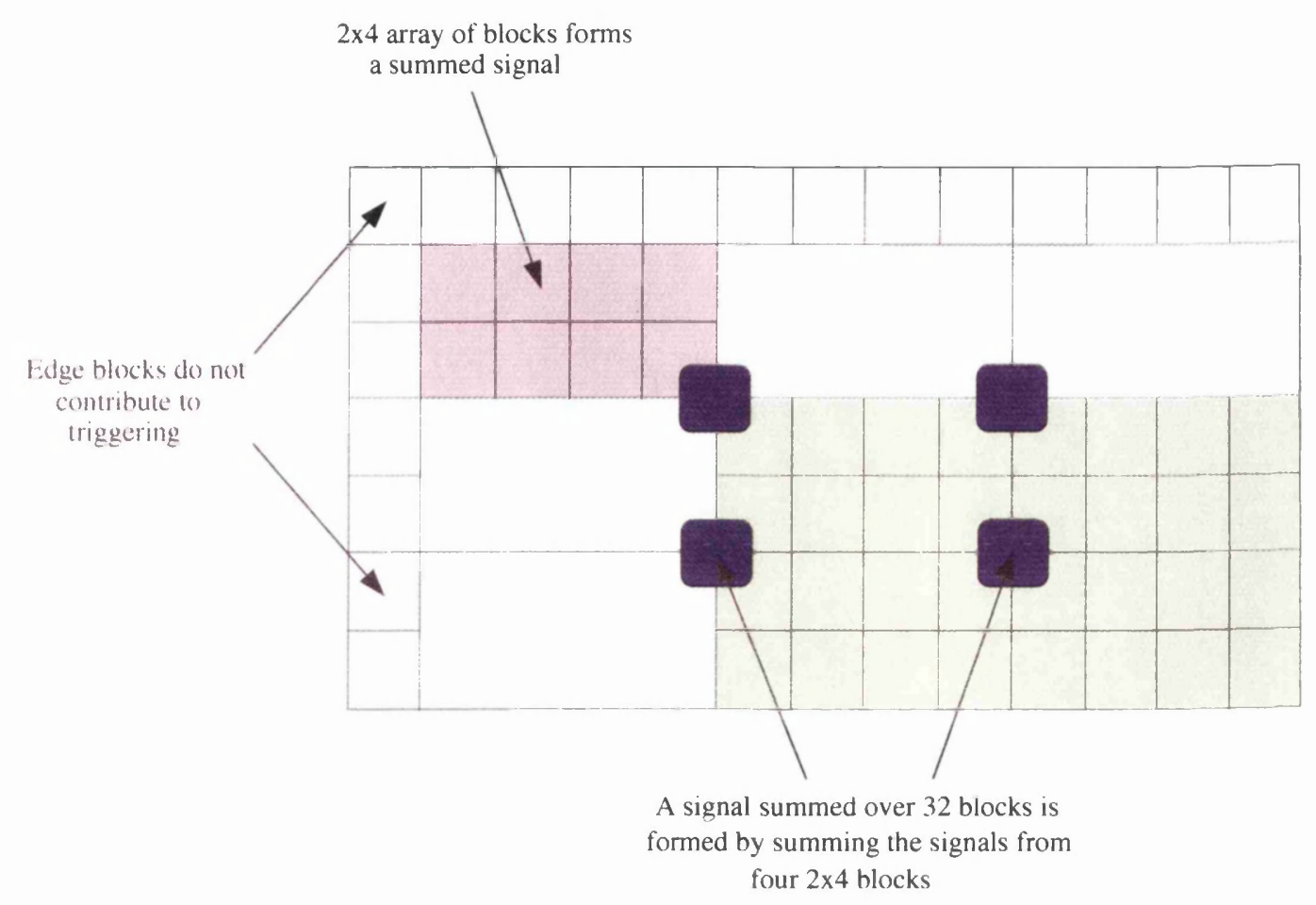

Figure 4.10: The photon trigger is made up of the $O R$ of 56 overlapped sum s $_{32}$ signals, which are formed by summing signals from adjacent calorimeter blocks.

A schematic of the complete trigger logic is shown in fig. 4.11. To ensure an actual coincidence event will produce a coincidence trigger, the timing relationship between the $\mathrm{S} 0$ and calorimeter triggers was measured and the signals aligned in time using programmable delay units (for simplicity, these delays are not shown in the figure). For different kinematics, the relative delays were calculated on the basis of the proton and photon time-of-flight so that tight coincidence windows could be applied. The width of the coincidence window is defined by the S0 trigger, which was set to be $100 \mathrm{~ns}$. The T5 coincidence trigger is then formed when a photon trigger (set to be only $10 \mathrm{~ns}$-wide) is produced within this window.

\subsubsection{The Data Readout Software}

The physical information of interest is obtained from raw data (digital numbers) read out from thousands of ADC and TDC channels. Readout from these individual channels into the data-stream, ultimately to be written to tapes in the JLab mass storage system, is controlled by a CODA-based system configured for the current experiment. ADC and TDC modules for the whole setup were located in four Fastbus crates, each controlled by a single-board computer with a Fastbus 


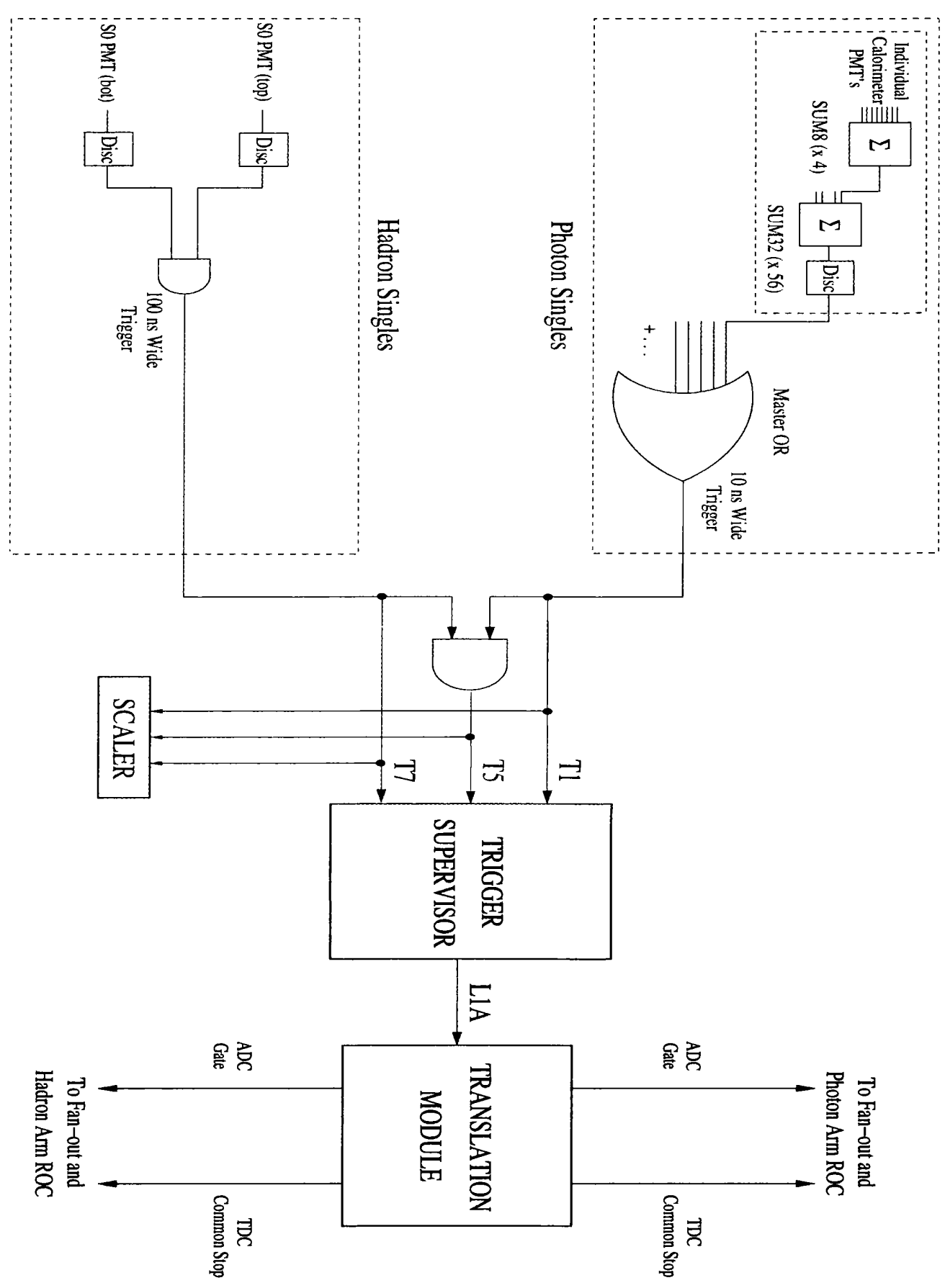

Figure 4.11: A schematic of the full trigger electronics for the RCS experiment. The formation of the photon and hadron triggers, in addition to a description of the various components, is described fully in the text.

interface called a ReadOut Controller (ROC). A VME crate located in the photon arm electronics contains the Trigger Supervisor (TS) and Event Builder (EB) modules, which together are responsible for overall readout and organisation of 
the final data-set.

The trigger supervisor is a $6 U$ VME board custom built by the JLab electronics group [76] and controlled by a Motorola MVME 2400. It can accept up to 12 independent triggers, to each of which programmable pre-scale values may be applied. Furthermore, for each of the triggers the TS produces an event type tag, included in the data stream, and a latch pattern which is recorded in a separate TDC module. For production running, coincidence events (T5) were obviously the most important, and all other trigger types were given very large pre-scale values. However, for cosmic data-taking and various calibration processes during beam-off periods, these pre-scale factors were adjusted to include other trigger types.

In the current configuration, each accepted trigger (after pre-scaling) outputs a Level 1 Accept (L1A) signal from the trigger supervisor. This is sent to all ROC's, where it is used to provide gates for the ADC modules and to give common stop signals to the TDC's. After a module receives a L1A signal and finishes handling the front-end data a busy flag is cleared in the Fastbus crate. The ROC then communicates to the TS that it is ready to accept another trigger. The Fastbus 1877 TDC modules used in this experiment have 96 channels and are capable of detecting multi-hit events. They have a time resolution of $1 \mathrm{~ns}$ and are operated in common-stop mode. In this mode an event, such as a calorimeter hit, starts the appropriate TDC's counting, and they are only stopped and read-out if the particular event produces an associated trigger (and L1A signal). The Fastbus 1881 ADC modules have 64 channels, each of which has a separate threshold which is set remotely. The total charge accumulated from the signal pulse during the gate period is measured and read out. The gate period for all ADC's was set to 100 ns. 


\section{Chapter 5}

\section{Data Analysis: Event Selection}

The unique nature of the RCS experiment motivated a highly modular approach to the data analysis. This work can be neatly separated into two parts: the selection of Compton events over other competing reactions and the extraction of the recoil proton polarisation transfer components. This chapter will deal with the former, which is common to both polarisation and cross section measurements. The much more specialised polarisation analysis will be covered in the following chapter.

\subsection{Overview}

Three software packages were used for this analysis, although very little actual software details are given here. Instead, emphasis is given to the appropriate calculations and the principles behind them. The common features of data analysis applications of this type are:

- Decoding the raw data from individual ADC and TDC channels.

- Calibration into more meaningful numbers by using pre-determined constants.

- Reconstruction of the physical parameters of interest (e.g. a VDC track or electromagnetic shower).

- Storage of the reduced data-set in an easily accessible format for histogramming and further calculations. 
The reconstruction of physical parameters is by far the most important attribute and contains all the appropriate physics calculations.

For hadron analysis, the key reconstruction stages are the VDC tracking at the HRS focal plane and relating this information to the initial proton track at the target. Similarly, reconstruction of electromagnetic showers in the calorimeter, specifically the position of the origin of these showers, is the most important aspect of the photon arm analysis. The complementary properties associated with both particles are then combined with the trigger information and a series of kinematics calculations to help identify the source of a particular event. These kinematic calculations are fairly detailed so they have been included, together with a definition of the various coordinate systems used in the analysis, in Appendix A.

A range of kinematic settings and experimental conditions were used in the course of the full RCS experiment, only two of which are important in the calculation of the RCS polarisation observables:

1. $3 E$ Off-endpoint: The data taking period for producing RCS events and measuring the associated polarisation transfer components. The target with the bremsstrahlung radiator was used, the spectrometer positions were selected to cover the required incident energy range and the photon-arm magnet was used to help separate elastic ep events.

2. $3 D$ Endpoint: In these runs elastic electron-proton $(e p)$ scattering data was accumulated for FPP calibration. A mixed electron/photon beam was not desired at this stage, so the bremsstrahlung radiator was removed between the beam and target. In addition, since no electron rejection was required, the photon-arm magnet was switched off.

In all following discussions, the data corresponding to these two sets of experimental conditions will simply be referred to as production and calibration data, respectively.

\subsection{Hadron Analysis}

For all previous Hall A experiments, the software package espace (Event Scanning Program for hall A Collaboration Experiments) [77] has been used for data analysis. It is a Fortran 77 based code with DEC VMS extensions [78], which utilises 
many of the data manipulation and display tools that are part of the widely-used CERNLIB package [79]. It involves techniques for the HRS data analysis which, as a result of copious use in recent years, are stable and well-tested. The principal element is VDC tracking and the closely related magnetic reconstruction of the recoil proton momentum, direction and reaction vertex at the target. Besides this, it incorporates beamline diagnostics (i.e. those described in sec. 4.2.1), of which the BPM data is most critical in trajectory reconstruction. As in the experimental situation espace is conventionally used with a dual HRS configuration.

\subsubsection{VDC Track Reconstruction}

In the Hall A VDC's, the time taken for electrons produced by ionisation along a proton track to reach the anode wire is measured by TDC's and converted into perpendicular distance from the wire. Since the hit time in a particular TDC depends on different cable lengths and signal processing times, which may differ from channel to channel, a reference time $\left(t_{0}\right)$ must be found for each wire. This is used to extract the drift time from the actual TDC hit, which can then be converted to drift distance through calibration based upon measurements of the drift velocity [65].

The next stage involves identifying and calculating the best available tracks from a linear fit of the drift distances for adjacent wires, for which one can obtain the local cross-over points and local trajectory angles. Determination of the crossover point is unambiguous, whereas the value obtained for the local trajectory angle depends strongly on the reconstruction algorithm. This quandary can be avoided by calculating general trajectory angles from the cross-over points of a particular track on planes in two different VDC's. Hence, a trajectory is defined (relative to the some reference wire and plane) by two positions and two angles:

$$
\left(U, V, \eta_{U}, \eta_{V}\right)
$$

Finally in this process, this reconstructed VDC trajectory must be expressed in a manner which allows one to determine the corresponding trajectory at the target through the optical transfer properties of the HRS. Therefore, the track variables must be transformed from the local VDC coordinate system to the Transport Coordinate System (TRCS). 


\subsubsection{Target Coordinate Reconstruction}

In the transport coordinate system the trajectory of the proton at the focal plane, at the target and through the HRS magnetic elements is described by a vector $(\mathbf{t})$, which expresses the track relative to the central reference trajectory. This vector is characterised by five components:

$$
\mathbf{t}=\left[\begin{array}{l}
x \\
\theta \\
y \\
\phi \\
\delta
\end{array}\right]
$$

where:

- $x$ is the dispersive (vertical) displacement from the central trajectory, expressed in metres.

- $\theta$ is the angle the trajectory makes in this plane relative to the central trajectory $(d x / d z)$, expressed in radians.

- $y$ and $\phi$ are the equivalent displacement and angle in the non-dispersive (horizontal) plane.

- $\delta$ is the fractional deviation of the momentum from the central value ( $\Delta p-$ $\left.p_{0} / p_{0}\right)$, expressed in percent.

In a first-order approximation, a transport matrix can be defined to relate the measured focal plane coordinates to their counterparts at the target. This matrix involves only four unknown parameters since $x_{t g t}$ is known from the BPM data (sec. 4.2.1):

$$
\left[\begin{array}{c}
\delta \\
\theta \\
y \\
\phi
\end{array}\right]_{t g t}=\left[\begin{array}{cccc}
\langle\delta \mid x\rangle & \langle\delta \mid \theta\rangle & 0 & 0 \\
\langle\theta \mid x\rangle & \langle\theta \mid \theta\rangle & 0 & 0 \\
0 & 0 & \langle y \mid y\rangle & \langle y \mid \phi\rangle \\
0 & 0 & \langle\phi \mid y\rangle & \langle\phi \mid \phi\rangle
\end{array}\right]\left[\begin{array}{c}
x \\
\theta \\
y \\
\phi
\end{array}\right]_{f p}
$$


The situation in practice, however, is far more complicated, with four transfer tensors - one for each of the trajectory parameters - replacing the matrix in eq. 5.2. These tensors are obtained, together with any position offsets of the spectrometer or VDC's relative to their nominal (central) positions, using an optics optimisation procedure. If the four tensors are labeled $Y_{j k l}, T_{j k l}, P_{j k l}$ and $D_{j k l}$, their elements are polynomials in $x_{f p}$ which are determined up to fifth order, and relate focal plane and target quantities according to:

$$
\begin{aligned}
y_{t g t} & =\sum_{j, k, l} Y_{j k l} \theta_{f p}^{j} y_{f p}^{k} \phi_{f p}^{l}, \\
\theta_{t g t} & =\sum_{j, k, l} T_{j k l} \theta_{f p}^{j} y_{f p}^{k} \phi_{f p}^{l} \\
\phi_{t g t} & =\sum_{j, k, l} P_{j k l} \theta_{f p}^{j} y_{f p}^{k} \phi_{f p}^{l} \\
\delta_{i} & =\sum_{j, k, l} D_{j k l} \theta_{f p}^{j} y_{f p}^{k} \phi_{f p}^{l} .
\end{aligned}
$$

The procedure for optimising these tensors [80] involves detecting electrons which undergo quasi-elastic scattering from the optics target: one of the elements on the target ladder, which consists of a stack of thin ${ }^{12} C$ foils. This target is used because the intersection point of the incoming electron beam and any one of these foils effectively provides a point target.

During the present optics data-taking period, in addition to installing the optics target, a 49 hole sieve-slit collimator was placed at the entrance to the HRS-left aperture and the polarity of the magnets was switched. Each target foil position along the beam axis $\left(z_{\text {react }}\right)$ and the positions of the centre of the sieveslit holes $\left(x_{\text {sieve }}\right.$ and $\left.y_{\text {sieve }}\right)$ must be known very accurately, so they were surveyed at the beginning this period. The coefficients of the polynomials in eq. 5.3 can then be determined from this optics data by minimising the difference (via $\chi^{2}$ ) between the reconstructed and surveyed target foil and sieve-slit hole position. Fig. 5.1 shows the reconstructed sieve-slit distribution after optimisation, along with the physical layout of the collimator for comparison. Also shown in this figure are typical distributions of the four reconstructed quantities describing the proton trajectory at the target. 

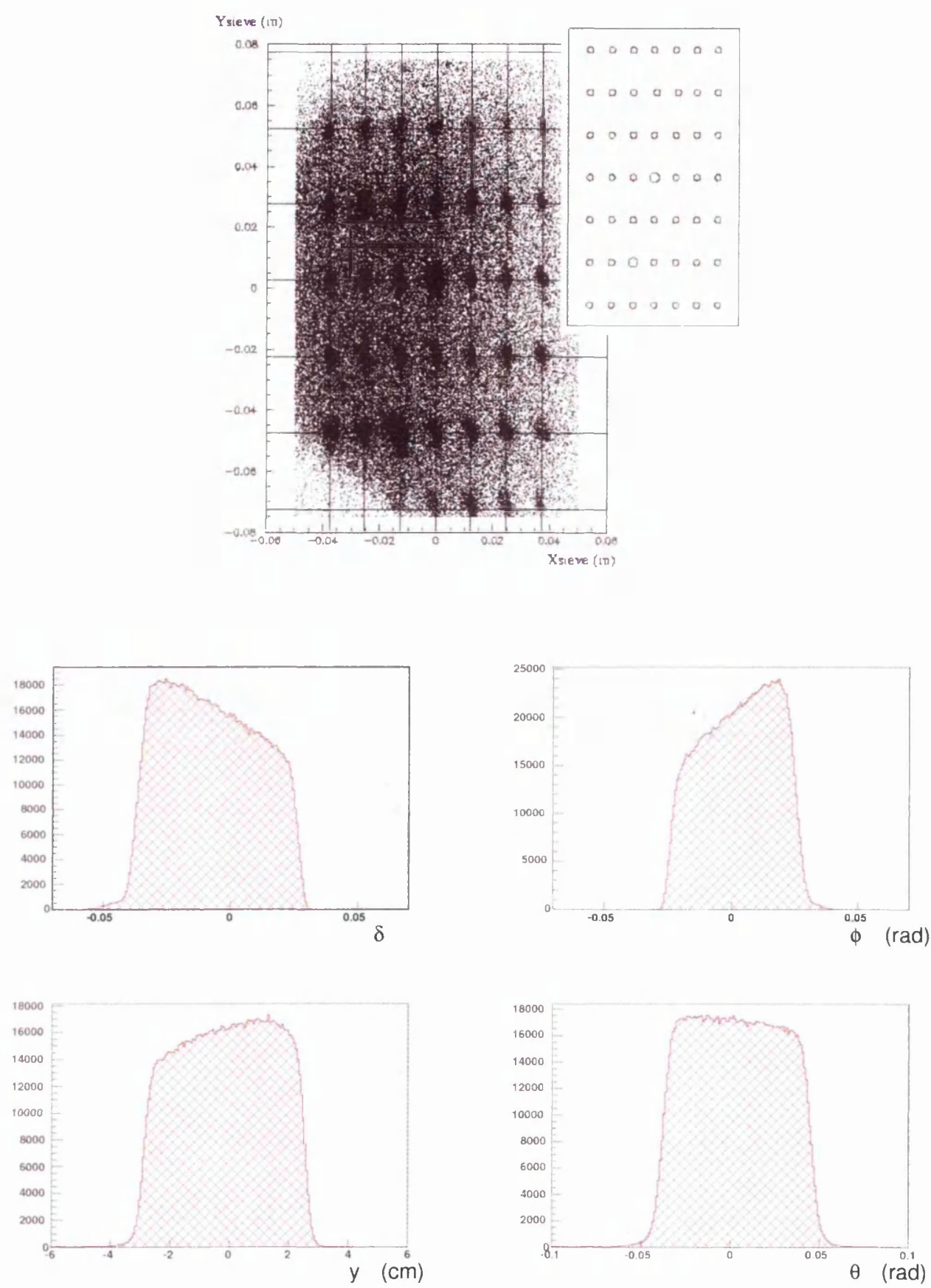

Figure 5.1: Data analysed in espace after optimisation of the HRS optics tensors. Top: optics data with reconstructed sieve-slit position. Bottom: transport system proton trajectory parameters, $\delta, \phi_{t g t}, y_{t g t}$ and $\theta_{t g t}$, taken from calibration data. 


\subsection{Photon Analysis}

Since the photon calorimeter was being used for the first time, new software had to be written to reconstruct electromagnetic showers in the calorimeter from the raw data. The decision was made not to include this in espace, but to design a new application in the framework of the Hall A ROOT/C ++ architecture [81, 82], which is currently in the process of superseding espace. The most important aspect of the shower reconstruction, made necessary by the need for good angular resolution, is the calculation of the hit position of the scattered photon/electron on the front face of the calorimeter.

\subsubsection{Calorimeter Shower Reconstruction}

If a high energy photon or electron hits the centre of a calorimeter block its energy is deposited over an array containing the struck element and its immediate neighbours. For the RCS electromagnetic calorimeter which has a lateral block size of about one Moliére radius, a shower reconstruction involving energy deposition in two rings of blocks around the central element is required to separate events where there may be two or more showers. Typically around $80 \%$ of the incident energy is deposited in the central block, with less than $0.1 \%$ deposited in each corner block in a $5 \times 5$ matrix. This is emphasised in fig. 5.2 , which shows the simulated longitudinal development of an electromagnetic shower induced by a $1 \mathrm{GeV}$ electron incident on the central element.

The software conditions for identifying and reconstructing such an electromagnetic shower cluster were chosen to be:

1. A cluster is a group of adjacent calorimeter blocks with non-zero energy deposition.

2. The maximum number of blocks spanned by the cluster is 25 (i.e. a $5 \times 5$ matrix).

3. The shower profile (the distribution of energy deposited over the cluster blocks) must satisfy the following conditions:

(a) The central block is the element with the highest energy deposition.

(b) The energy deposited in the corner blocks must be less than in each of their neighbours. 


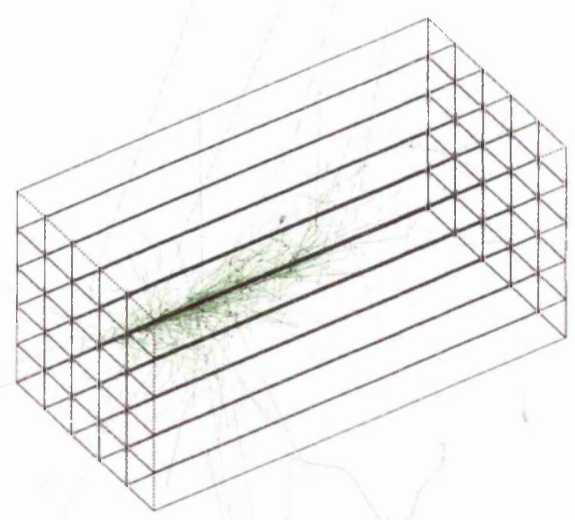

Figure 5.2: Event simulation for a sample electromagnetic shower using Geant4. The shower is induced by a $1 \mathrm{GeV}$ electron which is incident on the central block in a $5 \times 5$ array of lead-glass blocks.

(c) At least $50 \%$ of the total shower energy must be deposited in the central row (and, equivalently, in the central column).

From the raw ADC values recorded for the calorimeter blocks one can identify clusters and calculate the corresponding energy deposition and shower origin (centre of gravity). The fraction of energy deposited in each block which forms a cluster (of $M$ total blocks) can then be used in a geometric weighting algorithm which is linear in energy (another commonly-used method involves a logarithmic weighting) to find:

$$
\begin{aligned}
E_{\text {calo }} & =\sum_{i \in M} E_{i}, \\
x_{\text {calo }} & =\sum_{i \in M} \frac{E_{i} X_{i}}{E}, \\
y_{\text {calo }} & =\sum_{i \in M} \frac{E_{i} Y_{i}}{E},
\end{aligned}
$$

where:

- $E_{\text {calo }}$ is the total energy deposited in a cluster, and $E_{i}$ the energy deposited in the $i$ th block. 
- $x_{\text {calo }}\left(y_{\text {calo }}\right)$ is the $x(y)$ position of the shower centre of gravity, and $X_{i}$ and $Y_{i}$ are the physical coordinates of the $i$ th block.

\subsubsection{Calorimeter Energy Calibration}

To go from raw $\mathrm{ADC}$ values to the physical quantities in eq. 5.4 one must calibrate the energy conversion gains for each block in the calorimeter. Elastic $e p$ scattering data was taken several times at various stages of the experiment for this very purpose. Although the energy resolution of the calorimeter has been sacrificed somewhat in favour of position resolution, the two are clearly related (eq. 5.4). It will become obvious in the remainder of this chapter that detecting one of the particles in a two-body reaction allows one to reconstruct the other via kinematic calculations (Appendix A). So, reconstruction of the four-momentum of the proton as described in the previous section means that the corresponding scattered electron energy can be predicted. For energy calibration of the calorimeter a $\chi^{2}$ function, based upon the difference between the measured and predicted energy, can be defined and a minimisation procedure can be applied to obtain the optimal calibration.

The first step involves using the predicted electron energy $\left(E_{0}\right)$ to extract the energy conversion gain coefficients $\left(C_{i}\right)$ for each of the calorimeter blocks. These coefficients relate the measured ADC value to the physical energy deposition, and are calculated by minimisation of the function:

$$
\chi^{2}=\sum_{n=1}^{N}\left[\sum_{i \in M^{n}} C_{i}\left(A_{i}^{n}-P_{i}\right)-E_{0}^{n}\right]^{2},
$$

where:

- $M^{n}$ is the set of hit block indices in a particular cluster (for a particular event $n$ ).

- $A_{i}^{n}$ is the measured ADC value of the $i$ th block.

- $P_{i}$ is the equivalent $\mathrm{ADC}$ pedestal (the channel corresponding to the $E=0$ point).

Once the energy gain coefficients have been found they are stored in an input database for the shower reconstruction analysis. Radiation damage to the leadglass blocks in the calorimeter caused degradation of the energy resolution as 


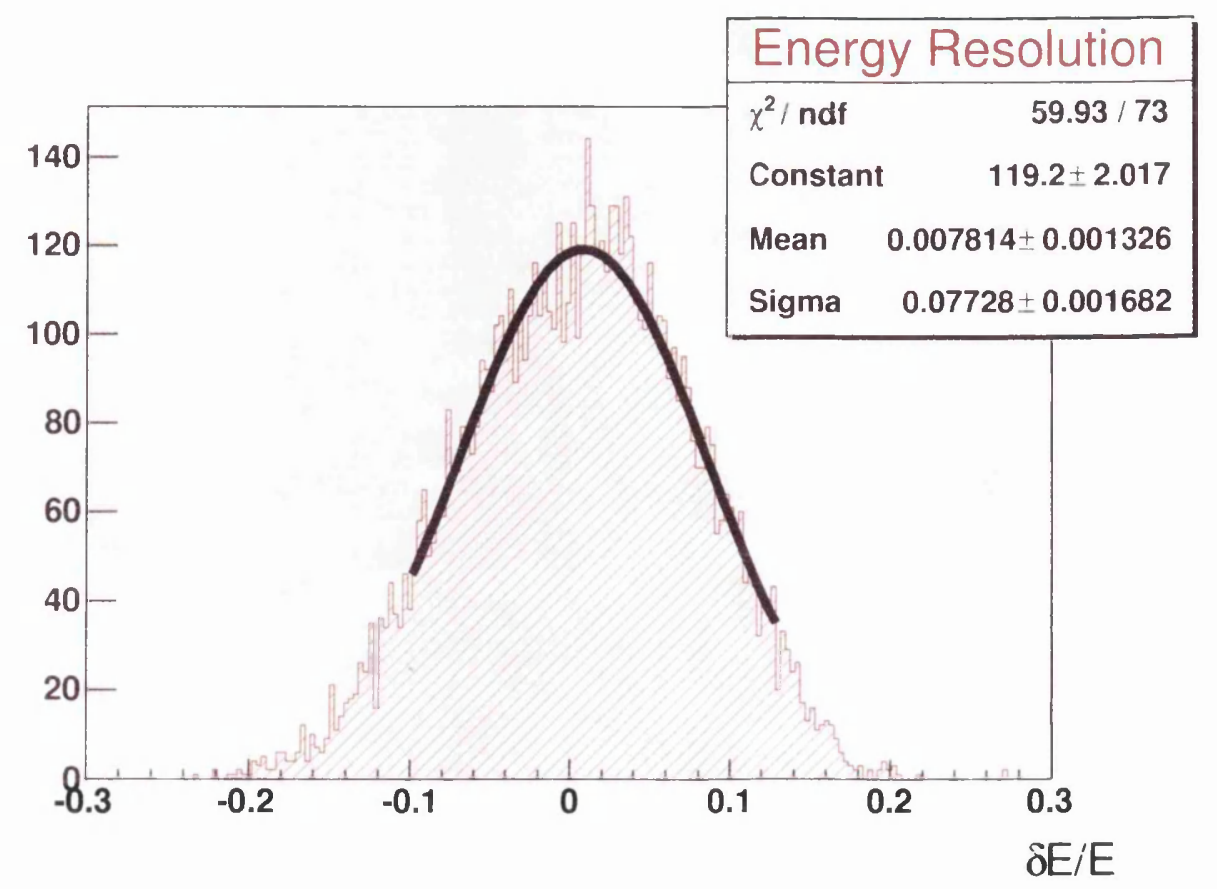

Figure 5.3: Spectrum of the difference between measured and predicted electron energy for calibration data after optimisation of the ADC conversion coefficients. The energy resolution is $7.7 \%$.

the experiment progressed. Interestingly, by the time the experiment came to an end, the total radiation dose was estimated to be $3-6 \mathrm{krad}$ and the energy resolution had fallen from $4.9 \%$ at the beginning to $\gtrsim 9 \%[83]$. The difference between measured and predicted electron energy for calibration data used in the current analysis can be seen in fig. 5.3. From this spectrum we see that the energy resolution after calibration was found to be $\delta E / E=7.7 \%$.

\subsection{Production Data}

Up to this point, we have dealt with the reconstruction of the physical properties associated with the particles detected in the HRS and electromagnetic calorimeter. The remainder of this chapter is concerned with combining this information to identify the RCS events of interest based upon the kinematic correlation between the scattered photon and recoil proton. The momentum, direction and reaction vertex of the recoil proton, as reconstructed in the hadron analysis and shown in fig. 5.1, are used to calculate the scattered photon four-momentum, from which the expected photon hit position on the front face of the calorimeter can be 
determined. This kinematic analysis procedure is described fully in Appendix A. The key variables obtained in these calculations are the reconstructed incident energy, $E_{\text {in }}$ (eq. A.8), and the all-important $x$ and $y$ coordinates for the difference between expected and measured calorimeter position, $\delta x$ and $\delta y$ (eq. A.14). These variables, together with the coincidence time and calorimeter energy, are then used to select the RCS events.

To fully understand the analysis of the production data, we must identify the potential sources of background events and their expected contribution for the present experimental arrangement. In addition to real Compton scattering there are four distinct types of event:

1. $p\left(\gamma, \pi^{0} p\right)$ followed by $\pi^{0} \rightarrow \gamma \gamma$

By far the most dominant reaction is coherent $\pi^{0}$ photoproduction on the proton, where due to its low mass the four-momentum of the $\pi^{0}$ is almost identical to that of the Compton scattered photon. On a very short timescale, the pion decays into two photons which are emitted back-to-back in its rest frame. The Lorentz boost to the laboratory means that one of these photons will have an energy between $E_{\pi} / 2$ and $E_{\pi}$, and will be located within a cone of half-angle $m_{\pi} / 2$; while the other photon will have an energy less than $E_{\pi} / 2$ and will lie outside the cone. Due to the limited solid angle subtended by the calorimeter at large distances from the target only the higher energy of the two photons will normally be detected.

2. $p\left(e, e^{\prime} p\right)$

The second most prevalent process is elastic scattering of electrons in the mixed beam from target protons. As discussed in sec. 4.4.3, the introduction of the magnet between target and calorimeter allows separation between the $e p$ and RCS events, which would otherwise be indistinguishable.

3. $p(e, p \gamma) e^{\prime}$

A much smaller but certainly non-negligible background arises from elastic ep scattering followed immediately by collinear emission of a hard bremsstrahlung photon (labeled as ep $\gamma$ events). If the bremsstrahlung photon produced in this process carries almost all of the initial electron energy, it travels in the same direction and can not be discerned from the Compton scattered photons. Monte Carlo studies have shown that this process is significantly suppressed relative to the Compton process at large scattering angles. 


\begin{tabular}{|c|c|c|}
\hline & Mean & Range (FWHM) \\
\hline \hline Incident Photon Energy $\left(E_{\text {in }}\right)$ & ${ }^{\circ} 3.216 \mathrm{GeV}$ & $0.258 \mathrm{GeV}$ \\
\hline CM Scattering Angle $\left(\Theta_{c m}\right)$ & $117^{\circ}$ & $12^{\circ}$ \\
\hline Mandelstam s & $6.92(\mathrm{GeV} / \mathrm{c})^{2}$ & $0.50(\mathrm{GeV} / \mathrm{c})^{2}$ \\
\hline Mandelstam t & $-4.01(\mathrm{GeV} / \mathrm{c})^{2}$ & $0.42(\mathrm{GeV} / \mathrm{c})^{2}$ \\
\hline Mandelstam u & $-1.15(\mathrm{GeV} / \mathrm{c})^{2}$ & $0.22(\mathrm{GeV} / \mathrm{c})^{2}$ \\
\hline Calorimeter Distance $\left(D_{\text {calo }}^{\prime}\right)$ & $5.7 m$ & - \\
\hline Calorimeter Central Angle $\left(\Theta_{\text {calo }}\right)$ & $65^{\circ}$ & - \\
\hline HRS Central Angle $\left(\Theta_{H R S}\right)$ & $19.47^{\circ}$ & - \\
\hline Total Accumulated Charge & $15.6 \mathrm{C}$ & - \\
\hline
\end{tabular}

Table 5.1: Production data kinematics and experimental conditions.

4. $\geq 3$-body processes

Reactions such as $\pi^{0}$ production through intermediate nucleon resonances or $\eta$ photoproduction have cross sections which are comparable with the other processes in the experiment. However, the absence of a strong kinematic correlation between the protons and mesons in these processes means that the phase space is not strongly populated in the spectrometer acceptances, unlike the reactions described above. As an example, an $\eta$ meson decays to two photons with a branching ratio of $40 \%$, but the fact that its mass is four times larger than the pion means that its decay cone is sixteen times larger. Therefore, $\geq 3$-body reactions such as this will not contribute appreciably to the background in this experiment.

\subsubsection{Event Identification}

To enhance the selection of the RCS events over the background processes described above, a series of cuts must be applied to the data. These cuts involve the coincidence time, the calorimeter energy and the kinematically reconstructed energy of the incident photon/electron. Spectra for all these variables, with and without cuts applied, are shown in fig. 5.4. The corresponding kinematic variables for the production data are shown in table 5.1.

\section{Coincidence Time}

Rejecting events which do not have a tight timing relation between the hadron and photon triggers is an important first step in cleaning up the data. For each trigger 

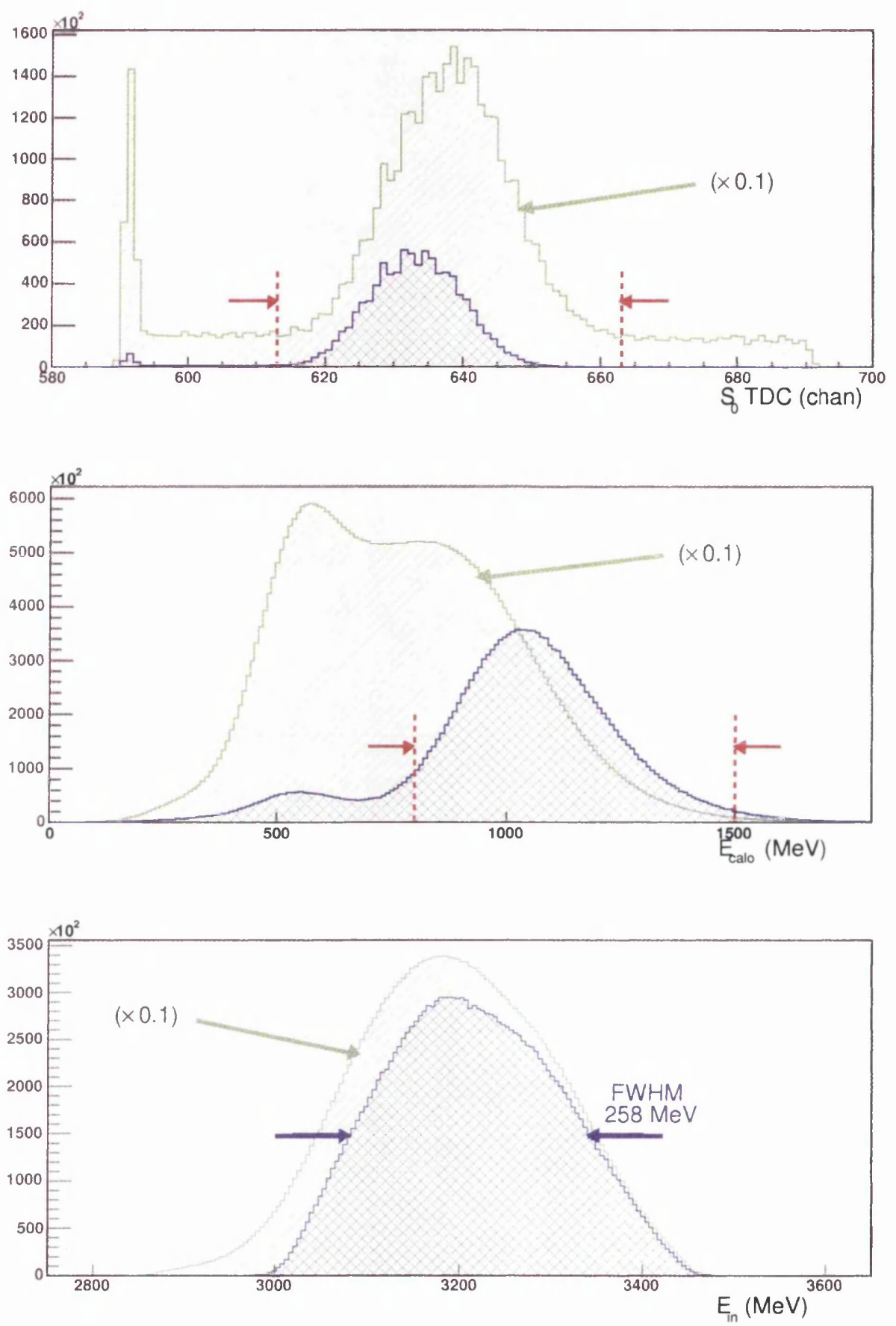

Figure 5.4: Spectra for three key variables: hadron trigger TDC (top), calorimeter energy (middle) and reconstructed incident energy (bottom). All plots are shown with (blue) and without (green) cuts to select RCS events. For transparency the data without cuts have been scaled by 0.1. 
type (T1, T5 and T7) a TDC module records the time when the corresponding trigger is received. The top plot in fig. 5.4 shows the raw TDC spectrum for the hadron (T7) trigger. The peak at the centre of this distribution corresponds to the region where there are good coincident events, whereas events out with this region are due to false coincidences, for which a proton produces a signal in the HRS but there is no accompanying, correlated signal in the calorimeter. During the present measurement three changes were made to the coincidence electronics, which means that three separate sets of TDC cuts had to be identified ${ }^{1}$ :

\begin{tabular}{|c|c|}
\hline Run Number & TDC Window (channels) \\
\hline \hline $2725-2759$ & $624-674$ \\
\hline $2760-3202$ & $613-663$ \\
\hline $3203-3462$ & $598-648$ \\
\hline
\end{tabular}

\section{Calorimeter Energy}

While no explicit energy dependence is expected from the $\geq 3$-body processes, all of the kinematically correlated processes can be characterised by their dependence on the detected photon/electron energy $\left(E_{\text {calo }}\right)$. This makes the total energy deposited in an electromagnetic shower a useful variable. The distribution of the calorimeter energy is shown in the middle plot in fig. 5.4:

- The RCS and ep events are concentrated in the upper half of the spectrum.

- Photo-pion events are spread out over the full range, although concentrated in the lower half.

- The $e p \gamma$ events display the characteristic bremsstrahlung behaviour described in sec. 4.2.2: the number of photons is largest at low energy and falls off as $1 / E_{\text {calo }}$.

Therefore, the cuts chosen to enhance the Compton events were chosen to be:

$$
800 \mathrm{MeV}<E_{\text {calo }}<1600 \mathrm{MeV}
$$

\footnotetext{
${ }^{1}$ A common width of 25 ns ( 50 channels) was chosen.
} 


\section{Reconstructed Incident Energy}

The four-momentum of the detected proton in a two-body process uniquely defines the incident electron/photon energy $\left(E_{i n}\right)$. The range of this incident energy is predetermined by the position and acceptance of both photon and hadron spectrometers (sec. 4.2.2), and is shown on the bottom plot in fig. 5.4. Cuts have been placed on this quantity to ensure events are well within the acceptance:

$$
3070 \mathrm{MeV}<E_{\text {in }}<3380 \mathrm{MeV}
$$

\section{$\delta x-\delta y$ Coordinates}

The most useful quantities for identifying the Compton events are the coordinates of the difference between the measured and predicted calorimeter hit position $(\delta x$ and $\delta y)$. The 2-D distribution of these variables, after application of the aforementioned cuts on coincidence time, calorimeter energy and reconstructed incident energy, is shown in the top plot of fig. 5.5. Three regions can clearly be identified:

1. A continuous background distribution, resulting predominantly from the detection of the higher energy photons from $\pi^{0}$ decay. These background events cover the whole of the $\delta x$ and $\delta y$ range and the distribution reaches a maximum at $\delta x \simeq 0, \delta y \simeq 0$. The bottom left plot in fig. 5.5 shows that the relationship between photon energy and opening angle in the pion decay gives rise to a strong $\mathrm{V}$-shape correlation between $\delta y$ and $E_{\text {calo }}$ (the same is true for $\delta x)$.

2. Two distinct peaks can be seen on top of this background region. The first of these, also centered at $\delta x \simeq 0, \delta y \simeq 0$, corresponds to real Compton scattering events. The width of this distribution is determined by the combined angular resolution of the HRS and calorimeter.

3. The peak located at $\delta x \simeq-20 \mathrm{~cm}, \delta y \simeq 0$ arises from elastic ep scattering events where the scattered electron has been deflected in the RCS magnet. It is clear from the $\delta x$ projection (and fit) shown in the bottom right of fig. 5.5 that the RCS and ep peaks are sufficiently separated to distinguish between the two. 


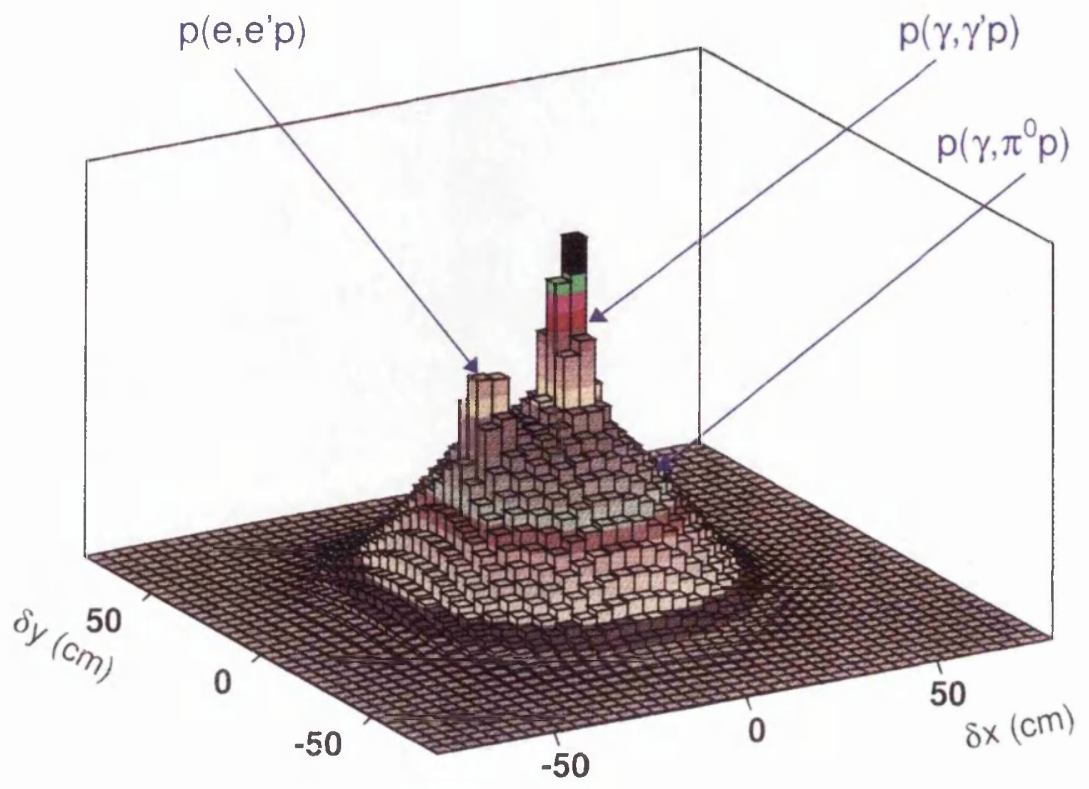

$\delta y(\mathrm{~cm})$
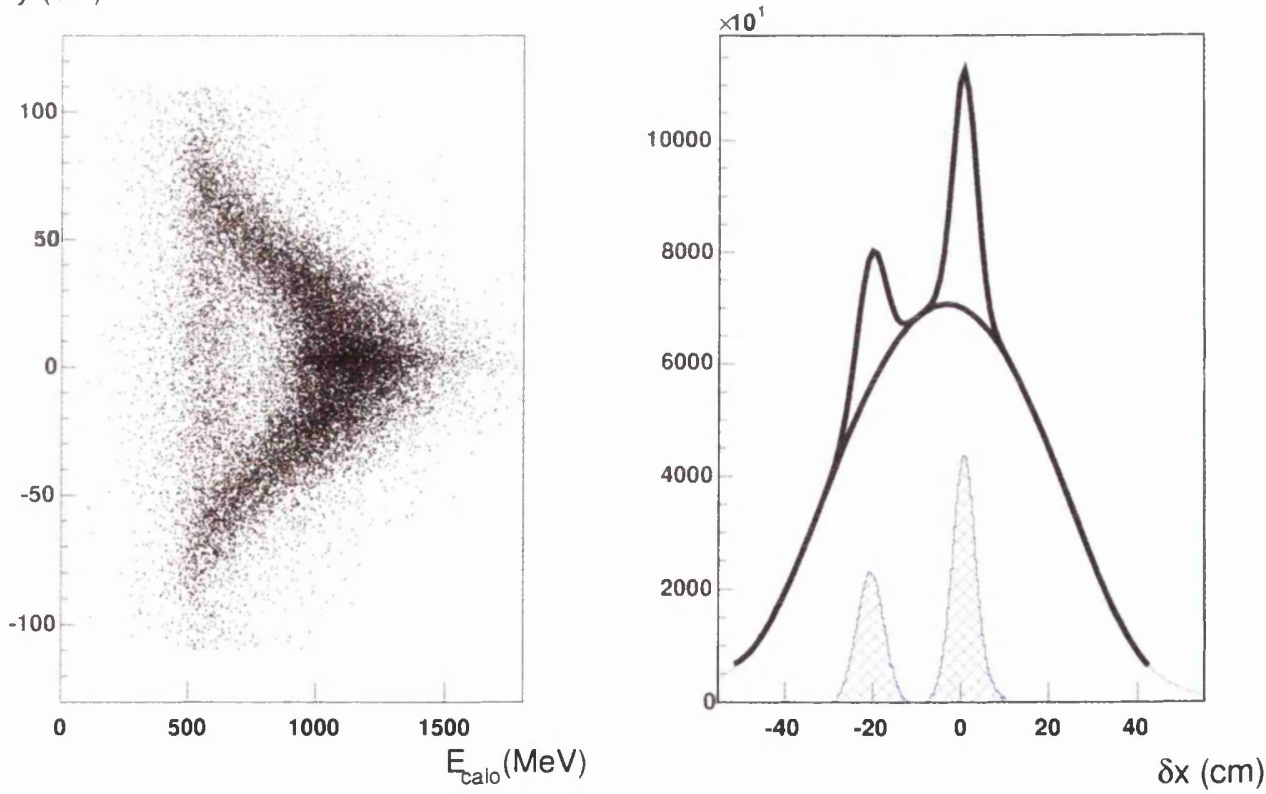

Figure 5.5: Event identification via the difference in measured and predicted calorimeter hit position as described in the text. Top: $\delta x$ vs $\delta y$; Bottom left: $\delta y$ vs $E_{\text {calo; }}$ Bottom right: projection onto $\delta x$ axis and fits to the background, $R C S$ and ep distributions. 


\subsubsection{Background Correction}

Photons which are detected at the centre of the $\delta x-\delta y$ distribution may have arisen from RCS, photo-pion or $e p \gamma$ processes. Since there is no way to further separate these events, the determination of the RCS polarisation observables involves calculating the polarisation for the two background processes independently, and applying a correction for the dilution caused by these events. Therefore, one needs to know the respective ratios for the number of background to RCS events.

\section{Post-Radiator Bremsstrahlung Events}

Monte-Carlo studies conducted at the preparation stage of the experiment [58] have found that the ratio $N_{e p \gamma} / N_{r c s}$ is expected to be less than $5 \%$ for the present kinematics. Even so, with the electron transferring almost all its polarisation to the hard bremsstrahlung photon in this process (see Appendix B) the polarisation contamination could still be significant. For a more accurate estimate of this ratio, a limited amount of elastic ep data was taken at the endpoint kinematics with the RCS magnet on. This provides an empirical measure of the ratio $N_{e p \gamma} / N_{e p}$, from which the equivalent ratio to RCS events can easily be extracted. The $\delta x$ spectrum for this data is shown in fig. 5.6 with cuts to remove background. The events concentrated around $\delta x=-20 \mathrm{~cm}$ are the elastic ep scattering events, while those around zero correspond to the $e p \gamma$ events. An approximate estimate of the ratio of events in these regions gives $N_{e p \gamma} / N_{e p} \simeq 0.04$, which in turn gives the ratio of post-radiator bremsstrahlung to RCS events:

$$
N_{e p \gamma} / N_{r c s} \simeq 0.024
$$

\section{$\pi^{0}$ Background}

To calculate the ratio $N_{\pi} / N_{r c s}$ for events in a particular $\delta x-\delta y$ region, we require a parameterisation of the shapes of both the RCS and background distributions in fig. 5.5. A naive first approximation for obtaining this ratio would be to fit projections onto the $\delta x$ and $\delta y$ axes as in the bottom right plot in fig. 5.5. However, such a procedure would not afford the optimum selection since any $\delta x-\delta y$ region defined by appropriate cuts would be rectangular in shape; the RCS distri- 


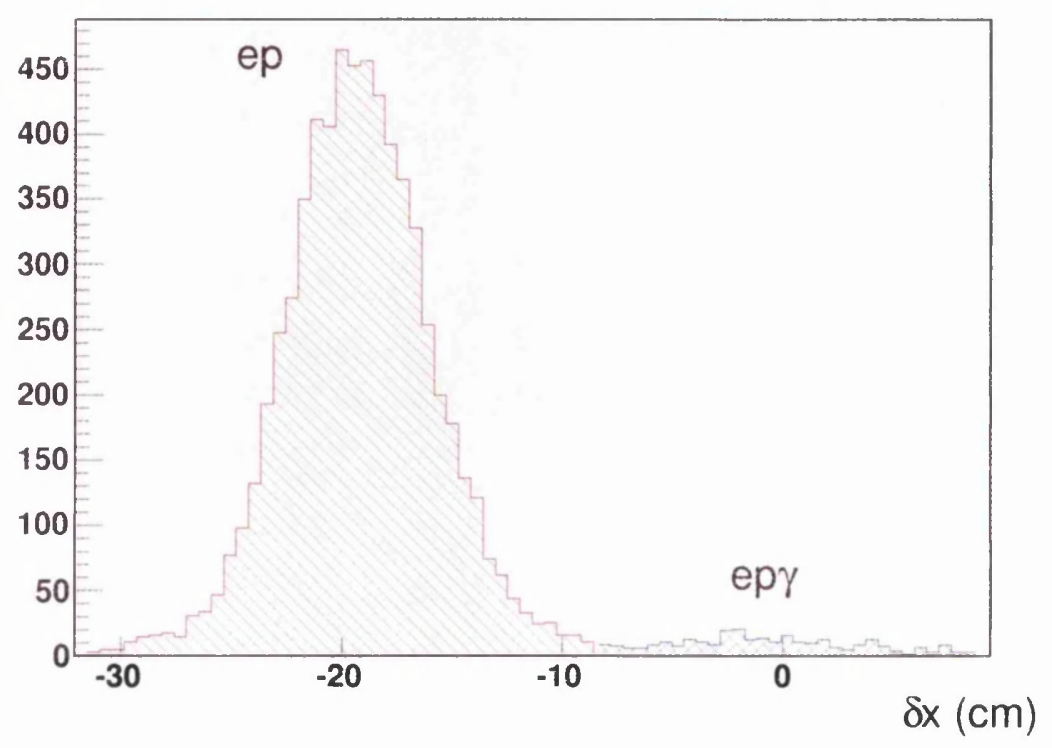

Figure 5.6: $\delta x$ spectrum for calibration data taken with the deflection magnet on. The elastic ep events dominate and are located around $\delta x=-20$, whereas the potential ep $\gamma$ events are centered around $\delta x=0$.

bution clearly exhibits elliptical symmetry. Nonetheless, this type of projection fitting hints at a more self-consistent parameterisation. Because the production data has such large statistics very precise 2-dimensional parameterisations of the background and RCS distributions can be found by fitting slices of the $\delta x-\delta y$ distribution.

This is best illustrated by considering the histograms in fig. 5.7, which have been produced by taking 100 slices in $\delta x$ and fitting the $\delta y$ projections. These plots show the fitted data for a selection of slices: a 2 nd order polynomial has been used to fit the background contribution, while the RCS events on top have been fitted with a Gaussian function. By obtaining the integral of these functions over a specified region for each slice, a sum over all slices gives the ratio of background to RCS events. By using the equation of an ellipse to define the cuts for each individual slice, this ratio has been calculated for three elliptical regions around the RCS peak:

\begin{tabular}{|c|c|}
\hline ellipse & $N_{\pi} / N_{r c s}$ \\
\hline \hline $1 \sigma$ & 1.11 \\
\hline $2 \sigma$ & 2.08 \\
\hline $3 \sigma$ & 4.08 \\
\hline
\end{tabular}



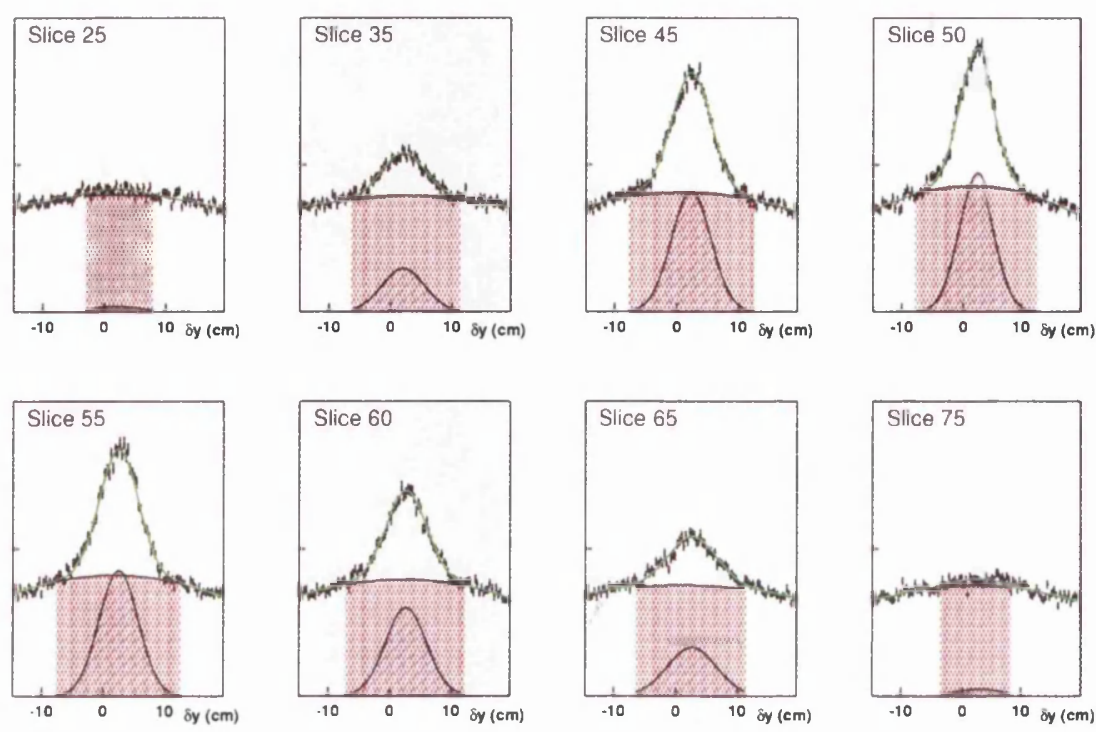

Figure 5.7: A selection of $\delta y$ projection fits for a procedure involving taking 100 slices in $\delta x$. The red shaded area highlights the number of background events within the chosen elliptical cut, the blue area shows the corresponding number of RCS events.

\subsection{Calibration Data}

The endpoint data was taken for calibration of the FPP analysing power. The procedure for doing so is described in the following chapter and requires a clean selection of elastic ep events. Identification of events which contain an elastically scattered electron and recoil proton in the calibration data is a much simpler task than identifying the Compton events in the production data. This is because the absence of the bremsstrahlung radiator means that background processes are far less prevalent. In fact, only small backgrounds from pion electroprduction and events which scatter and lose energy through bremsstrahlung are present in the calibration data. The kinematic variables associated with these runs can be found in tab. 5.2.

One does not learn much from examination of the calorimeter energy and coincidence timing spectra, and the cuts are the same as in the previous section. The reconstructed incident energy and $\delta x-\delta y$ spectra, on the other hand, are very different from the previous case, and are shown in fig. 5.8. The events in the $E_{\text {in }}$ spectrum (bottom left) are concentrated in a peak at the electron beam energy, with a radiative tail to the left of this peak produced by bremsstrahlung 


\begin{tabular}{|c|c|}
\hline Electron Beam Energy & $3.471 \mathrm{GeV}$ \\
\hline Four Momentum Transfer $Q^{2}$ & $4.06(\mathrm{GeV} / \mathrm{c})^{2}$ \\
\hline Calorimeter Distance $\left(D_{\text {calo }}^{\prime}\right)$ & $6.2 \mathrm{~m}$ \\
\hline Calorimeter Central Angle $\left(\Theta_{\text {calo }}\right)$ & $57^{\circ}$ \\
\hline HRS Central Angle $\left(\Theta_{\text {calo }}\right)$ & $21.36^{\circ}$ \\
\hline Total Accumulated Charge & $3.4 \mathrm{C}$ \\
\hline
\end{tabular}

Table 5.2: Kinematic variables for the FPP calibration data.

processes in the target. By making a cut on this elastic peak of:

$$
3450 \mathrm{MeV}<E_{\text {in }}<3500 \mathrm{MeV}
$$

only the events centered at $\delta x=0$ on the bottom right plot remain. These correspond to the events from the elastic $e p$ reaction of interest. 

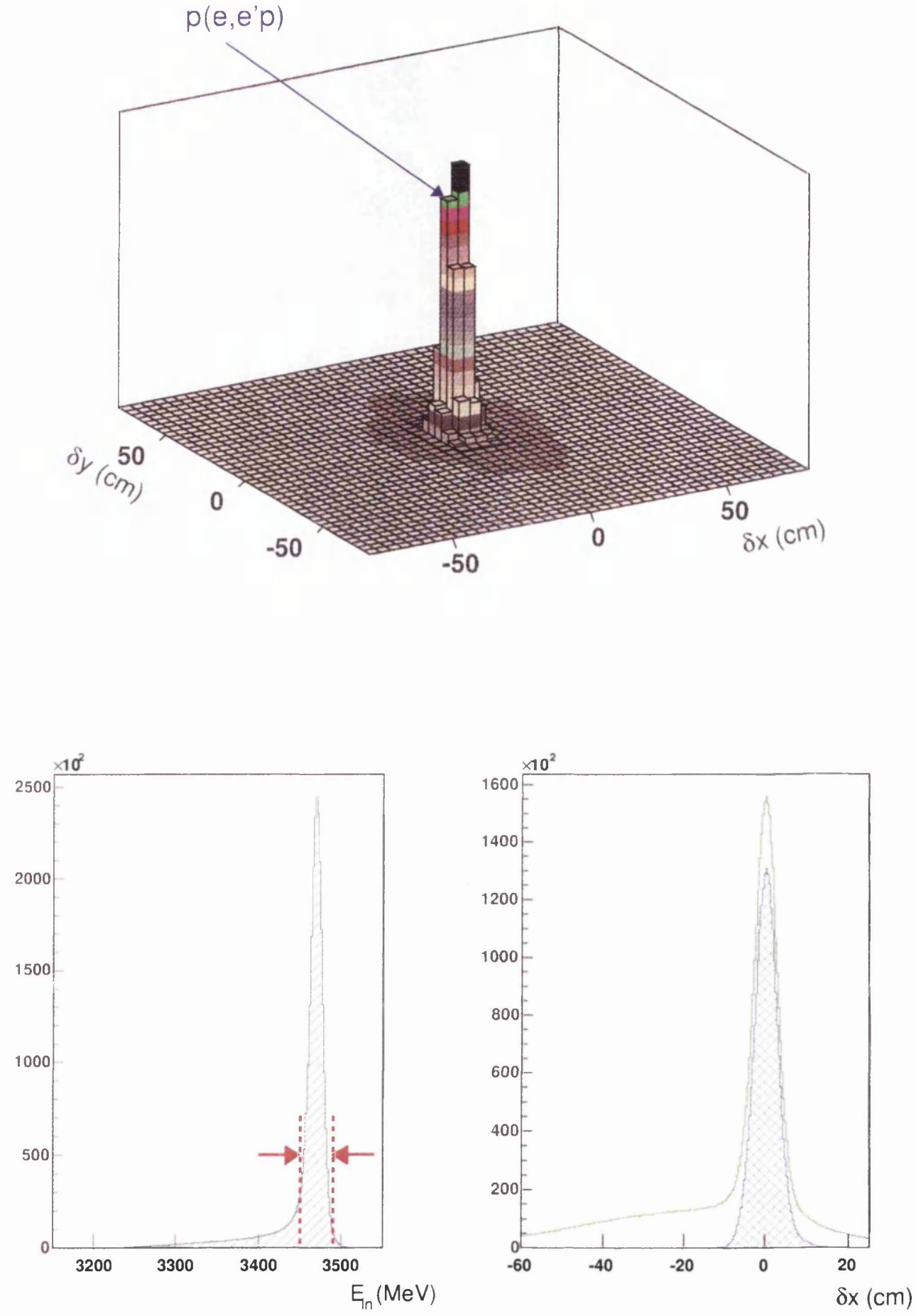

Figure 5.8: Demonstration of the event indentification procedure for calibration data. Top: $\delta x$ vs $\delta y ;$ Bottom left: reconstructed incident energy; Bottom right: projection onto $\delta x$ axis with (blue) and without (green) a cut on $E_{i n}$. 


\section{Chapter 6}

\section{Data Analysis: Polarisation Observables}

Once a particular set of events have been selected a general FPP analysis procedure is available for extracting the corresponding proton polarisation observables. The polarisation of a sample of protons which undergo secondary scattering via the strong interaction in the massive $\mathrm{CH}_{2}$ and carbon analysers located at the HRS focal plane gives rise to azimuthal asymmetries. These asymmetries allow access to the focal plane polarisation, which must be related to the primary polarisation of interest in a particular frame of reference at the target. To do so, one must be able to account for the precession of the proton spin vector in the HRS magnetic elements. Finally, to obtain physically meaningful polarisation components the FPP analysing power has to be measured in an independent experimental procedure using elastic $e p$ scattering data. Since the new double analyser FPP effectively provides two independent measurements of the same physical quantities, the final results are obtained by taking the average of the two. This chapter provides a description of the general analysis techniques which are identical for both analysers.

\subsection{Extraction of Focal Plane Asymmetries}

The FPP can be used to simultaneously measure the two recoil proton polarisation components normal to the direction of the proton's momentum at the spectrometer focal plane by determining asymmetries in the azimuthal angular distributions after scattering in one of the analysers. To form these asymmetries one must measure the proton's trajectories before and after each of the analysers, 

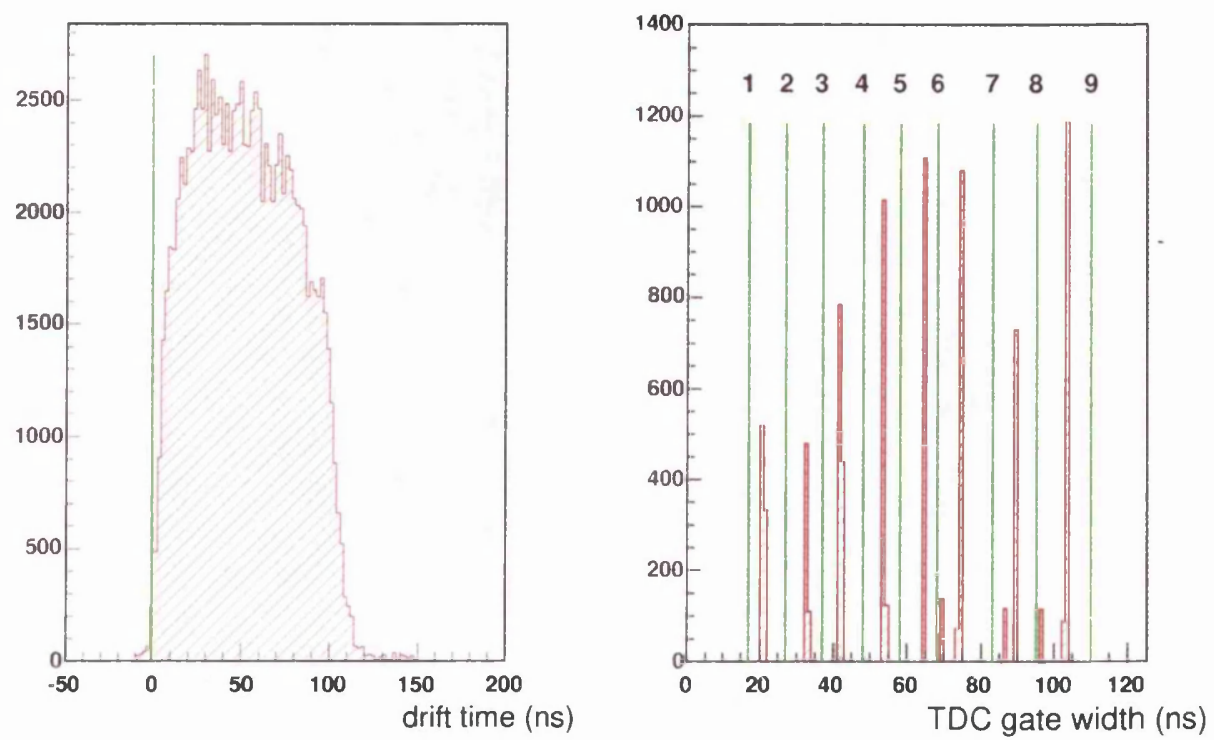

Figure 6.1: Left: typical drift time (LE) spectrum after calibration of the $t_{0}$ for this particular channel (shown in green). Right: distribution of the TDC gate widths (LE-TE time), with the individual demultiplex gates (green) used to extract which wire in a group of eight has fired.

from which scattering observables must be reconstructed.

\subsubsection{Straw Chamber Tracking}

The general principles behind the calibration of tracking chambers and track reconstruction algorithms were first discussed with regard to the VDC's in sec. 5.2.2. The procedure essentially involves the measurement of the intercept and slope of a particular trajectory from identifying which wire has been hit and calculating the drift distance from the wire. In addition to being used to reconstruct the proton trajectory at the target, the VDC's were also used in combination with the FPP straw chambers (described in sec. 4.3.3) to measure the angles through which protons scatter in the FPP analysers. The analysis specific to the straw chamber devices will be discussed in this section. Tracks are determined independently for the front and rear straw chambers, although the analysis procedure used for both is exactly the same

Calibration of the straw chambers requires the raw data from a straw hit, which includes the wire group (i.e. a particular electronics readout board), together with the leading-edge (LE) and trailing-edge (TE) times measured in the 
corresponding TDC channel. Three separate calibration procedures are then implemented, with coefficients stored in databases which are read by the analysis software at a later stage:

1. Each TDC channel has associated with it a reference time $\left(t_{0}\right)$ which properly aligns the zero of the drift time spectrum with the actual time of the particle hit. These $t_{0}$ values have been determined for each channel using an automated software procedure. A typical drift time spectrum after $t_{0}$ calibration can be seen on the left-hand side of fig 6.1.

2. The demultiplex gates for each individual wiregroup must be determined from the data. As described in sec 4.3.3, the signals from each straw are multiplexed in groups of eight, and the width of the readout logic pulse is related to which of the wires was actually hit. Therefore, appropriate demultiplex gates must be identified in the LE-TE time spectrum to relate gate width to wire hit. An example of such a distribution is shown on the right-hand side of fig 6.1, along with the corresponding demultiplex cuts.

3. The non-uniform nature of the electric field around the sense wire at the centre of the particular straw means that the drift velocity is not constant. In fact, there is a non-linear relationship between drift time and drift distance. For each plane in the straw chambers, a fifth-order polynomial fit has been performed to relate the drift distance to the integrated drift time.

After these straw chamber calibration procedures have been performed, it is possible to identify proton trajectories and determine their physical properties. The procedure for doing so is relatively straightforward and involves finding clusters of adjacent straw hits. The minimum requirement for a hit pattern to be accepted as a good track is a total of three clusters in two adjacent chambers. A straight-line fit, based upon a $\chi^{2}$ minimisation algorithm, is then used to find the best track from all available clusters for a given event. A rough estimate is first obtained from the wire hit position, and a weight is assigned to each track proportional to the number of hits associated with it. The procedure is then refined by including the drift distance information, and finding whether a left or right drift distance gives the best fit. 


\subsubsection{Straw Chamber Alignment}

Before using the track parameters to calculate the proton scattering angles, the respective chambers must be mutually aligned in the software. While the physical alignment of the FPP straw chambers is relatively precise, it is certainly not at the level required for precise scattering angle reconstruction. The FPP reconstruction software works on the assumption that the chambers are centered on the spectrometer $z$-axis and orientated perfectly parallel to each other. Any deviations from this will result in a significant contribution to the instrumental, or false, asymmetries.

A software procedure is therefore used to calibrate the chamber positions, with the desired accuracy of $\simeq 100 \mu \mathrm{m}$. In the time that the FPP has been operational in Hall $\mathrm{A}$, three different methods have been employed for this purpose. The common feature in all these procedures is the comparison of the front and rear trajectories relative to the corresponding tracks determined by the pair of VDC's. The VDC's are taken as a reference and all other chambers must be orientated according to them:

$$
\left(\begin{array}{l}
x \\
y \\
\theta \\
\phi
\end{array}\right)_{V D C}=\left(\begin{array}{l}
x \\
y \\
\theta \\
\phi
\end{array}\right)_{F P P_{\text {front }}}^{\text {aligned }} \cdot\left(\begin{array}{l}
x \\
y \\
\theta \\
\phi
\end{array}\right)_{F P P_{\text {rear }}}^{\text {aligned }} .
$$

The alignment procedure is then very simple: for each track parameter $(x, y, \theta$ and $\phi$ ), for both front and rear chambers separately, the difference between the measured and reference tracks must be minimised. Ideally, to keep multiple (or Coulomb) scattering to a minimum and ensure the trajectories are not significantly altered, there should be no material between the chambers in the alignment data. In practice, however, it is more than sufficient to take "straight-through" data with only the analyser blocks removed: other focal plane detectors, such as the scintillator planes, do not significantly alter the trajectories of the high momentum protons of interest.

The most recent and most effective of the alignment techniques was used for FPP calibration for the RCS experiment [84]: The most important question concerning this technique is how to modify the track variables in such a way that the difference between the measured and reference tracks is minimised. The 
solution involves finding a function for each track parameter such that:

$$
\left(\begin{array}{c}
x \\
y \\
\theta \\
\phi
\end{array}\right)_{F P P_{\text {rear }}}^{\text {aligned }}=\left(\begin{array}{c}
x \\
y \\
\theta \\
\phi
\end{array}\right)_{F P P_{\text {rear }}}^{\text {raw }}+\left(\begin{array}{c}
f_{\text {rear }}^{x}\left(\text { track }_{\text {raw }}\right) \\
f_{\text {rear }}^{y}\left(\text { track }_{\text {raw }}\right) \\
f_{\text {rear }}^{\theta}\left(\text { track }_{\text {raw }}\right) \\
f_{\text {rear }}^{\phi}\left(\text { track }_{\text {raw }}\right)
\end{array}\right) .
$$

The current choice for $f\left(\operatorname{track}_{\text {raw }}\right)$ is dependent only on $x$ and $y$, and has the general form:

$$
f\left(\operatorname{track}_{\text {Raw }}\right)=a x^{2}+b y^{2}+c x y+d x+e y+f
$$

There is one such function for each of the four track variables, for both front and rear chambers, leading to a total of 48 unknown coefficients. The minimisation is performed by constructing a set of linear equations with these coefficients as unknowns and solving the set by means of LU (Lower-Upper) decomposition. This method is one of the more powerful techniques. for solving linear algebraic equations, and involves breaking up the set of equations into two smaller triangular sets. (see ref. [85] for more details).

This technique was applied to the RCS "straight-through" calibration data, taken specifically for this purpose at the central HRS proton momentum of $4.3 \mathrm{GeV} / \mathrm{c}$. The distributions of the difference between the measured and reference tracks, after alignment, for front and rear FPP chambers can be seen in fig. 6.2 $2^{1}$. It is expected that the distribution for each variable is approximately Gaussian and centered at $\approx 0$, with a value of sigma that is directly related to the position resolution of the VDC's, the position resolution of the FPP straw chambers and the multiple scattering between them, the latter being the most dominant contribution. An estimate of typical values of the angular spread due to multiple scattering can be obtained by considering the compositions and thicknesses of the materials in the focal plane (scintillators, aerogel and the chambers themselves) through the formula:

$$
\Delta \Theta=\frac{13.6 \mathrm{MeV}}{\beta c p} Z \sqrt{\frac{x}{X_{0}}}\left[1+0.038 \ln \left(\frac{x}{X_{0}}\right)\right]
$$

where $\Delta \Theta$ is the polar angular spread due to Coulomb scattering, $p$ and $\beta c$ are the

\footnotetext{
${ }^{1}$ Due to a difference in the definition of coordinate systems between the VDC's and FPP Chambers, $\theta_{F P P}$ is compared with $\phi_{V D C}$ and vice-versa.
} 

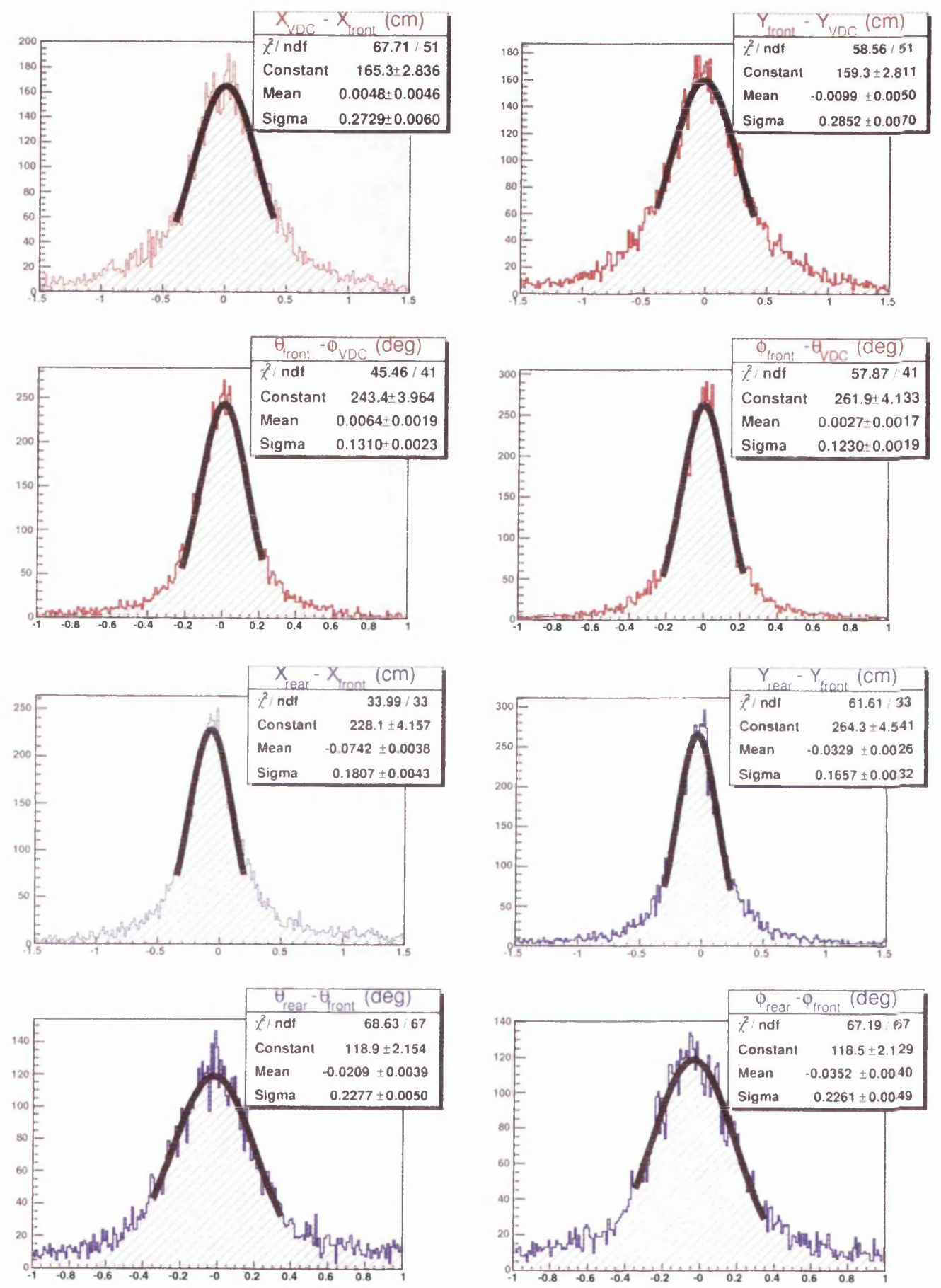

Figure 6.2: The post-alignment difference between the measured and reference trajectory variables - $x, y, \theta$ and $\phi$ - for front (top) and rear (bottom) straw chambers. 


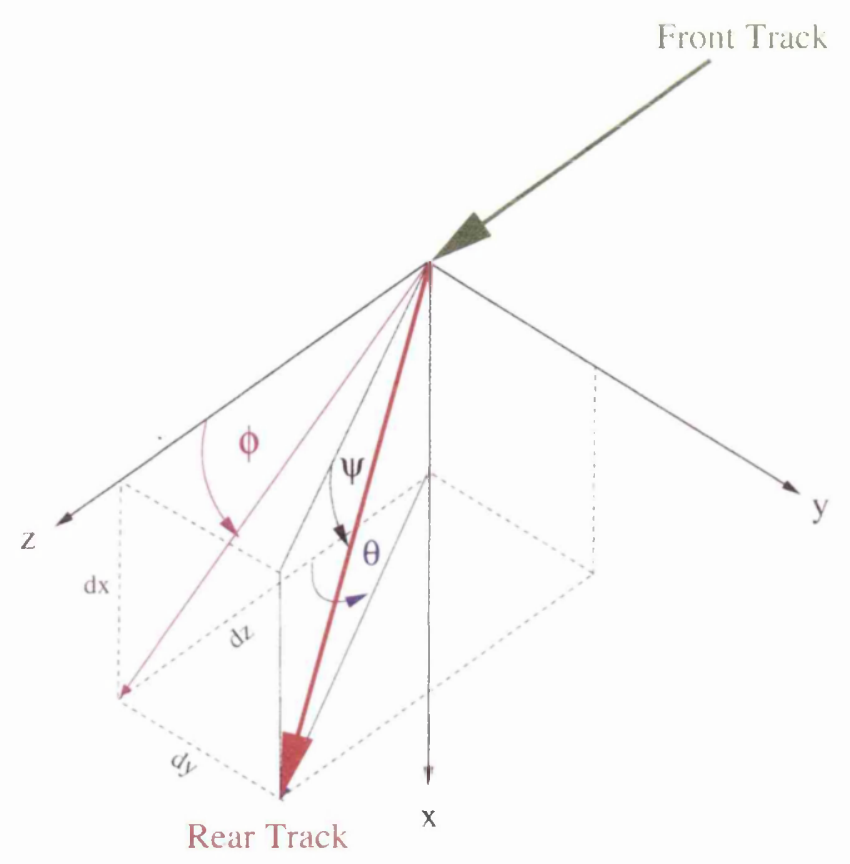

Figure 6.3: Definition of angles $(\theta, \phi, \psi)$ in FPP coordinate system, centered on the scattering vertex between the incoming (green) and outgoing (red) trajectories. The system is rotated so that the z-axis is aligned along the incoming trajectory.

momentum and velocity of the proton, and $Z$ and $x / X_{0}$ are the charge number and thickness in radiation lengths of the scattering medium.

It is testament to the success of the alignment procedure that all eight distributions in fig. 6.2 display the characteristics described above. For the front chambers, the distributions are all centered on zero with widths which are consistent with the expectations from Coulomb scattering effects. Although the rear distributions also look to be very well aligned, the widths of the angle differences distributions are larger than the calculations suggest. It was found that the reason for this was that one of the carbon doors was not removed during the alignment data taking period. It is clear from the figure that this has had a negligible impact on the effectiveness of the alignment process.

\subsubsection{Calculation of Scattering Observables}

\section{Scattering Angles}

With the front and rear track parameters $(x, y, \theta, \phi)$ as a starting point, calculation of the polar $(\vartheta)$ and azimuthal $(\varphi)$ scattering angles is a relatively simple exercise in geometry. 
In addition to the two previously defined trajectory angles, the angle between the front trajectory and the $y z$ plane must be defined:

$$
\tan \psi_{f}=\cos \theta_{f} \tan \phi_{f}
$$

The relationship between the angles $\theta, \phi$ and $\psi$ can be seen in fig. 6.4. This figure also shows that the scattering observables of interest are calculated in a coordinate system which has been rotated so that the incoming trajectory is aligned with the $z$-axis. This involves two rotations:

$$
\left(\begin{array}{l}
0 \\
0 \\
1
\end{array}\right)=\left(\begin{array}{ccc}
\cos \psi_{f} & 0 & -\sin \psi_{f} \\
0 & 1 & 0 \\
\sin \psi_{f} & 0 & \cos \psi_{f}
\end{array}\right)\left(\begin{array}{ccc}
1 & 0 & 0 \\
0 & \cos \theta_{f} & -\sin \theta_{f} \\
0 & \sin \theta_{f} & \cos \theta_{f}
\end{array}\right)\left(\begin{array}{c}
\hat{x_{f}} \\
\hat{y_{f}} \\
\hat{z_{f}}
\end{array}\right)
$$

The projection of the corresponding scattered trajectory $\left({\hat{x_{r}}}^{1},{\hat{y_{r}}}^{1},{\hat{z_{r}}}^{1}\right)$ is determined by applying the same combined rotation to the scattered track coordinates:

$$
\left(\begin{array}{c}
{\hat{x_{r}}}^{1} \\
{\hat{y_{r}}}^{1} \\
{\hat{z_{r}}}^{1}
\end{array}\right)=\left(\begin{array}{ccc}
\cos \psi_{f} & 0 & -\sin \psi_{f} \\
0 & 1 & 0 \\
\sin \psi_{f} & 0 & \cos \psi_{f}
\end{array}\right)\left(\begin{array}{ccc}
1 & 0 & 0 \\
0 & \cos \theta_{f} & -\sin \theta_{f} \\
0 & \sin \theta_{f} & \cos \theta_{f}
\end{array}\right)\left(\begin{array}{c}
\hat{x_{r}} \\
\hat{y_{r}} \\
\hat{z_{r}}
\end{array}\right) \text {. }
$$

The polar and azimuthal angles are then calculated in terms of this projection:

$$
\begin{aligned}
\vartheta & =\arctan \frac{\hat{r}}{\hat{z_{r}}}, \\
\varphi & =\arctan \frac{{\hat{x_{r}}}^{1}}{{\hat{y_{r}}}^{1}} \\
\hat{r} & =\sqrt{\left({\hat{x_{r}}}^{1}\right)^{2}+\left(\hat{y}_{r}\right)^{2}} .
\end{aligned}
$$

Typical plots of the distribution of polar scattering angles from the $\mathrm{CH}_{2}$ and carbon analysers are shown in fig. 6.4. The large peak on both plots at small values of $\vartheta$ arise as a result of protons which undergo Coulomb scattering in the particular analyser. Cuts are usually placed to select regions corresponding to strong nucleon scattering within the analysers, in kinematics where a significant analysing power is obtained. A full discussion of the cuts placed on the polar scattering angle, and their significance in terms of the analysing power, is given below in sec. 6.4. Another observation which can be made is that the number of events which scatter at larger values of $\vartheta$, relative to the Coulomb peak, is much 

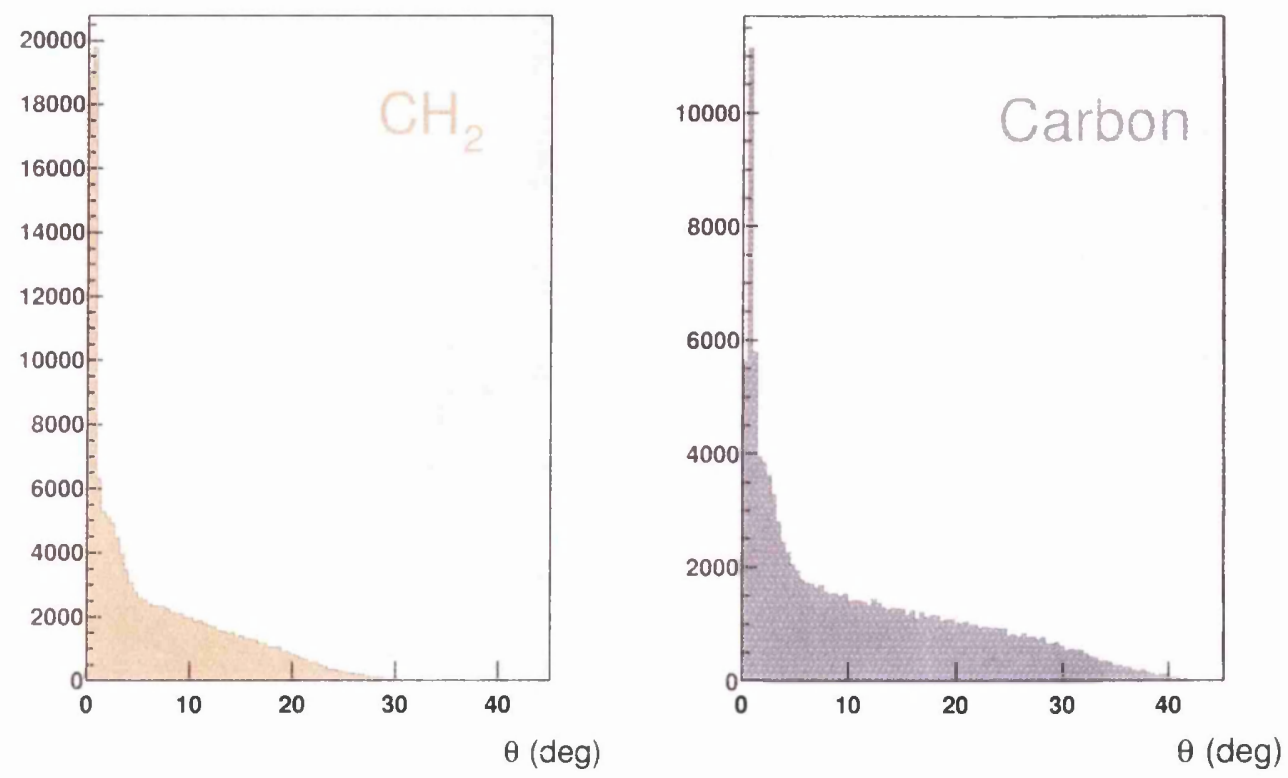

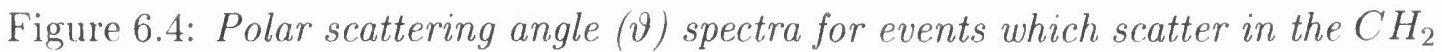
(left) and carbon (right) analysers.

smaller for the $\mathrm{CH}_{2}$ polarimeter than for the carbon. This can be attributed to the fact that the front FPP chambers, which detect the track scattered in the $\mathrm{CH}_{2}$ analyser, are much smaller than their rear counterparts (see sec. 4.3.3).

\section{Closest Approach Observables}

Two additional scattering observables which have proven to be indispensable in refining the selection of scattered events can be calculated:

1. The distance of closest approach between the incoming and scattered trajectories, $s_{\text {close }}$.

2. The $z$-coordinate of the midpoint of the line which joins the trajectories at closest approach, $z_{\text {close }}$.

If $A$ and $B$ are defined, such that:

$$
\begin{aligned}
& A=\left(x_{0}^{r}-x_{0}^{f}\right)+\left(\tan \phi^{r}-\tan \phi^{f}\right), \\
& B=\left(y_{0}^{r}-y_{0}^{f}\right)+\left(\tan \theta^{r}-\tan \theta^{f}\right),
\end{aligned}
$$


then

$$
\begin{aligned}
s_{\text {close }} & =\sqrt{\left(A z_{\text {close }}\right)^{2}+\left(B z_{\text {close }}\right)^{2}} \\
z_{\text {close }} & =\frac{A+B}{\left(\tan \phi^{r}-\tan \phi^{f}\right)^{2}+\left(\tan \theta^{r}-\tan \theta^{f}\right)^{2}}
\end{aligned}
$$

To demonstrate why these variables are useful typical distributions of $s_{\text {close }}$ and $z_{\text {close }}$ are shown for both analysers in fig. 6.5. Obviously, in an ideal polarimeter the incoming and scattered trajectories would intercept with $s_{\text {close }}=0$ and $z_{\text {close }}$ lying somewhere between the analyser $z$-axis boundaries. However, it is clear from these plots that the distributions are smeared somewhat by detector resolution effects or poor reconstruction, and by the fact that some events involve protons which physically scatter out with the analyser (from other detectors such as $\mathrm{S} 0$ ). Therefore, the polarimeter signal is enhanced by placing cuts to accept events with reasonably small values of $s_{\text {close }}$ and with $z_{\text {close }}$ within the physical analyser limits (as shown by the arrows in fig. 6.5):

1. $\mathrm{CH}_{2}: 220 \mathrm{~cm} \leq z_{\text {close }} \leq 264 \mathrm{~cm}$.

2. Carbon: $330 \mathrm{~cm} \leq z_{\text {close }} \leq 381 \mathrm{~cm}$.

\subsubsection{Azimuthal Asymmetries}

It is the spin-orbit coupling in the strong scattering of the recoil protons from nuclear targets within the FPP analysers which makes the current measurement possible. A sample of protons with their spins preferentially aligned in a particular direction gives rise to an azimuthal asymmetry in this scattering process. The component of the proton polarisation at the focal plane in the dispersive direction $\left(P_{x}^{f p p}\right)$ introduces a left-right asymmetry, while the non-dispersive component $\left(P_{y}^{f p p}\right)$ gives rise to an up-down asymmetry ${ }^{2}$. Therefore, the general expression for the azimuthal distribution of the scattered proton events for plus/minus helicity states is given by:

$$
\begin{aligned}
N^{ \pm}(\vartheta, \varphi)=N_{i n} \varepsilon(\vartheta) & {\left[1+\left(a_{0}-A_{y}(\vartheta) P_{x}^{f p p}\right) \cos \varphi\right.} \\
& +\left(b_{0}+A_{y}(\vartheta) P_{y}^{f p p}\right) \sin \varphi \\
& \left.+c_{0} \cos 2 \varphi+d_{0} \sin 2 \varphi\right]
\end{aligned}
$$

\footnotetext{
${ }^{2}$ There is no sensitivity to the polarisation component along the proton momentum.
} 


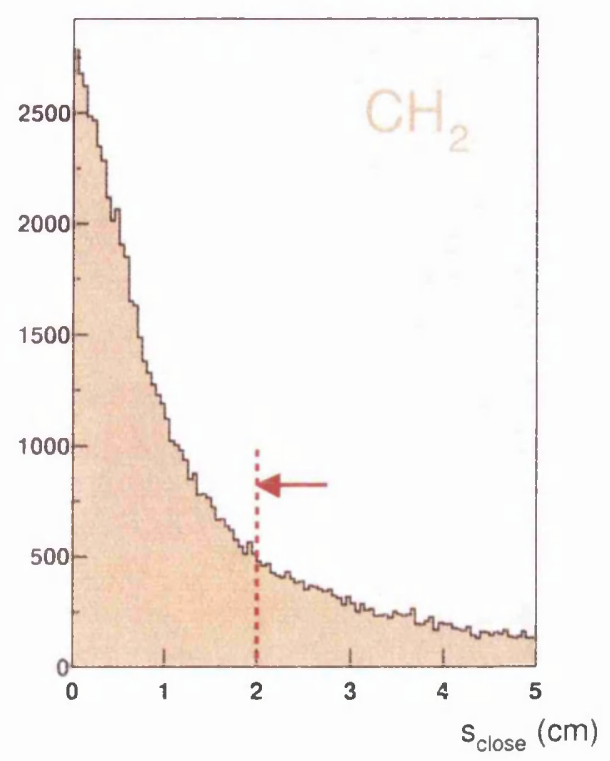

$\phi(\mathrm{deg})$

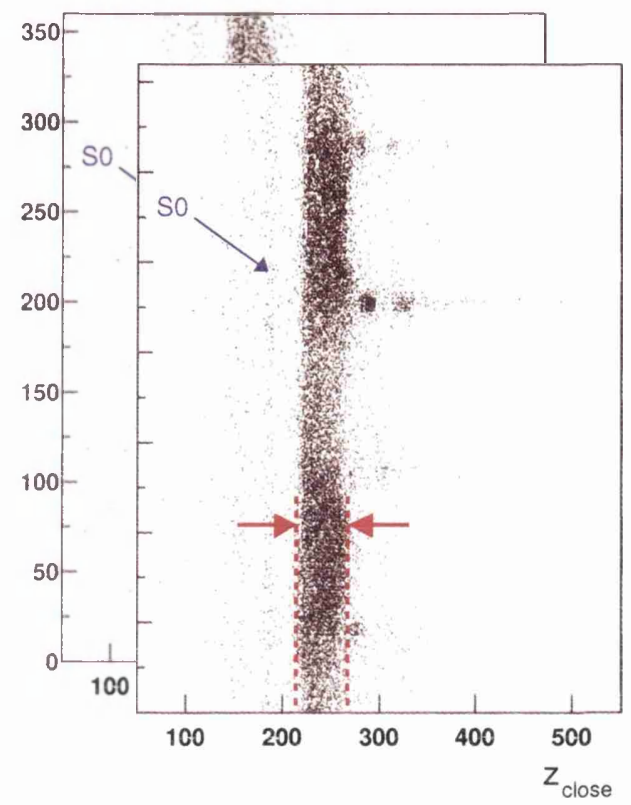

$\phi$ (deg)
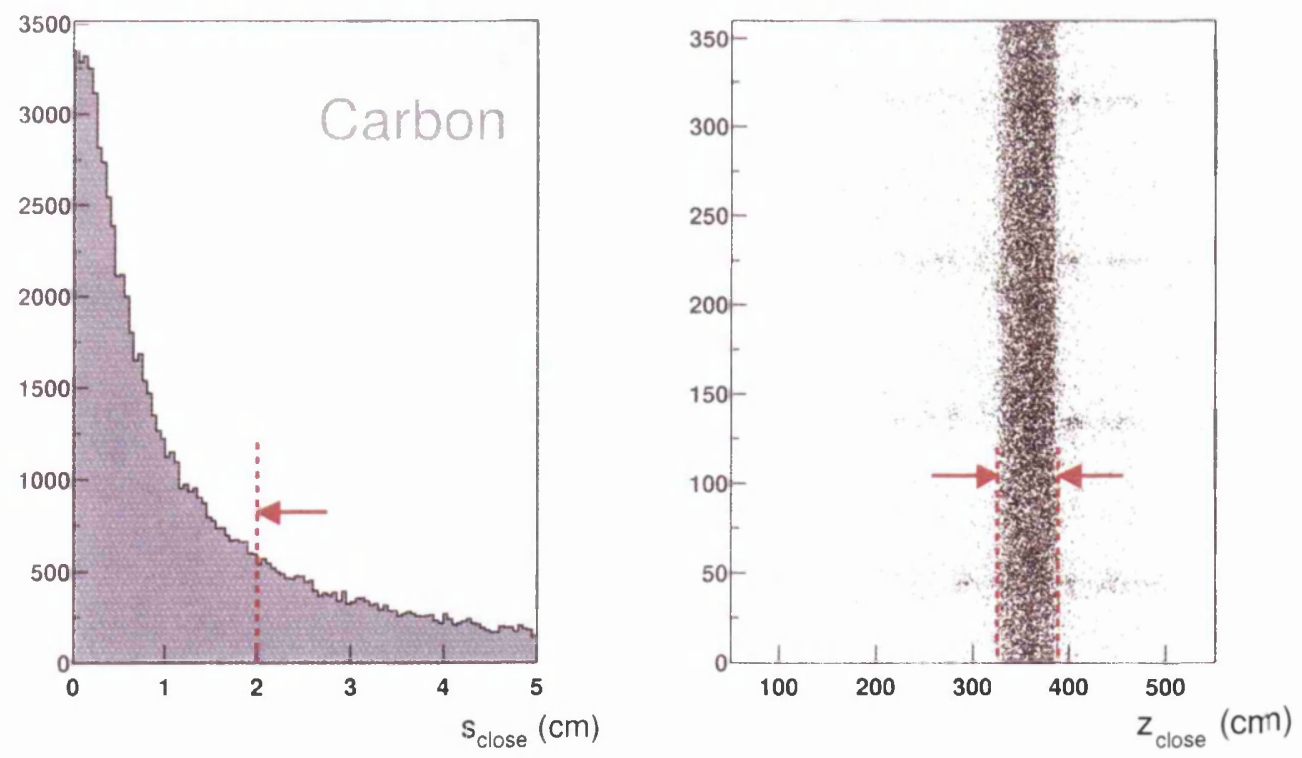

Figure 6.5: Distribution of the closest approach observables, $s_{\text {close }}$ and $z_{\text {closie }}$, for the $\mathrm{CH}_{2}$ (top) and carbon (bottom) analysers. The red arrows show the cuts applied to the data. 
where $N_{i n}$ is the number of incoming protons, $\varepsilon(\vartheta)$ is the efficiency of the polarimeter and $A_{y}(\vartheta)$ is the corresponding analysing power. This azimuthal distribution has contributions from $P_{x}^{f p p}$ and $P_{y}^{f p p}$, which are made up from induced and transferred polarisations in the primary reaction at the target, and from instrumental asymmetries parameterised by the variables $a_{0}, b_{0}, c_{0}$ and $d_{0}$.

In the present measurement, only the helicity-dependent polarisation transfer components are of interest. One can isolate these observables from the illstrumental asymmetries and induced polarisations, both of which are independent of the beam helicity, by taking the difference between the azimuthal distributions for plus and minus helicity states:

$$
\frac{1}{2}\left[\frac{N^{+}(\vartheta, \varphi)}{N_{i n}^{+}(\vartheta)}-\frac{N^{-}(\vartheta, \varphi)}{N_{i n}^{-}(\vartheta)}\right]=b(\vartheta) \sin \varphi-a(\vartheta) \cos \varphi
$$

where the normalisation by $N_{i n}^{ \pm}$avoids any false asymmetries which might arise because of differences between the number of incident events with plus and minus beam helicities. The magnitude of the beam-helicity asymmetry given in eq. 6.13 depends on the beam polarisation $(h)$ and the analysing power $\left(A_{y}\right)$, the latter being a measure of the strength of the spin-orbit coupling in the secondary scattering. Therefore, the focal plane polarisation transfer components can be obtained from the equations:

$$
\begin{aligned}
P_{x}^{f p p} & =\frac{a(\vartheta)}{h A_{y}(\vartheta)} \\
P_{y}^{f p p} & =\frac{b(\vartheta)}{h A_{y}(\vartheta)}
\end{aligned}
$$

The measurement of the analysing power is described in sec. 6.4 below, while the beam polarisation correction is considered in Appendix B.

The focal plane beam-helicity asymmetries measured in both polarimeters for events corresponding to the $\pi^{0}$ background reaction channel in the production data are shown in fig. 6.6. The data in these graphs have been divided into 60 equal bins of $\varphi$, and a sinusoidal fit of the type given in eq. 6.13 has been applied. The obvious conclusion to draw from this figure is that the magnitude of the $\mathrm{CH}_{2}$ asymmetry is both larger and has a smaller statistical uncertainty than the carbon asymmetry. Since they arise from precisely the same polarisation components, this implies that the analysing power of the $\mathrm{CH}_{2}$ must be larger than the carbon one. 

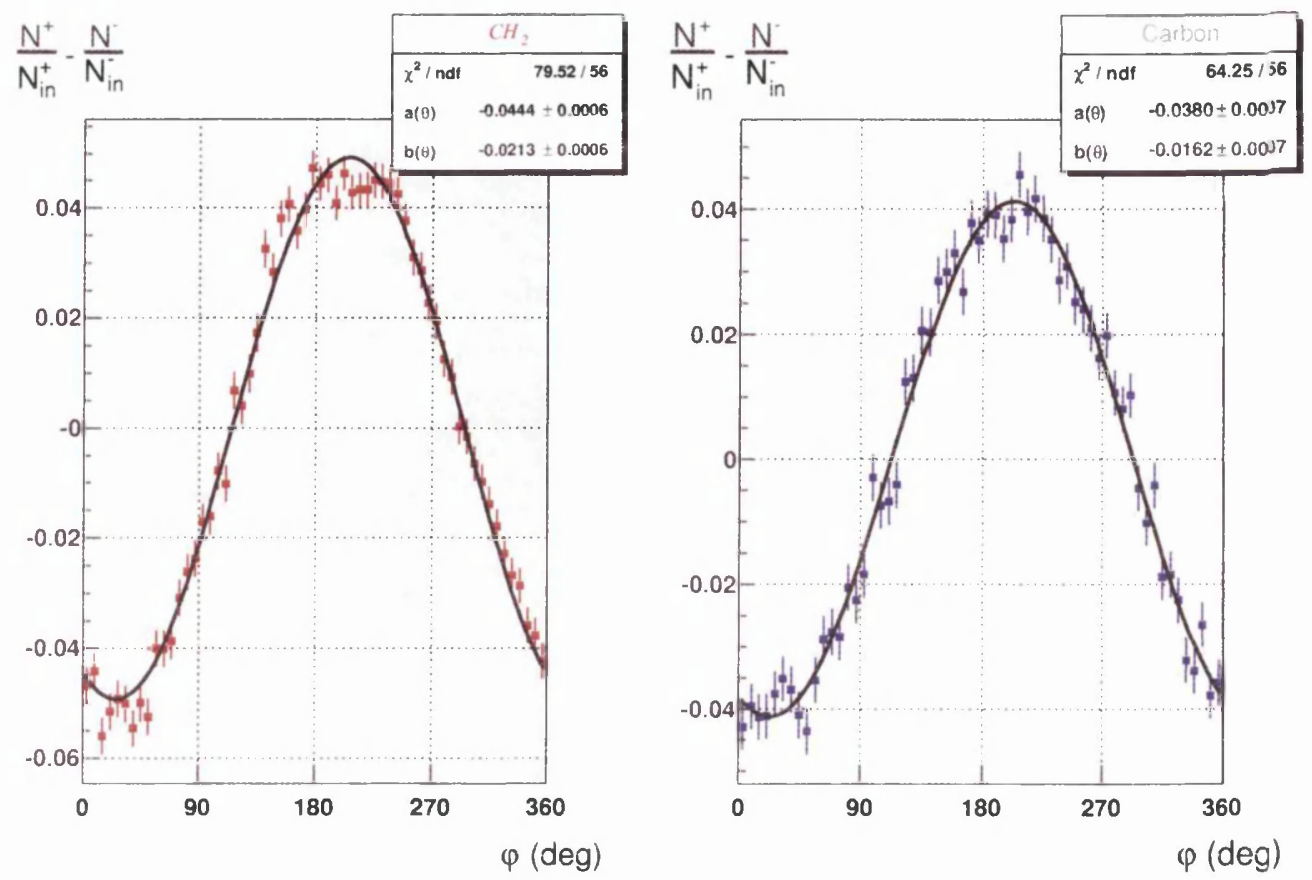

Figure 6.6: $\mathrm{CH}_{2}$ and carbon beam-helicity asymmetries for production events identified as $\pi^{0}$ background. The data are fitted by a asin $\varphi+b \cos \varphi$ function, the coefficients for which are shown in the top right legends.

It is by this method that the proton polarisation transfer terms at the target can be extracted. A discussion of the procedure is given in sec. 6.3 , but before that, there are two significant complications. Firstly, in the general case there will be both induced polarisation terms and instrumental asymmetries. Access to these is through a complementary Fourier analysis of the helicity-sum distributions. Secondly, the issue of relating the proton polarisations at the focal plane to the corresponding polarisations at the target is not straightforward. It leads to the complex problem of understanding spin precession in the HRS.

\section{False Asymmetry}

False (or instrumental) asymmetries arise from misalignment of the straw chambers of the FPP and from local efficiency variations in the chambers due to faulty wires and the like. To quantify these instrumental asymmetries a measurement of the focal plane asymmetry from unpolarised protons, which would ideally be perfectly flat, is required. Since an unpolarised proton sample is not available, the next best thing is to use the helicity-sum distribution, which in the absence of induced polarisation in the primary reaction is equivalent to an unpolarised 


\begin{tabular}{|c|c|c|c|}
\hline & $\mathrm{e} 99007$ & $\mathrm{e} 99114 \mathrm{CH}_{2}$ & $\mathrm{e} 99114$ carbon \\
\hline$a_{0}$ & $0.0160 \pm 0.007$ & $0.0084 \pm 0.0024$ & $-0.0553 \pm 0.0025$ \\
$b_{0}$ & $0.0019 \pm 0.007$ & $0.0037 \pm 0.0024$ & $0.0047 \pm 0.0025$ \\
$c_{0}$ & $-0.0069 \pm 0.007$ & $0.0064 \pm 0.0024$ & $0.0026 \pm 0.0025$ \\
$d_{0}$ & $0.0086 \pm 0.007$ & $0.0256 \pm 0.0024$ & $-0.0015 \pm 0.0025$ \\
\hline
\end{tabular}

Table 6.1: Four lowest false asymmerty amplitudes obtained from the ep calibration data, compared with those found in a single analyser FPP configuration in e9900\%.

one. In the one-photon exchange approximation of elastic ep scattering there is no induced polarisation in the proton (except from higher order effects which have been estimated at less than $1 \%$ ), which makes the calibration data ideal for extracting the instrumental asymmetries. Thus:

$$
\frac{1}{2}\left[\frac{N^{+}(\vartheta, \varphi)}{N_{i n}^{+}(\vartheta)}+\frac{N^{-}(\vartheta, \varphi)}{N_{i n}^{-}(\vartheta)}\right]=1+a_{0} \sin \varphi+b_{0} \cos \varphi+c_{0} \sin 2 \varphi+d_{0} \cos 2 \varphi
$$

Because the false asymmetries are the same for plus and minus beam helicity states, they directly cancel in the calculation of the polarisation transfer components, and are therefore not important in the present measurement. However, if the dual analyser configuration of the FPP is used in future experiments to extract the helicity-independent induced polarisation components, a knowledge of these terms will be useful. Table 6.1 shows the four lowest Fourier amplitudes determined from the calibration data using eq. 6.15 compared with those obtained from recent e99007 single analyser data [71].

\subsection{Spin Transport}

The recoil proton polarisation observables at the focal plane must be related to the polarisations which give rise to them in the primary reaction at the target. To do so, one must be able to account for the rotation of the proton spin vector from the focal plane to a particular reference frame at the target. This rotation can be divided into two parts, both of which must be calculated on an event-by-event basis: the precession of the proton's spin in the magnetic elements of the HRS, and a transformation to the appropriate reference frame which depends on the reaction of interest. 


\subsubsection{Simple Dipole Approximation}

When considering the spin precession in the spectrometer optics, it is important to note that different proton trajectories through the HRS have associated with them different magnetic fields, and a dedicated model of the spectrometer optics is required. However, by far the most significant precession takes place in the dipole, and it is therefore a good approximation to work with the assumption that the HRS optics consist only of a perfect dipole field (one which is purely homogeneous with no fringe components). This also serves as a good introduction to the concept of spin transport in a magnetic system before moving on to a more rigorous treatment.

If the proton spin vector at the focal plane (with the axes defined in fig. 4.7) for a particular event is $\mathbf{P}^{\mathbf{f p p}}$, its equivalent at the target is given by:

$$
\mathbf{P}=\mathbf{S} \mathbf{P}^{\mathrm{fpp}}
$$

where $\mathbf{S}$ is the Spin Transport Matrix (STM). This matrix depends on the angle through which the proton spin vector precesses, which is formally defined as the difference between the momentum and spin rotations and is given by:

$$
\chi=\Theta_{\text {bend }} \frac{E_{P}}{M}\left(\frac{\mu_{p}}{\mu_{B}}-1\right),
$$

where $E_{P}, M$ and $\mu_{p}=2.79 \mu_{B}$ are the energy, mass and magnetic moment of the proton, respectively ( $\mu_{B}$ is the Bohr magneton). The nominal total bend angle in the HRS dispersive plane is $\Theta_{\text {bend }}=45^{\circ}$.

The STM is then given in terms of the precession angle by:

$$
\mathbf{S}=\left(\begin{array}{ccc}
\cos \chi & 0 & -\sin \chi \\
0 & 1 & 0 \\
\sin \chi & 0 & \cos \chi
\end{array}\right)
$$

By considering this simple dipole matrix, one is able to obtain the salient features of the spin precession associated with the present measurement, where the proton kinetic energy was chosen to be $E_{p}=3.0 \mathrm{GeV}$, for which the equivalent precession angle is approximately $270^{\circ}$ :

1. There is no precession of the $y$-component of the spin in the non-dispersive plane. 

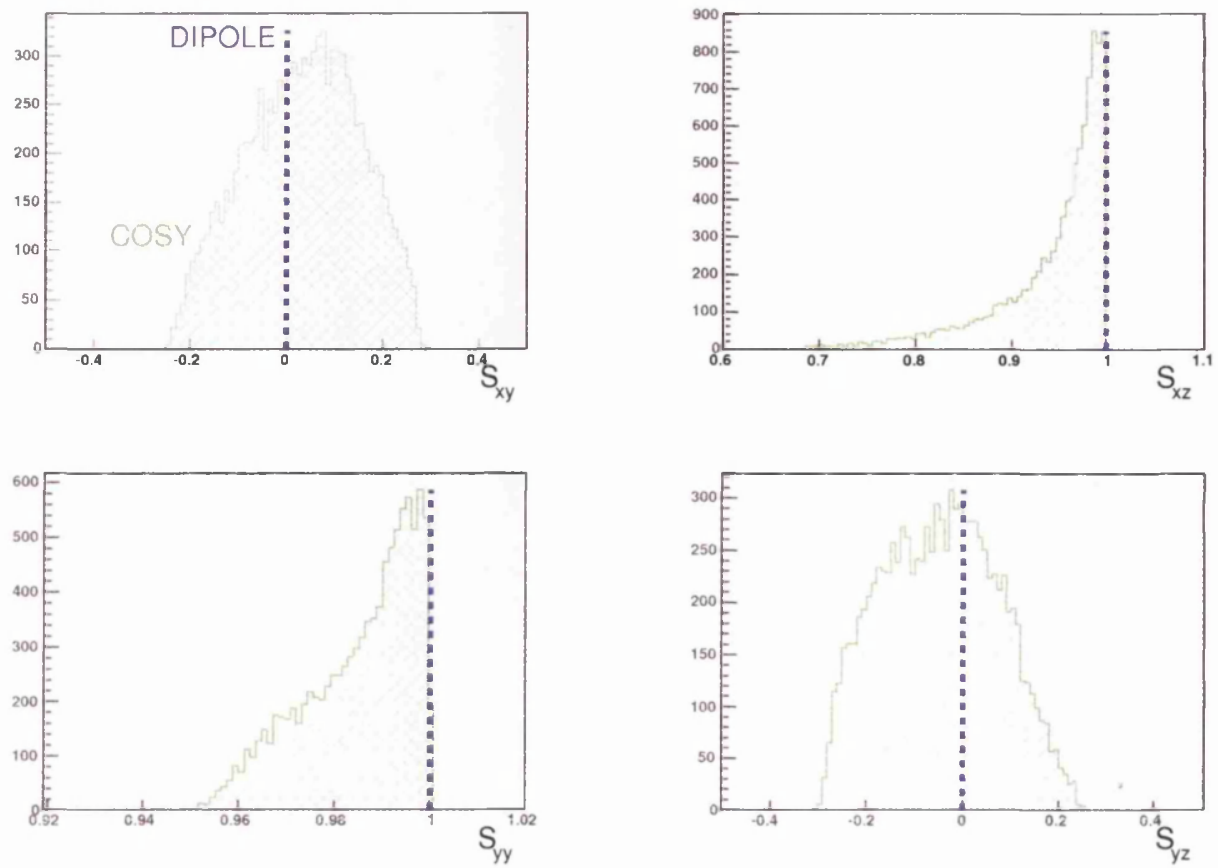

Figure 6.7: The comparison between the STM elements $\left(S_{x y}, S_{x z}, S_{y y}\right.$ and $\left.S_{y z}\right)$ calculated from the simple dipole model (blue) and full COSY calculation (green) for the current value of proton momentum.

2. The $x$ and $z$ components are precessed through the angle $\chi$ in the dispersive plane, so that:

$$
\begin{aligned}
& P_{x}=P_{z}^{f p p} \\
& P_{z}=-P_{x}^{f p p}
\end{aligned}
$$

The $z$-component of the spin vector at the target in this coordinate system is related to the longitudinal observable of primary interest. Therefore, the particular value of proton energy and precession angle were chosen for the current measurement to optimise the precession for measurement of this component ${ }^{3}$.

\subsubsection{COSY Model}

The physical situation is clearly much more complicated than the simplistic model described above since the effects of the HRS quadrupoles and the dipole

\footnotetext{
${ }^{3}$ At the expense of the $x$ or normal component which is of no real interest.
} 
fringe fields must also be considered. One of the reasons the previous model is so simple to consider is because all the spin rotations are in the trajectory plane, so the total precession angle can be calculated from the difference between initial and final trajectories alone. However, a rigorous calculation of the STM for the full spectrometer optics requires the knowledge of all intermediate rotations. A model of the spectrometer has been developed for this purpose using the differential-algebra based software package COSY [86], and based upon numerous HRS measurements and optical studies.

COSY is a hybrid magnetic modelling code which uses Taylor expansions to describe the action of a magnetic system and numerical integrators to determine actual trajectories. As a result, it is both faster than traditional ray-tracing codes and has the potential for much greater accuracy than most simple Taylor map applications. The spectrometer model which is input into COSY includes physical dimensions and field characteristics of the three quadrupoles and the dipole magnet, including the non-uniform fringe fields at the entrance and exit faces of the dipole, which were measured during the Hall commissioning. Deviations from the simple dipole model arise as a result of the introduction of non-zero field components in the transverse plane. These are due to the dipole fringe fields and higher order effects in the quadrupoles, where the effects are most pronounced at high excitation current and for extreme trajectories.

The COSY model is used to calculate the STM in the same transport coordinate system that is used in the target coordinate reconstruction described in sec. 5.2.2. As before, the proton trajectory through the HRS is parameterised in terms of the four proton trajectory variables $(y, \theta, \phi$ and $\delta)$. The rotation matrix that is calculated by COSY is parameterised in terms of expansion coefficients $\left(C_{i j}^{k l m n p}\right)$ in these target variables, which are determined to fifth order. The individual STM elements are then simply given by:

$$
S_{i j}=\sum_{k, l, m, n, p} C_{i j}^{k l m n p} x^{k} \theta^{l} y^{m} \phi^{n} \delta^{p}
$$

The variation in the STM elements of interest due to the variation in proton trajectory through the HRS as determined from the COSY model (relative to the equivalent simple dipole elements) can be seen in fig. 6.7. The deviations from the simple dipole treatment for all of the elements are quite pronounced, which has a significant effect on the final result. A large amount of work has been completed during several recent Hall A polarisation experiments to improve the 
COSY model, as well as minimise the systematic errors associated with obtaining the STM. The first such study involved minimising the actual error on the matrix by studying the relative alignment of the quadrupoles [87], while the second was related to the actual model dependency and the source of the remaining uncertainties [88].

\subsubsection{Coordinate Systems at the Target}

For physical interpretation and comparison with theoretical predictions, one must then transform the spin vector into the desired reference frame. Because the proton is not massless it's polarisation is frame dependent, and it is conventional to quote it in the rest frame of the proton. Therefore, the final results are rest frame polarisation components relative to the axes as defined in a particular reference frame. As a result, while the calculation of the spin precession in the HRS is the more complicated of the two rotations which contribute to the full STM, the rotation to the reference frame of interest is a little more subtle than one might initially expect.

\section{Scattering Frame}

For the elastic ep scattering data which is used for calibration of the FPP analysing power (sec. 6.4) the polarisation is expressed in the electron-proton laboratory scattering frame. In terms of the laboratory kinematic variables, the axes are defined by the unit vectors:

$$
\begin{aligned}
& \hat{x}=\hat{z} \times \hat{y}, \\
& \hat{y}=\frac{\mathbf{q} \times \mathbf{k}_{\mathbf{i}}}{\left|\mathbf{q} \times \mathbf{k}_{\mathbf{i}}\right|}, \\
& \hat{z}=\frac{\mathbf{q}}{|\mathbf{q}|},
\end{aligned}
$$

which is illustrated in fig. 6.8. Here, $\mathbf{k}_{\mathbf{i}}$ is the incident electron momentum and $\mathbf{q}$ 'is the momentum transferred to the proton.

\section{Transformation to the Centre-of-Mass Frame}

Similarly, the analysis of the production data for Compton scattering events involves a rotation to the $\gamma p$ laboratory scattering frame, which is then followed by 


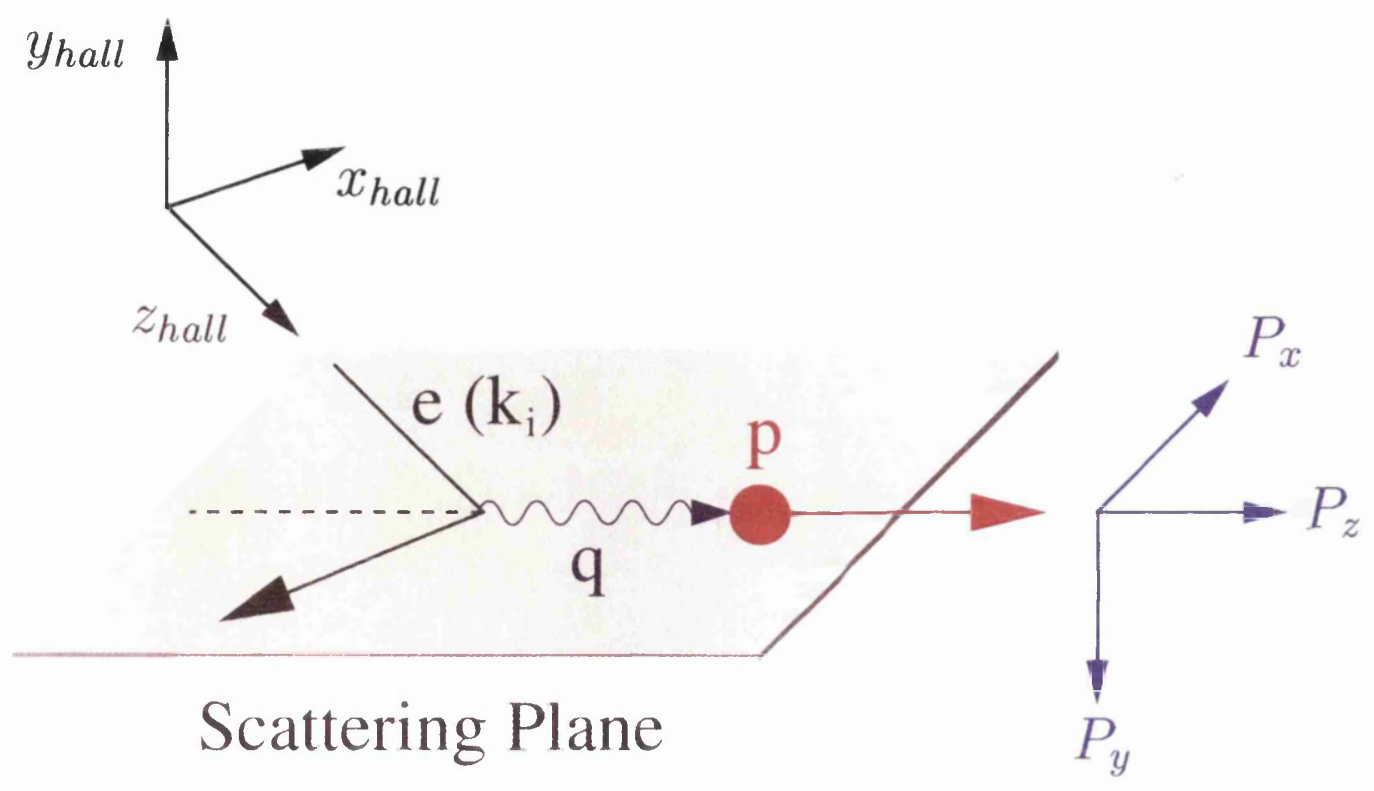

Figure 6.8: Definition of the proton polarisation components at the target (eq.6.20) in the elastic ep scattering frame.

a Lorentz transformation from the laboratory to the CM frame defined in eq. 3.7 and fig. 3.3. This is complicated slightly by the fact that, by convention, the axes in the $\gamma p$ scattering frame are not the same as in eq. 6.20 above, but are given by:

$$
\hat{x}_{\gamma p}=-\hat{x}_{e p}, \quad \hat{y}_{\gamma p}=-\hat{y}_{e p}, \quad \hat{z}_{\gamma p}=\hat{z}_{e p} .
$$

The transformation from this laboratory frame to the CM turns out to be a rather simple exercise in relativistic kinematics. For readers who are unfarniliar with the treatment of polarisation phenomena in a relativistic framework, a good introduction can be found in ref. [89], whereas the specific details of this particular transformation can be found in ref. $[35,90]$.

The proton spin can be expressed in terms of the four-vector:

$$
P_{\mu}=\left(P_{0}, P_{x}, P_{y}, P_{z}\right)
$$

where the $P_{0}$ component represents the Lorentz invariant magnitude of the vector. The transformation of this vector from the laboratory to CM frame involves a series of boosts and rotations, and can be broken down into five steps:

1. A boost along the proton momentum from the proton rest frame to the 
laboratory system,

$$
\left(\begin{array}{cccc}
\gamma_{l a b} & 0 & 0 & \beta_{l a b} \gamma_{l a b} \\
0 & 1 & 0 & 0 \\
0 & 0 & 1 & 0 \\
\beta_{l a b} \gamma_{l a b} & 0 & 0 & \gamma_{l a b}
\end{array}\right)
$$

2. A rotation which brings the $z$-axis parallel to the incident fhoton momentum in the laboratory, where $\theta_{p}$ is the proton scattering argle (nominally equal to $\Theta_{H R S}$ in table 5.2 and fig. A.1),

$$
\left(\begin{array}{cccc}
1 & 0 & 0 & 0 \\
0 & \cos \theta_{p} & 0 & \sin \theta_{p} \\
0 & 0 & 1 & 0 \\
0 & -\sin \theta_{p} & 0 & \cos \theta_{p}
\end{array}\right)
$$

3. A boost along the initial photon direction to the CM frame, with $\beta_{c m}$ representing the velocity of the CM frame as seen from the laboratory,

$$
\left(\begin{array}{cccc}
\gamma_{c m} & 0 & 0 & -\beta_{c m} \gamma_{c m} \\
0 & 1 & 0 & 0 \\
0 & 0 & 1 & 0 \\
-\beta_{c m} \gamma_{c m} & 0 & 0 & \gamma_{c m}
\end{array}\right)
$$

4. A rotation which brings the $z$-axis parallel to the recoil proton momentum in the CM frame,

$$
\left(\begin{array}{cccc}
1 & 0 & 0 & 0 \\
0 & \cos \theta_{c m} & 0 & \sin \theta_{c m} \\
0 & 0 & 1 & 0 \\
0 & -\sin \theta_{c m} & 0 & \cos \theta_{c m}
\end{array}\right)
$$

5. A boost along the recoil proton momentum to proton rest frame in the $\mathrm{CM}$ 
coordinate system,

$$
\left(\begin{array}{cccc}
\gamma_{c m} & 0 & 0 & -\beta_{c m} \gamma_{c m} \\
0 & 1 & 0 & 0 \\
0 & 0 & 1 & 0 \\
-\beta_{c m} \gamma_{c m} & 0 & 0 & \gamma_{c m}
\end{array}\right)
$$

It has long been known that three separate Lorentz boosts on a particle-at-rest system results in a purely in-plane rotation. This means that the net effect of the individual transformations given by eq. $6.23-6.27$ is that $P_{0}$ (as expected) and $P_{y}$ remain unchanged, and there is a mixing of the in-plane components described by:

$$
\left(\begin{array}{c}
P_{0} \\
P_{x} \\
P_{y} \\
P_{z}
\end{array}\right)_{C M}=\left(\begin{array}{cccc}
1 & 0 & 0 & 0 \\
0 & \cos \theta_{r o t} & 0 & \sin \theta_{r o t} \\
0 & 0 & 1 & 0 \\
0 & -\sin \theta_{r o t} & 0 & \cos \theta_{r o t}
\end{array}\right)\left(\begin{array}{c}
P_{0} \\
P_{x} \\
P_{y} \\
P_{z}
\end{array}\right)_{l a b},
$$

where $\theta_{\text {rot }}$ must be calculated on an event-by-event basis, and depends only on the recoil proton kinematic quantities. For the present data-set the average in-plane rotation between the $\gamma p$ laboratory and CM systems was found to be $\theta_{\text {rot }}=20.2^{\circ}$.

\subsection{Maximum likelihood Determination of Polarisation Observables}

The measured azimuthal asymmetries together with the spin transport matrix obtained from the COSY model can be used to determine the proton polarisation observables in the appropriate reference frame at the target. It is not instantly obvious that a measurement of two polarisation components at the focal plane can lead to the determination of all three components at the target. In fact, it is possible to extract three induced $(\mathbf{P})$ and three transferred $\left(\mathbf{P}^{\prime}\right)$ polarisation components separately by utilising not only the helicity-difference, but also the helicity-sum azimuthal distributions. Clearly no exact solution to such a problem exists, but a method of parameter estimates based upon the maximum likelihood technique has been developed for this purpose.

We begin by defining a likelihood function as a measure of the probability for obtaining the asymmetry given by eq. 6.12. The function is constructed by 
taking the product over $N$ total events of all the individual probabilities:

$$
L=\prod_{i=1}^{N} f\left(\varphi_{i}, \vartheta_{i} ; \mathbf{P}, \mathbf{P}^{\prime}\right)
$$

Within a sample of protons with focal plane polarisations $P_{x}^{f p p}$ and $P_{y}^{f p p}$, which are measured in a polarimeter with analysing power $A_{y}$ and first-order instrumental asymmetries $a_{0}$ and $b_{0}$, the probability that the $i$ th proton scatters through the angles $\left(\varphi_{i}, \vartheta_{i}\right)$ is

$$
f\left(\varphi_{i}, \vartheta_{i}\right)=\frac{1}{2 \pi}\left[1+a\left(\vartheta_{i}\right) \cos \varphi_{i}+b\left(\vartheta_{i}\right) \sin \varphi_{i}\right]
$$

where

$$
\begin{aligned}
& a\left(\vartheta_{i}\right)=a_{0}-A_{y}\left(\vartheta_{i}\right) P_{x}^{f p p} \\
& b\left(\vartheta_{i}\right)=b_{0}+A_{y}\left(\vartheta_{i}\right) P_{y}^{f p p}
\end{aligned}
$$

The azimuthal asymmetries depend most crucially on the focal plane polarisations, which can be represented by linear functions of the transferred and induced polarisations at the target, and the analysing power. However, the variation of the spin transport matrix (S) on an event-by-event basis, due to the variation in the proton trajectories through the HRS, must also be taken into account. This is achieved by assigning a weight to each event proportional to the appropriate STM element $\left(S_{i j}\right)$ so that eq. 6.31 becomes:

$$
\begin{array}{ccc}
a(\vartheta)=a_{0}-A_{y}(\vartheta) \quad[ & S_{x x}\left(P_{x}+h P_{x}^{\prime}\right)+S_{x y}\left(P_{y}+h P_{y}^{\prime}\right) \\
& \left.+S_{x z}\left(P_{z}+h P_{z}^{\prime}\right)\right] \\
b(\vartheta)=b_{0}+A_{y}(\vartheta) \quad\left[\begin{array}{l}
S_{y x}\left(P_{x}+h P_{x}^{\prime}\right)+S_{y y}\left(P_{y}+h P_{y}^{\prime}\right) \\
+
\end{array}\right. \\
\left.+S_{y z}\left(P_{z}+h P_{z}^{\prime}\right)\right],
\end{array}
$$

where $P_{k}$ and $P_{k}^{\prime}(k=x, y, z)$ are the induced and transferred polarisation components, respectively, and $h$ is the value of beam polarisation ${ }^{4}$.

It is now possible to rewrite the likelihood function in eq. 6.29 by separating

\footnotetext{
${ }^{4}$ To obtain the physical induced polarisations one does not need to divide by $h$ since they are helicity-independent.
} 
the transferred and induced polarisation, and the instrumental asymmetries:

$$
L=\prod_{i=1}^{N} \frac{1}{2 \pi}\left(1+\lambda_{0, i}+\sum_{k} \lambda_{k, i} \hat{P}_{k}+\sum_{k} \lambda_{k, i}^{\prime} \hat{P}_{k}^{\prime}\right)
$$

where

$$
\begin{array}{ll}
\lambda_{x}=A_{y}(\vartheta)\left(S_{y x} \cos \varphi-S_{x x} \sin \varphi\right), & \lambda_{x}^{\prime}=-h \lambda_{x} \\
\lambda_{y}=A_{y}(\vartheta)\left(S_{y y} \cos \varphi-S_{x y} \sin \varphi\right), & \lambda_{y}^{\prime}=-h \dot{\lambda}_{y} \\
\lambda_{z}=A_{y}(\vartheta)\left(S_{y z} \cos \varphi-S_{x z} \sin \varphi\right), & \lambda_{z}^{\prime}=-h \dot{\lambda}_{z}
\end{array}
$$

and

$$
\lambda_{0}=1+a_{0} \sin \varphi+b_{0} \cos \varphi+c_{0} \sin 2 \varphi+d_{0} \cos 2 \varphi .
$$

The likelihood function in eq. 6.33 reaches a maximum value when the polarisation estimates $\left(\hat{P}_{k}\right.$ and $\left.\hat{P}_{k}^{\prime}\right)$ satisfy the solution to the system of coupled nonlinear equations:

$$
\begin{aligned}
& \frac{\partial \ln L}{\partial \hat{P}_{k}}=0, \\
& \frac{\partial \ln L}{\partial \hat{P}_{k}^{\prime}}=0 .
\end{aligned}
$$

Obtaining the solutions for such a system of equations is far from trivial, but the problem can be simplified using a linearisation technique [91]:

$$
\underbrace{\left(\begin{array}{c}
\sum \lambda_{x}\left(1-\lambda_{0}\right) \\
\sum \lambda_{y}\left(1-\lambda_{0}\right) \\
\vdots \\
\sum \lambda_{z}^{\prime}\left(1-\lambda_{0}\right)
\end{array}\right)}_{\mathbf{B}}=\underbrace{\left(\begin{array}{cccc}
\sum \lambda_{x} \lambda_{x} & \sum \lambda_{x} \lambda_{y} & \cdots & \sum \lambda_{x} \lambda_{z}^{\prime} \\
\sum \lambda_{y} \lambda_{x} & \sum \lambda_{y} \lambda_{y} & \cdots & \sum \lambda_{y} \lambda_{z}^{\prime} \\
\vdots & \vdots & \ddots & \vdots \\
\sum \lambda_{z}^{\prime} \lambda_{x} & \sum \lambda_{z}^{\prime} \lambda_{x} & \cdots & \sum \lambda_{z}^{\prime} \lambda_{z}^{\prime}
\end{array}\right)}_{\mathbf{F}}\left(\begin{array}{c}
\hat{P}_{x} \\
\hat{P}_{y} \\
\vdots \\
\hat{P}_{z}^{\prime}
\end{array}\right)
$$

The estimates for the six proton polarisation observables at the target are then given by:

$$
\left(\hat{P}_{x}, \hat{P}_{y}, \cdots, \hat{P}_{z}^{\prime}\right)^{T}=\mathbf{F}^{-\mathbf{1}} \mathbf{B}
$$

For the purpose of the present measurement of Compton scattering observables, only the polarisations $P_{x}^{\prime}=K_{L T}$ and $P_{z}^{\prime}=K_{L L}$ in the CM frame are of interest. 
Their statistical errors are obtained from the covariance matrix $\mathbf{F}^{-\mathbf{1}}$.

\subsection{Analysing Power Calibration}

Because the FPP has not previously been used in the dual analyser configuration, a significant portion of the beamtime allocated for the polarised RCS measurement was set aside to accumulate $p\left(\vec{e}, e^{\prime} \vec{p}\right)$ calibration data (the analysis of which was described in sec. 5.5). With the techniques for measuring the azimuthal asymmetries and modelling the HRS spin transport already discussed, only one other step is required to extract the polarisation transfer in Compton scattering: measurement of the $\mathrm{CH}_{2}$ and carbon analysing powers. The analysing power combined with the polarimeter efficiency gives the figure-of-merit (FOM), which directly relates the statistical uncertainty one obtains in a polarisation measurement to the total number of protons in the data-set. The FOM is therefore a very useful quantity for comparison between the new and old FPP configurations, as well as for planning new high proton momentum experiments. In addition, as we saw in sec. 6.1.4, the elastic ep data also allows determination of the polarimeter instrumental asymmetries.

So what is it that makes the elastic $e p$ reaction so well suited to calibration of the FPP analysing power? The Sachs form factor ratio $\left(G_{E} / G_{M}\right)$ for the proton can be calculated from a simultaneous measurement of the transverse and longitudinal $e p$ polarisation transfer components, without the need for knowledge of the beam polarisation or analysing power. Therefore, by selecting the elastic $e p$ events identified in sec. 5.5, the maximum likelihood method can be used to simultaneously extract the quantities $h A_{y} P_{x}^{\prime}$ and $h A_{y} P_{z}^{\prime}$ in the scattering plane (where $h$ and $A_{Y}$ are set to unity since they cancel out in the calculation anyway). These are related to the Sachs form factor ratio, using the same notation as in sec. 2.1 , by:

$$
\frac{G_{E}}{G_{M}}=-\frac{h A_{y} P_{x}^{\prime}}{h A_{y} P_{z}^{\prime}} \frac{E+E^{\prime}}{2 M} \tan \frac{\theta}{2} .
$$

The value obtained for this ratio in the present measurement is shown in fig. 6.9 relative to results from two previous Hall A experiments (e93027 [92] and e99007 [70]) which have both utilised the same recoil polarimetry method, although with different experimental arrangements and different FPP configurations. There is good agreement with the trend identified by these previous results, although the statistical accuracy is clearly not as good. 


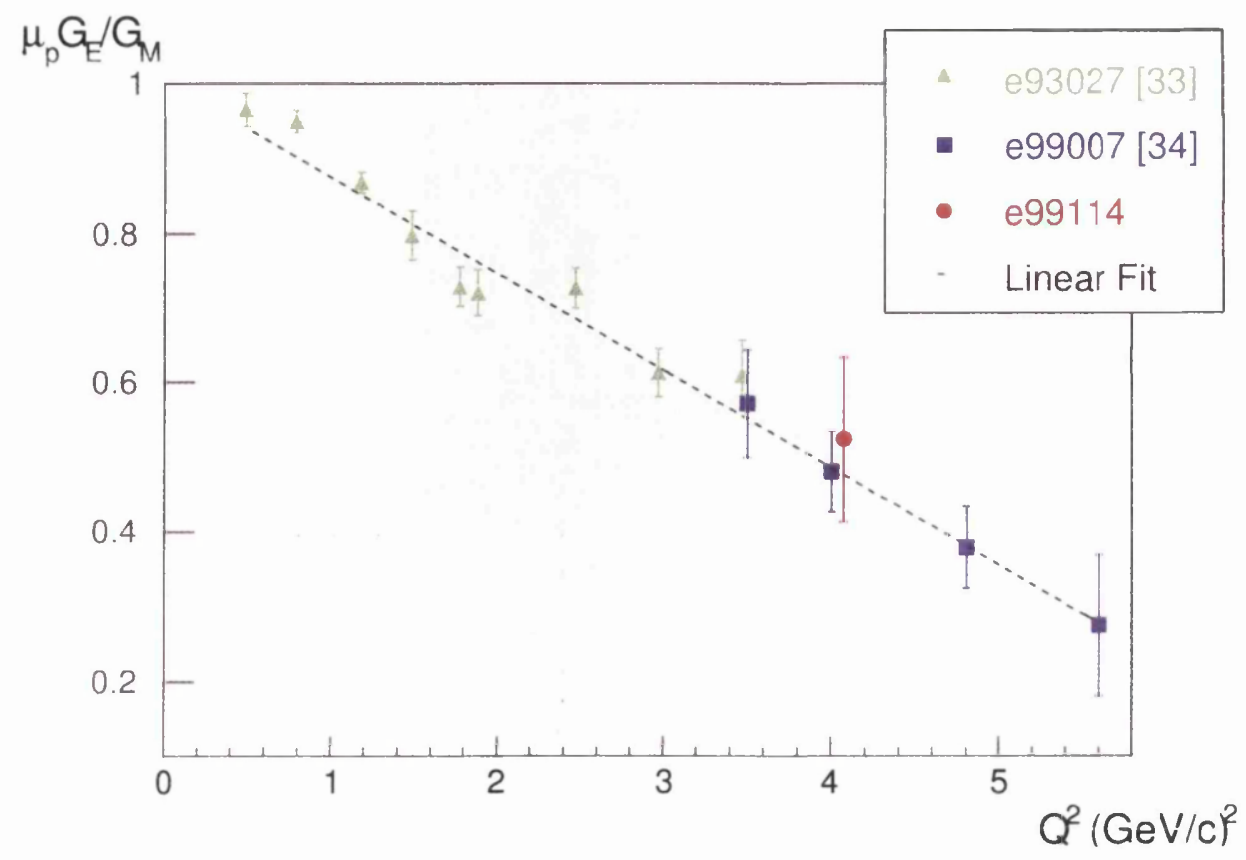

Figure 6.9: The experimentally determined Sachs form factor ratio for the proton $\mu_{p} G_{E} / G_{M}$ as a function of four-momentum transfer $Q^{2}$ for the e99114 (red), e99007 (blue) and e93027 (green) data (only statistical errors are shown). A straight-line fit obtained from the previous two data-sets is also shown.

The over-determined nature of this reaction means that the form factor ratio, once extracted from the data, can be used to obtain the experimental analysing power as a function of $\vartheta$. It has been shown, in ref. [71] for example, that the polarisation transfer components can be re-written in terms of the form factor ratio and kinematic factors alone:

$$
\begin{aligned}
P_{x}^{\prime} & =\frac{-2 \sqrt{\tau(1+\tau)} \tan \frac{\theta}{2} \frac{G_{E}}{G}}{\left(\frac{G_{E}}{G_{M}}\right)^{2}+(\tau / \epsilon)}, \\
P_{z}^{\prime} & =\frac{\frac{E+E^{\prime}}{M} \sqrt{\tau(1+\tau)} \tan ^{2} \frac{\theta}{2}}{\left(\frac{G_{E}}{G_{M}}\right)^{2}+(\tau / \epsilon)},
\end{aligned}
$$

where the term $1 / \epsilon=1+2(1+\tau) \tan (\theta / 2)$ represents the polarisation of the virtual photon exchanged in the primary reaction. It should now be clear that, as long as the electron beam polarisation is known (Appendix B), the experimentally determined analysing power $\left(A_{y}\right)$ may be obtained from a comparison between the measured value of $h A_{y} P_{z}^{\prime}$ and the value calculated using eq. 6.40. 
Using this technique, the analysing power of both analysers have been calculated in discrete bins of polar scattering angle $(\vartheta)$, the bin widths being chosen to ensure that the statistical error in $A_{y}$ for each bin was approximately equal. The results for $A_{y}(\vartheta)$ for both the $\mathrm{CH}_{2}$ and carbon analysers are shown in fig. 6.10. with previous results for similar proton kinetic energies included for comparison [93]. One should also note that the dashed line on these graphs more clearly shows the variation of the analysing power with $\vartheta$ than the calibration data olone. These parameterisations have been obtained from a fit to the $\pi^{0}$ background polarisation production data, for which the statistical accuracy is extremely good.

The $\vartheta$-dependence of the analysing power exhibits similar behavior for both $\mathrm{CH}_{2}$ and carbon analysers. As expected, the effective analysing power is close to zero at small scattering angles where Coulomb scattering dominates, then rises to a maximum value before falling off towards zero. Furthermore, it is clear that the presence of the hydrogen in the $\mathrm{CH}_{2}$ analyser has the effect of increasing magnitude of the analysing power relative to the carbon at small angles, while causing a sharper fall at larger angles. Data from the two previous Hall A form factor experiments (e93027 and e99007) have also been included in fig. 6.10 for comparison. The former experiment used the standard single carbon arrangement of the FPP, while the latter used a slightly modified configuration with $100 \mathrm{~cm}$ of $\mathrm{CH}_{2}$ (as described in sec. 4.3.2). The conclusion of the Dubna tests [72], that the magnitude of the $\mathrm{CH}_{2}$ analysing power is largely unchanged by an decrease in thickness, is supported by comparison between the $44 \mathrm{~cm}$ e99114 data and the $100 \mathrm{~cm}$ e99007 data for similar values of proton momentum. Comparison with previous carbon data, on the other hand, which was taken with lower momentum protons suggests that the analysing power becomes systematically lower, and decreases more rapidly, as the proton momentum gets larger.

One of the unknown aspects regarding the new double analyser FPP is the effect scattering via the strong interaction in the $\mathrm{CH}_{2}$ has on the proton polarisations at the focal plane. Or, put another way, it is not known whether there will be any significant depolarisation of the recoil protons as they pass through the $\mathrm{CH}_{2}$ prior to scattering in the carbon analyser. One way to examine how large this effect might be is to measure by how much the carbon analysing power changes when only small angle scattering events in the $\mathrm{CH}_{2}$ (i.e. Coulomb events) are selected. The observation that the resultant change in analysing power was very small, around $1 \%$ in fact, implies that depolarisation of the proton sample in the $\mathrm{CH}_{2}$ is not a problem. 

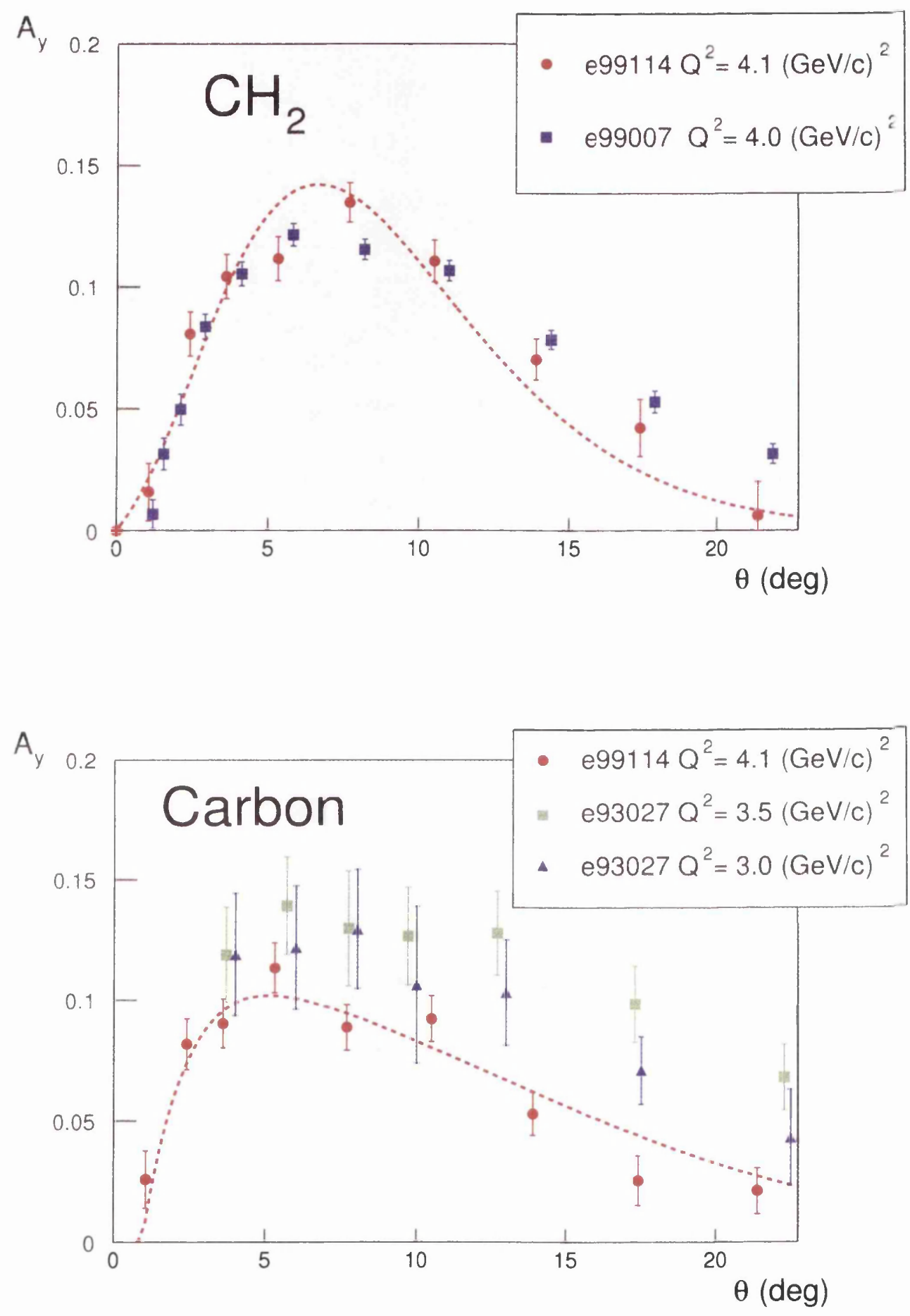

Figure 6.10: Experimentally determined analysing power $\left(A_{y}(\vartheta)\right.$ ) for $\mathrm{CH}_{2}$ (c.f. e99007) and carbon (c.f. e93027) data. On both plots the present data are shown in red and small $\vartheta$ offsets have been applied for clarity. 


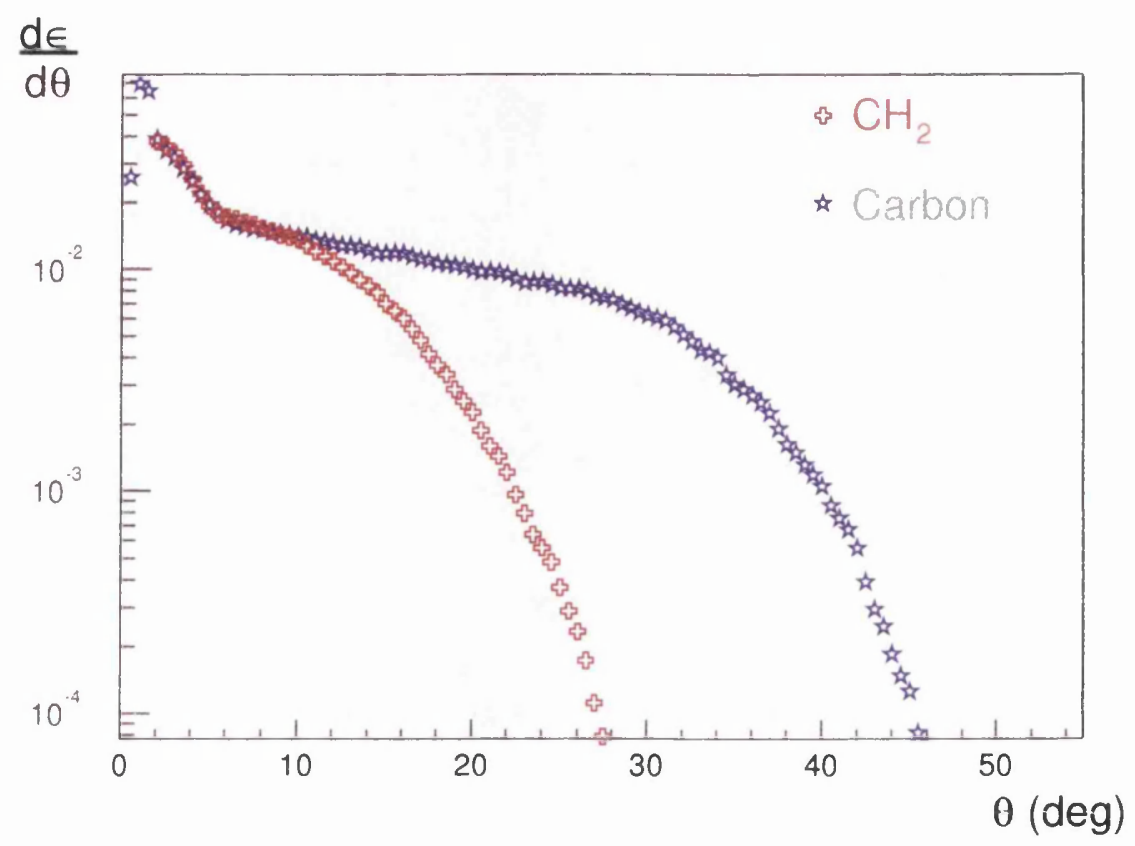

Figure 6.11: Measured differential efficiency $\frac{d \varepsilon}{d \vartheta}$ for the $\mathrm{CH}_{2}$ (red) and carbon (blue) polarimeters. The drop in $\mathrm{CH}_{2}$ efficiency at larger scattering angles is due to the limited size of the front FPP chambers.

\section{Figure of Merit}

The precision which may be obtained in a polarisation measurement depends on the analysing power, the acceptance and the detection efficiency of the polarimeter. Thus, when assessing the effectiveness of such a device it is convenient to define a Figure-of-Merit (FOM). This has previously been determined for the single analyser configuration of the FPP at similar proton momenta, and therefore acts as a good measure of the improvement after the rearrangement into dual analyser mode.

The FPP efficiency for a given scattering angle $(\vartheta)$ is given by the ratio of the number of successfully reconstructed outgoing tracks (passing the various cuts) to the number of incoming tracks:

$$
\varepsilon(\vartheta)=\frac{N_{\text {out }}(\vartheta)}{N_{\text {in }}}
$$

The differential efficiency $(d \varepsilon / d \vartheta)$ has been measured using the calibration data for both $\mathrm{CH}_{2}$ and carbon polarimeters, and is shown in fig. 6.11. The most significant feature regarding this plot is the disparity between the carbon and 


\begin{tabular}{|c|c|c|}
\hline & $\mathrm{CH}_{2}$ & Carbon \\
\hline \hline$\vartheta$ Range & $0.8^{\circ} \leq \vartheta \leq 24^{\circ}$ & $0.8^{\circ} \leq \vartheta \leq 70^{\circ}$ \\
\hline$A_{y}$ & $0.0836 \pm 0.0031$ & $0.0570 \pm 0.0029$ \\
\hline FOM & $2.94 \times 10^{-3}$ & $1.61 \times 10^{-3}$ \\
\hline
\end{tabular}

Table 6.2: The polar scattering angle range, the average analysing power and FOM for both polarimeters.

$\mathrm{CH}_{2}$ efficiency at larger scattering angles, where the smaller size of the front FPP chambers severely limits the $\mathrm{CH}_{2}$ efficiency.

The efficiency alone is not sufficient to characterise the statistical effectiveness of the polarimeter, since not all of the outgoing tracks will contribute to the focal plane asymmetry. Indeed, only the fraction of those events which undergo strong nuclear scattering in the analyser will contribute significantly. Therefore, the analysing power, or more accurately the square of the analysing power, must also be taken into account. The FOM and the number of good events which will contribute to the asymmetry are then related to the number of incoming events by the equation:

$$
N=N_{i n} \int_{\vartheta_{\min }}^{\vartheta_{\max }} \epsilon(\vartheta) A_{y}^{2}(\vartheta) d \vartheta=N_{0} . F O M
$$

The observation that the carbon analysing power is not affected by scattering in the $\mathrm{CH}_{2}$ means that we can analyse the data from the two polarimeters as though they were two completely independent measurements of the same physical quantity. One can then compare the average values for the analysing power and FOM of both polarimeters over a given polar scattering angle range, and shown in table 6.2, with the values measured in previous experiments. The combined FOM in the new dual-analyser configuration was found to be $4.5 \times 10^{-3}$, which is a considerable improvement over the value of $2.9 \times 10^{-3}$ that was obtained using the e99007 single analyser FPP configuration at similar proton momenta. Such an improvement results in a substantial reduction in the statistical uncertainty associated with the measurement. 


\section{Chapter 7}

\section{Results and Discussion}

In the last chapters we have discussed the calibration of the various detector components, the methods of identifying the RCS events and the methods employed to extract the recoil proton polarisation observables. We now turn our attention to the presentation of the results of interest, namely the Compton spin observibles in the CM frame. This discussion includes a description of the background correction required to extract these results, and a thorough analysis of the experimental uncertainties. Finally, we present a discussion of the results within the orerall context of the measurement, and the conclusions that can be made regardiny the Compton scattering reaction mechanism and structure of the proton. For completeness, the polarisation transfer results obtained from independent analyis of the $\mathrm{CH}_{2}$ and carbon data are provided in this chapter. In all cases, the results from the two polarimeters agree to within the overall statistical accuracy.

\subsection{Raw Polarisation Results}

We have already seen that the RCS events of interest are located in a small elliptical region around $\delta x=\delta y=0$ (in fig. 5.5). Calculating the polarisation transfer observables for events in this region, therefore, allows access to $K_{L I}$ and $K_{L T}$ (as defined in eq. 3.7) through the background corrections:

$$
\begin{aligned}
K_{L T} & =\frac{1}{D}\left[K_{L T}^{t o t}-(1-D) K_{L T}^{b a c k}\right] \\
K_{L L} & =\frac{1}{D}\left[K_{L L}^{t o t}-(1-D) K_{L L}^{b a c k}\right]
\end{aligned}
$$


where the tot and back superscripts label the polarisation corresponding to the total events in the chosen elliptical region and the $\pi^{0}$ background events, respectively. The significant presence of background events under the Compton peak causes the RCS polarisation to be diluted. To take this into account, the dilution factor $(D)$ is included in eq. 7.1, and is simply the ratio of RCS to total events in this region ${ }^{1}$.

\subsubsection{Background Polarisation}

The fact that the present experimental technique does not allow for further separation of the Compton and background events makes the correction of eq. 7.1 necessary. Obviously, the first quantities required are the polarisation transfer observables for events in the background distribution $\left(K_{L T}^{\text {back }}\right.$ and $\left.K_{L L}^{\text {back }}\right)$. For the present purposes all events in fig. 5.5, with the exception of those which fall within a $4 \sigma$ region around the RCS and ep peaks, are used in the calculation of the background polarisation. The techniques for using the FPP data for these purposes have been described in the previous chapter. In addition, the necessary correction for the incident photon beam polarisation is given in Appendix $B$. The following results have been obtained:

\begin{tabular}{|c|c|c|}
\hline & $\mathrm{CH}_{2}$ & Carbon \\
\hline$K_{L T}^{\text {back }}$ & $0.489 \pm 0.008$ & $0.463 \pm 0.011$ \\
\hline$K_{L L}^{\text {back }}$ & $0.528 \pm 0.008$ & $0.539 \pm 0.011$ \\
\hline
\end{tabular}

Simply calculating these values, however, is not enough. To interpolate underneath the Compton peak we have to assume the background polarisation is homogeneous. Even though the background is anticipated to be very uniform in nature, dominated as it is by $\pi^{0}$ events, the assumption of a constant background polarisation is one of the most critical factors in the calculation of the RCS observables. As such, it warrants a full examination of the stability of the background polarisation as a function of scattered photon energy $\left(E_{\text {calo }}\right)$, and over the $\delta x-\delta y$ phase space. The results of such an investigation are shown in fig. 7.1. The best estimate of the constancy of $K_{L T}^{b a c k}$ and $K_{L L}^{b a c k}$ is the value of $\chi^{2} / N D F$, calculated from the spread of the individual points from the average values given above, for each of the graphs in this figure. The results are mixed, but the the deviations from the average in some of the graphs, particularly both

\footnotetext{
${ }^{1}$ This dilution factor is calculated from the ratio $N_{\pi} / N_{r c s}$ by the means discussed in sec. 5.4.2.
} 

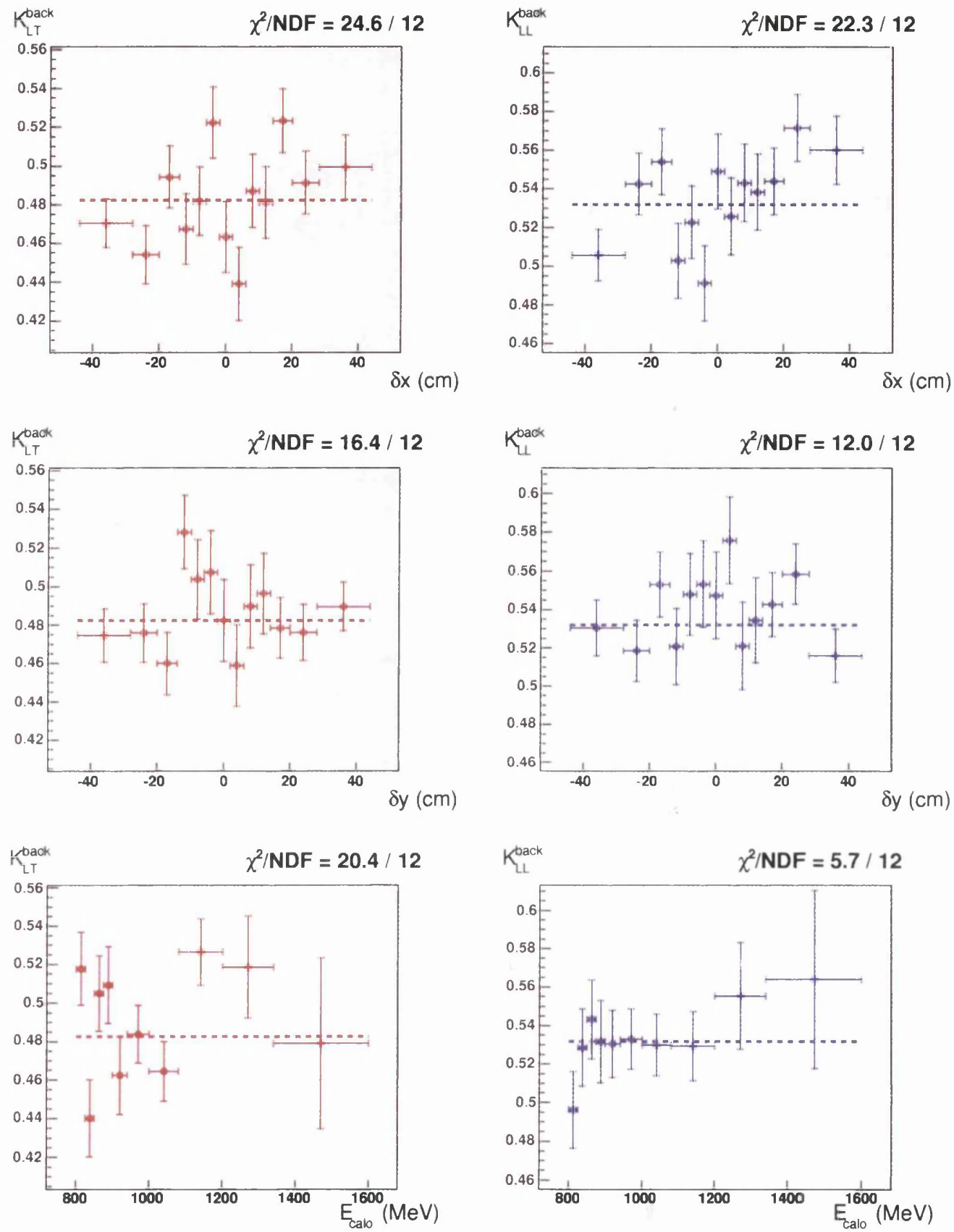

Figure 7.1: Homogeneity of the background polarisation observables $K_{L T}^{b a c k}$ and $K_{L L}^{\text {back }}$ over $\delta_{x}$ (top), $\delta_{y}$ (middle) and $E_{\text {calo }}$ (bottom). 
of the $\delta x$ ones, are larger than one might reasonably expect from a purely statistical perspective. This effect is likely, therefore, to contribute to the overall uncertainty of the measurement, and is discussed fully below in sec. 7.2.1.

\subsubsection{Final Results for $K_{L T}$ and $K_{L L}$}

The next step in obtaining the RCS results is the calculation of the polarisation transfer components for all events in the elliptical region at the centre of $\delta x-\delta y$. The first and most important question is related to the choice of the width of this elliptical region. It is clear from eq. 7.1 that the overall statistical accuracy of the measurement depends critically on this choice. There should therefore be some optimum width, which can be obtained by calculating the RCS polarisation transfer observables $\left(K_{L T}\right.$ and $\left.K_{L L}\right)$ for seven different choices of elliptical cutwidth (described in terms of $n_{\text {sigma }}$ : the number of $\sigma$ in both directions from the centre of the Compton peak) and observing the variation in the statistical error. These results are shown in fig. 7.2, from which three important observations can be made:

1. Naturally, the RCS contribution to the polarisation of all events in the chosen region is most pronounced when the cut is narrow. As the cut is opened up, this contribution becomes less significant and the total polarisation values $\left(K^{\text {tot }}\right)$ asymptotically approaches the background value $\left(K^{\text {back }}\right)$.

2. While the statistical error clearly varies as a function of $n_{s i g m a}$, the calculated values of $K_{L T}$ and $K_{L L}$ are equal, within the statistical accuracy, for each of the chosen values.

3. There is a distinct increase in the statistical error averaged over both polarisation components $(\bar{\sigma})$ either side of a minimum value around $2 n_{\text {sigma }}$. The exact value of this minimum is found by performing a fit on $\bar{\sigma}$ and finding the minimum of the fit function.

A value of $1.6 n_{\text {sigma }}$ was found to give the minimum statistical error, and the corresponding dilution factor for this cut-width was calculated:

$$
D=0.3825 \pm 0.0035
$$

The polarisation observables for all of the events in this region were: 



Figure 7.2: Variation of transverse (top) and longitudinal (middle) polarisation transfer components, as well as the corresponding average statistical error (bottom), for different $n_{\text {sigma }}$ cuts. The top two plots individually show the background (red line), total (red points) and RCS (blue points) polarisations. 


\begin{tabular}{|c|c|c|}
\hline & $\mathrm{CH}_{2}$ & Carbon \\
\hline$K_{L T}^{\text {tot }}$ & $0.357 \pm 0.036$ & $0.322 \pm 0.049$ \\
\hline$K_{L L}^{\text {tot }}$ & $0.587 \pm 0.036$ & $0.591 \pm 0.050$ \\
\hline
\end{tabular}

Finally, by taking a weighted average of the results from both polarimeters and applying the correction in eq. 7.1, the polarisation transfer components for Compton events were found:

$$
\begin{aligned}
& K_{L T}=0.112 \pm 0.078 \\
& K_{L L}=0.679 \pm 0.083
\end{aligned}
$$

\subsection{Systematic Uncertainties}

The fact that this measurement involves taking a beam-helicity asymmetry means that most of the possible systematic uncertainties directly cancel. That is not to say that there are no remaining significant systematic contributions to the overall uncertainty. Indeed, we have already seen that the assumption of a uniform background polarisation is open to some debate. What follows is a list of possible systematic effects and estimates of their respective contribution to the total experimental uncertainty.

\section{FPP Scattering Angles}

The accuracy with which the scattering angles used to form the beam-helicity asymmetries are measured in the FPP is known to be around $1 \mathrm{mrad}$. Therefore, to estimate the effect this uncertainty has on the final result, the data have been re-analysed with an offset of $+2 \mathrm{mrad}$ applied to the values of both polar $(\vartheta)$ and azimuthal $(\varphi)$ scattering angles. The resultant changes in the polarisation transfer components were found to be:

\begin{tabular}{|c|c|c|}
\hline & $\Delta K_{L T}$ & $\Delta K_{L L}$ \\
\hline$\vartheta(+2 \mathrm{mrad})$ & -0.002 & +0.008 \\
\hline$\phi(+2 \mathrm{mrad})$ & -0.001 & +0.000 \\
\hline
\end{tabular}

\section{Spin Transport}

The spin transport matrix elements are sensitive to uncertainties in the total bending angles determined by the COSY model used to describe the HRS. These 
have been examined in great detail by Pentchev et al. $[87,88]$ with regards to the proton form factor measurements (where, admittedly, the statistical accuracy was much better than in the present case). They have found nominal bend angle uncertainties of $4.4 \mathrm{mrad}$ in the dispersive plane $\left(\Theta_{\text {bend }}\right)$, and $0.3 \mathrm{mrad}$ in the non-dispersive plane $\left(\Phi_{b e n d}\right)$. A full re-analysis with these offsets applied resulted in the following changes:

\begin{tabular}{|c|c|c|}
\hline & $\Delta K_{L T}$ & $\Delta K_{L L}$ \\
\hline$\Theta_{\text {bend }}(+4.4 \mathrm{mrad})$ & -0.002 & +0.000 \\
\hline$\Phi_{\text {bend }}(+0.3 \mathrm{mrad})$ & -0.001 & +0.000 \\
\hline
\end{tabular}

\section{Dilution Factor}

The method of fitting the $\delta x-\delta y$ spectra and obtaining the ratio of Compton events is sensitive to both the function used to fit the background distribution and to which plane the slices are taken. The systematic error on the dilution factor $(D)$, estimated by varying both of these factors, was found to be around +0.006 , which caused shifts of:

$$
\begin{aligned}
& \Delta K_{L T}=+0.004 \\
& \Delta K_{L L}=-0.001
\end{aligned}
$$

\section{$e p \gamma$ Correction}

The analysis described in sec. 5.4.2 has shown that while the $\pi^{0}$ background provides the dominant dilution of the RCS polarisation, a small but non-negligible dilution will occur due to the post-radiator bremsstrahlung $(e p \gamma)$ events. The ratio of the number of these events to the number of RCS events in the elliptical region of interest was found to be $2.4 \%$. Since the hard bremsstrahlung photons in this process carry almost all the initial electron polarisation (see Appendix B), it is sufficient within the accuracy of this calculation to use the values for elastic $e p$ polarisation transfer measured using the calibration data ${ }^{2}$ (and described in sec. 6.4). This correction, however, is based on a very limited data-set and the rather tenuous assumption that all those events around $\delta x=0$ in fig. 5.6 are due to the $e p \gamma$ process (when, clearly, some will be real Compton and other background events). Therefore, only half the correction is applied with a $100 \%$

\footnotetext{
${ }^{2}$ Transformation from the scattering plane to the CM frame gives $K_{L T}=0.189$ and $K_{L L}=$ 0.875 .
} 
systematic error:

$$
\begin{aligned}
& K_{L T}=0.111 \pm 0.001 \text { (ep } \gamma \text { syst) }
\end{aligned}
$$

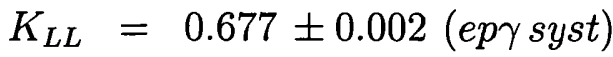

\subsection{1 $\pi^{0}$ Background Correction}

As alluded to earlier, the assumption of a constant background polarisation and the resulting correction is the largest source of systematic uncertainty in this measurement. However, we have already seen a piece of evidence that suggests the background correction is pretty self-consistent and the associated systematic uncertainties are smaller than the overall statistical error: the fact that the value of Compton polarisation is independent of the width of elliptical cut used to select Compton events (fig. 7.2). Even so, the inhomogeneity suggested by some of the reasonably large $\chi^{2}$ values in fig. 7.1 implies that propagation of the statistical errors associated with the background polarisation alone is not sufficient.

Perhaps the fact that we could have chosen any of the cut-widths in fig. 7.2 provides the most straightforward means by which to obtain an estimate of this systematic uncertainty. The difference between the polarisation transfer calculated for the particular elliptical region used in the final analysis and the value averaged over all of the seven regions has been found to be:

$$
\begin{aligned}
& \Delta K_{L T}=-0.032 \\
& \Delta K_{L L}=-0.032
\end{aligned}
$$

The observation that theses values are the same for both components is evidence, perhaps, for the consistency of this particular technique of estimation. In isolation, however, it is far from a rigorous analysis of the systematic uncertainty.

Two other techniques may be used to provide some further insight:

1. Examination of the variation of the polarisation result over four different rings around the centre of the Compton peak (from zero up to the maximum value of $1.6 n_{\text {sigma }}$ ), and the variation over the lower and upper halves of the calorimeter energy distribution. Such a study will provide clear evidence for deviations out with the statistical uncertainty. Fig. 7.3 clearly shows that no such deviations are present, but within the limited statistical accuracy it is difficult to use these plots for any reasonable quantitative estimation 

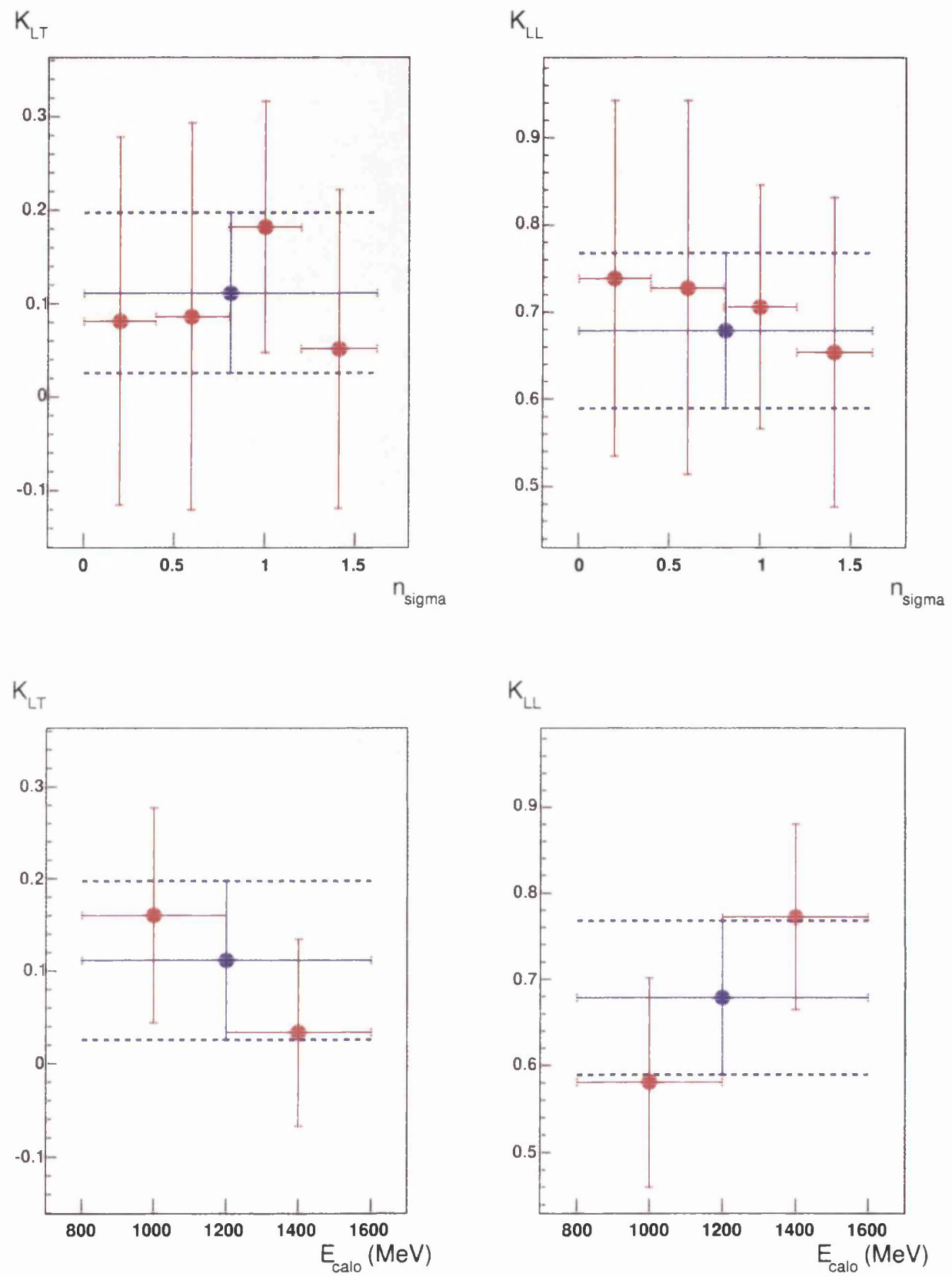

Figure 7.3: Variation in the $K_{L T}$ and $K_{L L}$ results for different cuts on $n_{\text {sigma }}$ (top) and $E_{\text {calo }}$ (bottom). The blue points show the final results; the red points correspond to more restrictive cuts. 
of the systematic uncertainty.

2. In looking at the stability plots of fig. 7.1, one might ask: why calculate $K^{\text {back }}$ over the full $\delta x-\delta y$ range when the primary interest lies at the centre of the distribution, and events in the periphery do not contribute much to the statistical accuracy? In an attempt to assess how large a difference might be expected if a more central $\delta x-\delta y$ background region is considered, the full calculation was repeated with the background components calculated for events satisfying the conditions, $a b s(\delta x)<20$ and $a b s(\delta y)<20$. The resultant changes in the Compton polarisation observables were found to be:

$$
\begin{aligned}
\Delta K_{L T} & =-0.023 \\
\Delta K_{L L} & =-0.016
\end{aligned}
$$

These shifts have the the same sign as our original estimates, but are significantly smaller in magnitude. Consequently, with some confidence we will use the more conservative estimate of -0.032 for the systematic uncertainty associated with the background correction.

\subsubsection{Total Systematic Uncertainty}

All of the contributions to the total systematic uncertainty which have been considered in the previous sections are included in fig. 7.4. As is usually the case with systematic uncertainties, it is difficult to say whether a linear or quadratic sum would best characterise the individual contributions and give an accurate estimate of the total. Since many of the above uncertainties are not symmetric, it seems reasonable to conclude that a linear sum would be the better option. The final results, therefore, including systematic uncertainties are:

$$
\begin{aligned}
& K_{L T}=0.111 \pm 0.078(\text { stat }) \pm 0.040 \text { (syst) } \\
& K_{L L}=0.677 \pm 0.083(\text { stat }) \pm 0.044 \text { (syst) }
\end{aligned}
$$



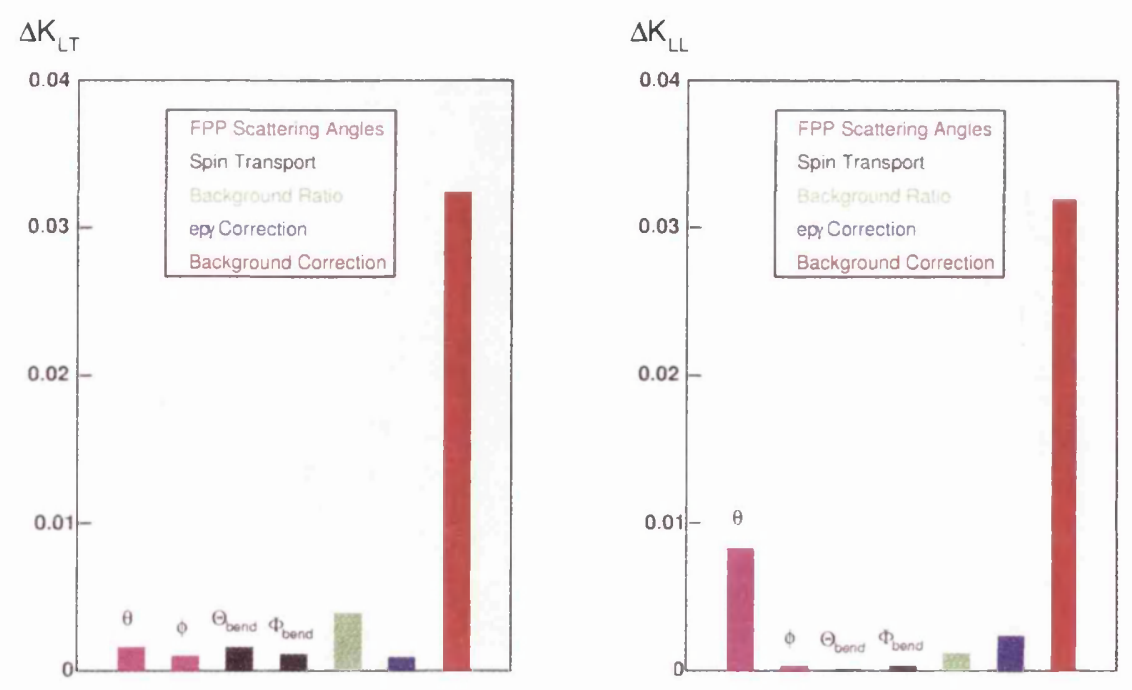

Figure 7.4: A comparison between all of the individual estimates of systematic uncertainty (absolute values) for $K_{L L}$ (left) and $K_{L T}$ (right). The $\pi^{0}$ background correction is the source of the largest uncertainty.

\subsection{Conclusions}

The primary motivation behind the first measurement of the proton spin observables in wide-angle Compton scattering was to determine the dominant reaction mechanism at presently accessible energies. Providing unambiguous resolution of this matter is important for two reasons:

1. The phenomenological analysis of exclusive processes at high momentum transfer has a distinctly reaction independent nature. Therefore, if one factorisation scheme dominates in a particular reaction channel, it might also be dominant in other similar reactions ${ }^{3}$.

2. With a particular theoretical approach favoured over the others, the full E99-114 data-set including the differential cross section results can be used to learn about the non-perturbative structure of the proton within that model (i.e. using a description based upon pQCD DA's or handbag Compton form factors).

\footnotetext{
${ }^{3}$ Such as deeply-virtual Compton scattering and hard meson production.
} 


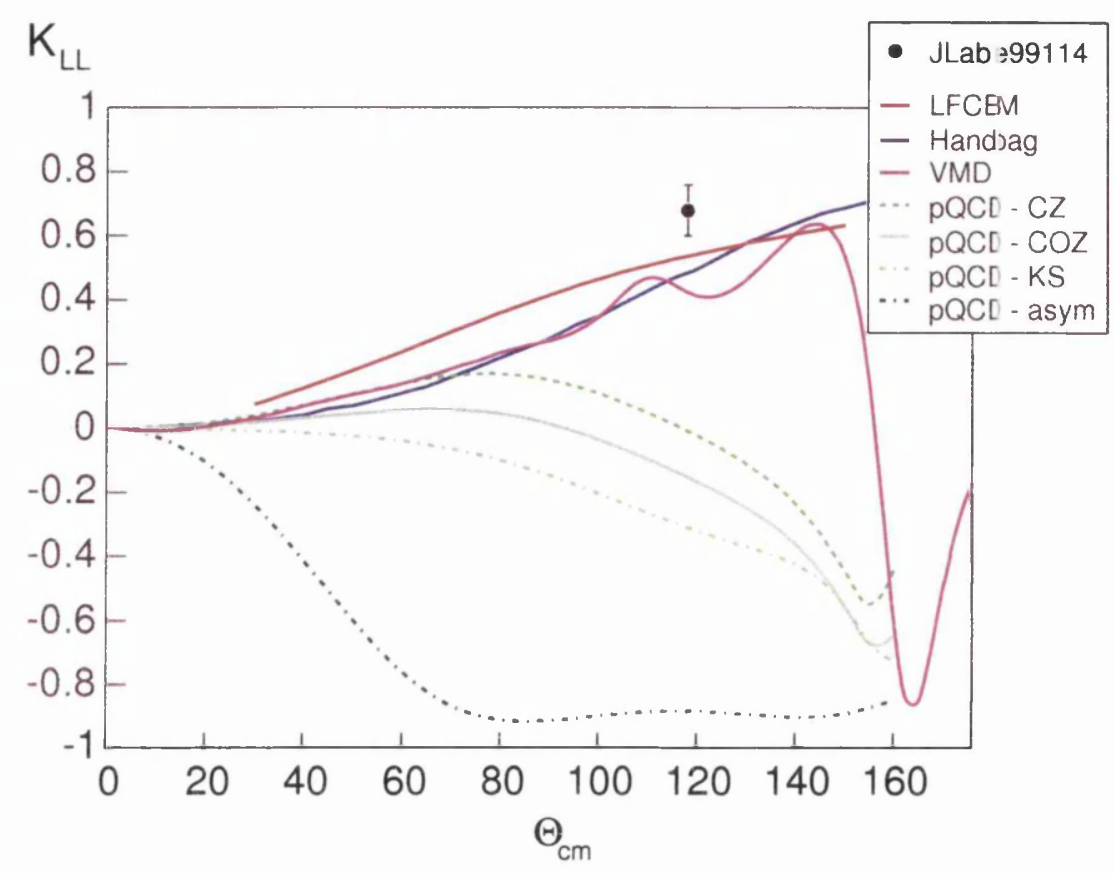

Figure 7.5: The present result and associated statistical uncertainty for the polarisation transfer, $K_{L L}$, relative to predictions from the $p Q C D$ (green), soft overlap (blue), LFCBM (red) and Regge exchange (magenta) calcultions.

\subsubsection{The Factorisation Scheme}

In the wide range of hard exclusive reactions none of the currently available data provides the opportunity for identification of the underlying factorisation scheme better than the measurement of the Compton scattering observable $K_{L L}$. This is because of the remarkable contrast between the $p Q C D$ leading twist and handbag mechanisms at large scattering angles. The present result unequirocally favours the latter of the two reaction mechanisms, and is shown relative to the various theoretical predictions discussed in Chapter 3 in fig. 7.5.

The overall uncertainty associated with the measurement is too large and the theoretical predictions too similar at these kinematics to distinguish between the soft overlap, LFCBM and Regge exchange predictions. One thing that is clear, however, is the unambiguous disagreement with the pQCD predictions, regardless of which proton DA's are used. This, in itself, is a powerful conclusion and adds to the body of evidence already in place highlighting the failure of pQCD predictions for some observables at JLab energies: the observation that the electromagnetic form factor ratio $F_{2} / F_{1}$ scales as $\Lambda / \sqrt{t}[33,34]$ and the non-zero value for the 
induced and transverse recoil proton polarisations in $\pi^{0}$ photoproduction [35] are direct evidence for non-zero proton helicity-flip. This means that nodels for the proton in this energy regime must involve configurations of the proton involving non-zero quark orbital angular momentum, which is not the case in all current pQCD calculations.

\subsubsection{Proton Structure within the Handbag Factorisation}

The Compton form factors and underlying GPD's of eq. 3.16 are a parameterisation of the process-independent, non-perturbative structure of the proton in the handbag approach. In sec. 3.3.1, $R_{A}$ and $R_{V}$ have been estimated using the overlap of soft light-cone wave functions, whereas an estimate for the parameter $\kappa_{T}$ (eq. 3.21) has been obtained from the assumption of a close relationship between the Compton form factor ratio $R_{T} / R_{V}$ and its electromagnetic counterpart $F_{2} / F_{1}$. These estimates have then been used to provide the soft overlap predictions for the cross section and spin observables shown in fig. 3.6.

Rather than relying on these estimates a model-independent determination of the Compton form factors is possible based upon the E99-114 data alone. A procedure for doing so has been suggested in ref. [37]. However, in addition to the experimental uncertainty associated with the present measurement, the architects of this approach have stressed that theoretical uncertainties at these energies are significant (they have been estimated to be around 15\%). As a result, the following discussion should not be considered a rigorous, quantitative analysis of the Compton form factors, but rather a discussion on what might be learned from this measurement and how it might be extended further.

\section{Proton helicity-flip and the magnitude of $\kappa_{T}$}

In the previous section we highlighted the necessity for including the contribution of proton helicity-flip amplitudes in any calculation of the experimental observables. Although the handbag predictions for the differential cross section are largely insensitive to the tensor form factor $\left(R_{T}\right)$ and the associated helicity-flip

amplitudes, this is not true for the polarisation transfer component $K_{L T}$. In eq. 3.21 we introduced the variable $\kappa_{T} \propto R_{T} / R_{V}$ as a measure of the difference between predictions with zero $\left(\kappa_{T}=0\right)$ and non-zero $\left(\kappa_{T}=0.37\right)$ proton helicityflip amplitudes. The $K_{L T}$ predictions for these two separate cases can be seen in fig. 7.6, together with the present result for this observable. 
One conclusion that can be made from inspection of fig 7.6 is that the magnitude of $\kappa_{T}$ lies somewhere between the values that have been considered, although the uncertainties are such that this is far from a robust statement. The authers of ref. [44] propose a method of obtaining a quantitative estimate for $\kappa_{T}$ by locking at the polarisation transfer ratio $\left(K_{L T} / K_{L L}\right)$. This ratio is relatively insensitive to the largest sources of theoretical uncertainty, and can be expressed by:

$$
\frac{K_{L T}}{K_{L L}} \simeq-\kappa_{T}(t) \frac{1-\beta \kappa_{T}^{-1}(t)}{1+\beta \kappa_{T}(t)}
$$

where

$$
\beta=\frac{2 m}{\sqrt{s}} \frac{\sqrt{-t}}{\sqrt{s}+\sqrt{-u}}=0.38,
$$

is calculated from the kinematic variables given in tab. 5.7. Using the present result:

$$
K_{L T} / K_{L L}=0.164 \pm 0.116(\text { stat })
$$

and solving eq. 7.2 gives the estimate:

$$
\kappa_{T}=\frac{\sqrt{-t}}{2 m} \frac{R_{T}}{R_{V}} \simeq 0.21 \pm 0.11 .
$$

Note that the error given for $\kappa_{T}$ is only that associated with the experimental statistical error. This result for $\kappa_{T}$ is more than one standard deviation smaller than the value of 0.37 predicted from the electromagnetic form factor ratio, which may suggest that $R_{T} / R_{V}$ falls more rapidly with $-t$ than $F_{2} / F_{1}$.

The relationship between $F_{2} / F_{1}$ and $R_{T} / R_{V}$ is based upon the equivalence of the underlying GPD's ( $E$ and $H$ ) at moderate momentum transfer, and illustrates why real Compton scattering is a natural complement to high $Q^{2}$ elastic $e p$ scattering. Polarised Compton scattering data, therefore, provides an ideal, independent means of resolving one of the most controversial issues in nucleon structure physics at present: the recent JLab polarisation result that $F_{2} / F_{1}$ scales as $\Lambda / \sqrt{-t}$ is in direct contradiction to Rosenbluth cross section data which scales as $\Lambda^{2} / t$ [94]. The scaling of the latter implies suppressed proton helicity-flip amplitudes, and therefore corresponds to small (close to zero) values of $\kappa_{T}$ at these energies. Some light may be shone on this apparent discrepancy by further measurements of $\kappa_{T}(t)$. 


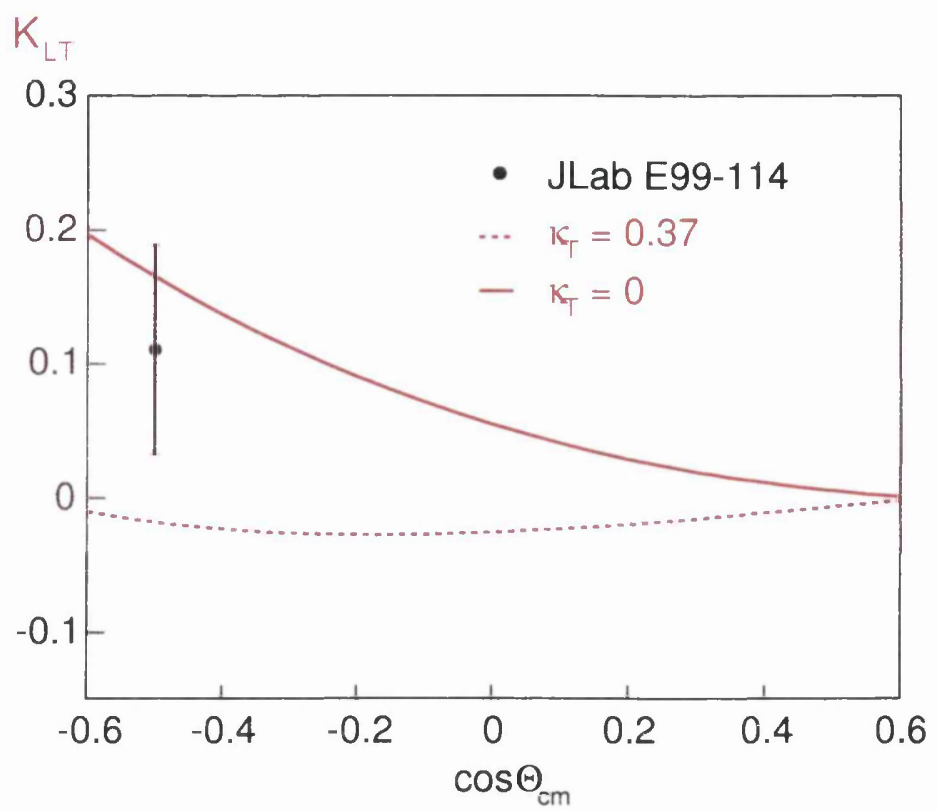

Figure 7.6: The present result and associated statistical uncertainty for the polarisation transfer, $K_{L T}$, compared with the soft overlap predictions for zero (solid) and non-zero (dashed) proton helicity-fiip amplitudes.

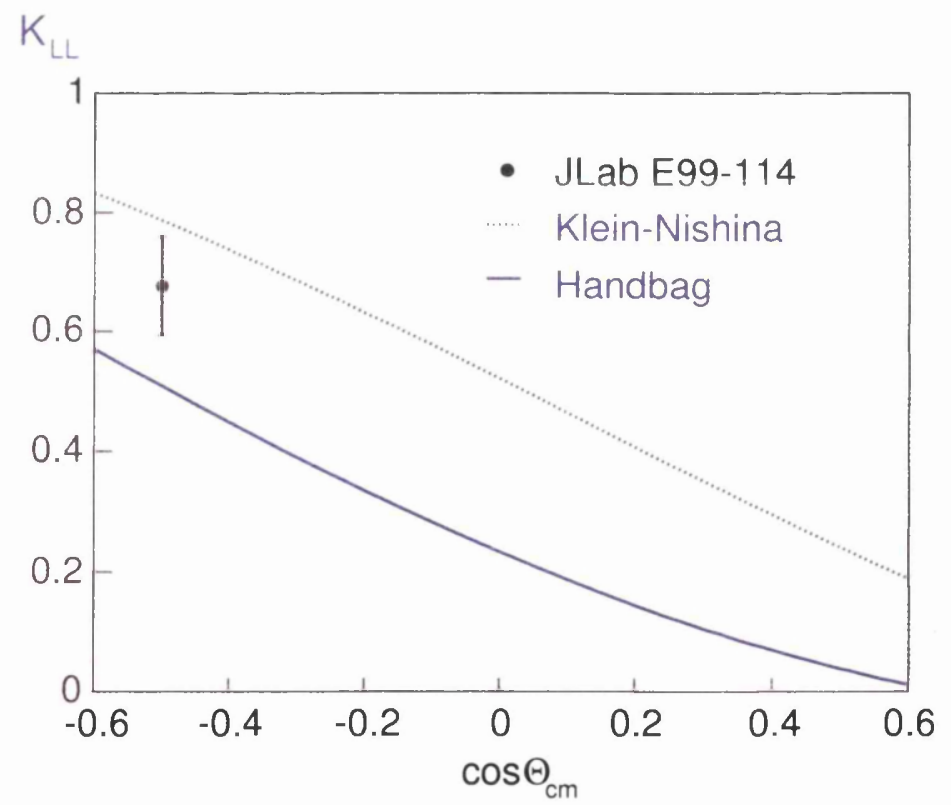

Figure 7.7: Comparison between the full soft overlap (solid) and Klein-Nishina subprocess (dotted) predcitions for $K_{L L}$. The present result and statistical uncertainty is also shown. 


\section{Comparison with the Klein-Nishina asymmetry: the ratio $R_{A} / R_{V}$}

The first obvious conclusion regarding $K_{L L}$ and the handbag calculations is that its positive nature implies that the active quark in the hard scattering subprocess is polarised in the same direction as the proton. Since the mass of the active quark is very small on the hadronic scale, not only will there be no quark helicity flip, almost all the quark helicity will be transferred to the parent hadron. The dominant contribution to $K_{L L}$, therefore, is from the hard $\gamma q$ subprocess, which is simply described by the Klein-Nishina asymmetry for the active structureless quark $\left(K_{L L}^{K N}\right)$. At leading order the subprocess amplitudes of eq. 3.15 give:

$$
K_{L L}^{K N}=\frac{\left|\mathcal{H}_{\uparrow \uparrow}^{\uparrow \uparrow}\right|^{2}-\left|\mathcal{H}_{\downarrow \uparrow}^{\uparrow \uparrow}\right|^{2}}{\left|\mathcal{H}_{\uparrow \uparrow}^{\uparrow}+\mathcal{H}_{\downarrow}^{\uparrow \uparrow}\right|^{2}}=\frac{s^{2}-u^{2}}{s^{2}+u^{2}}
$$

For the full handbag calculation of the physical amplitude, the $\Theta_{c m}$-dependence of $K_{L L}$ is essentially the same as for the subprocess, but the Compton form factors are responsible for a dilution in the overall magnitude (as seen in fig. 7.7). $K_{L L}$ is formally given by the equation:

$$
K_{L L} \simeq 2 K_{L L}^{K N} \frac{R_{A}(t)}{R_{V}(t)}\left(\frac{1+\beta \kappa_{T}(t)}{1+\kappa_{T}^{2}(t)}\right),
$$

where the values of $\beta$ and $\kappa_{T}$ determined in eq. 7.3 and 7.4 imply that the term within the brackets is very close to unity. The difference between the hard subprocess and full amplitude, therefore, is due almost exclusively to the ratio $R_{A} / R_{V}$. The comparison between the measured and predicted values for $K_{L L}$ is shown in fig. 7.7. The present result suggests that this form factor ratio may not be as large as expected, although unlike $K_{L T} / K_{L L}$ this observable is subject to significant theoretical corrections.

The form factor ratio $R_{A} / R_{V}$ is directly related to the DIS parton distribution ratio $\Delta q(x) / q(x)$ through eq. 3.20. Because the form factors are weighted by the square of the quark charge, the dominant contribution to the Compton form factors will be from valence $u$-quarks. Therefore, proton Compton scattering, in addition to all that has been discussed thus far, provides complementary measurements of the $u$-quark distribution within the proton. 


\subsubsection{Future Measurements}

The conclusions determined from the present result show some of the reasons why measurements involving proton Compton scattering, specifically the spin observables, are a powerful probe of proton structure. Two important questions remain, however, which must be answered in future experiments involving this reaction:

1. The soft overlap and LFCBM both involve the handbag factorisation, albeit with quite distinct models for the proton, whereas the Regge exchange amplitude is dominated by the hadronic component of the incoming and scattered photons. To test the validity of the Regge predictions, in addition to extending the kinematic range of measurements of the Compton form factors, JLab experiment E03-003 [95] will measure $K_{L L}$ and $K_{L T}$ for $\Theta_{c m}=$ $60^{\circ}, 100^{\circ}, 140^{\circ}$ and $160^{\circ}$.

2. It is a commonly held belief that the $\mathrm{pQCD}$ leading twist factorisation will dominate at sufficiently large momentum transfer, but at what energy scale does the transition to such a domain occur? Furthermore, are there some intermediate dominant factorisation schemes (perhaps involving a single hard gluon exchange)? The answer to these questions will only become clear at much higher energies than those currently available $(\sim 50-100 \mathrm{GeV})$. 


\section{Appendix A}

\section{Kinematic Calculations}

Compton scattering, elastic ep scattering and coherent $\pi^{0}$ photo-production are described by simple two-body kinematics. By detecting the recoil proton in coincidence with an electromagnetic shower in the calorimeter, the four-momentum of the proton and the mass of its scattered partner uniquely define the incident particle energy. Conservation of energy and momentum can then be applied to determine the scattered particle momentum.

\section{Coordinate Systems}

The key task in the present analysis is to correlate events at the calorimeter with the proton kinematic quantities reconstructed by the HRS. For the associated calculations, it is important to be aware of three distinct coordinate systems and the relationships between them. The experimental geometry is shown in fig. A.1. All three systems have their origin at the Hall A central pivot, and the HRS (which rotates about the pivot) is located on the left-hand side of the hall, with the calorimeter on the right-hand side.

In the $\boldsymbol{H a l l}$ coordinate system, $\mathbf{z}_{\text {hall }}$ points along the beam direction, $\mathbf{x}_{\text {hall }}$ points to the left and $\mathbf{y}_{\text {hall }}$ points vertically up. The $\boldsymbol{H} \boldsymbol{R} \boldsymbol{S}$ coordinate system, which is equivalent to the transport system (on an event-by-event basis, as described in sec. 5.2.2), has $\mathbf{z}_{\text {tgt }}$ pointing along the spectrometer axis, $\mathbf{x}_{\text {tgt }}$ pointing vertically down and $\mathbf{y}_{\text {tgt }}=\mathbf{z}_{\text {tgt }} \times \mathbf{x}_{\text {tgt }}$. Finally, the Calorimeter coordinate system is defined such that $\mathbf{z}_{\mathbf{c a l o}}$ points from the target (pivot point) to the centre of the calorimeter. Looking along this axis, $\mathbf{x}_{\text {calo }}$ then points to the left and $\mathbf{y}_{\text {calo }}$ points up.

A further three quantities are required for kinematic calculations involving 


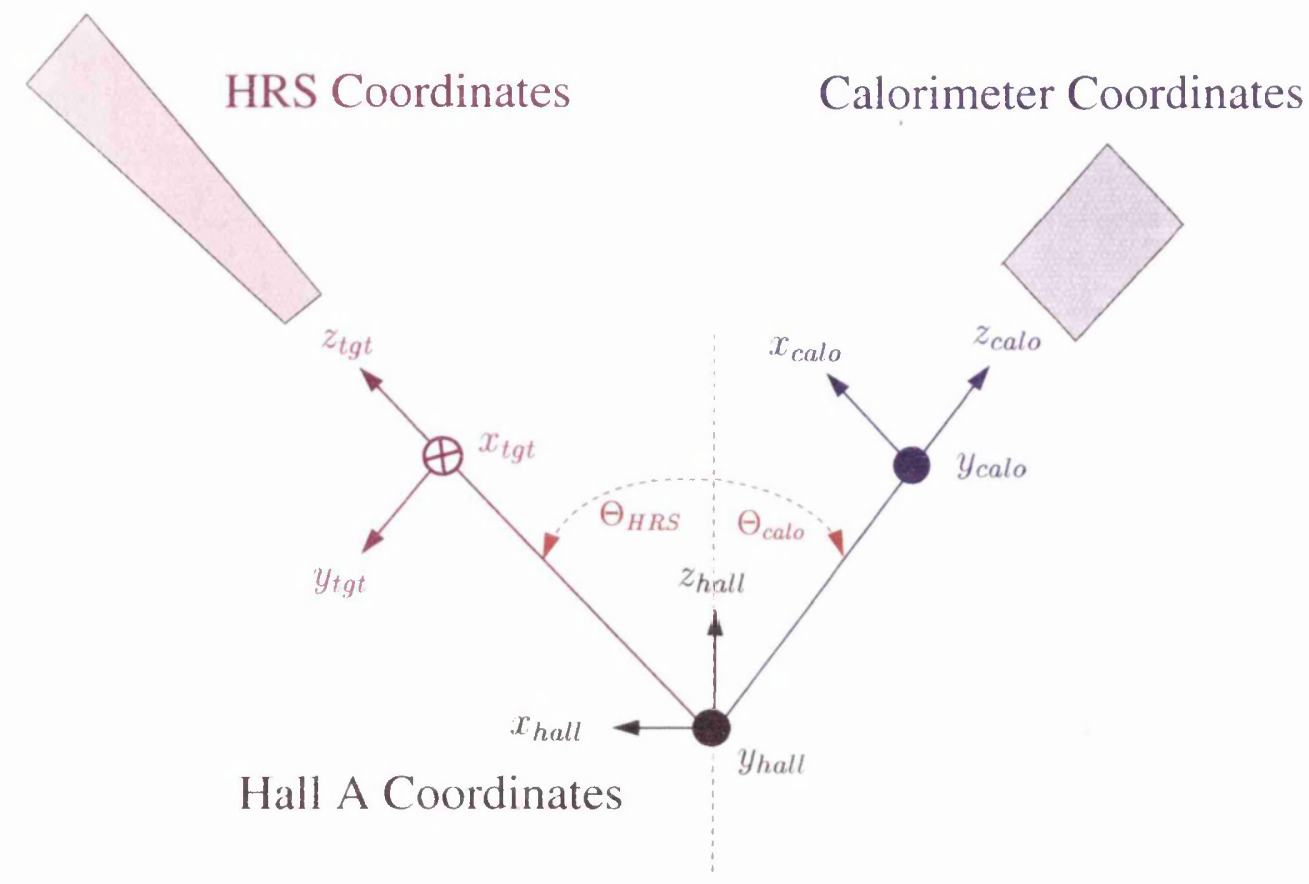

Figure A.1: Definitions of the three coordinate systems used in the kinematic calculations of this appendix: the Hall (black), HRS (magneta) and Calorimeter (blue) coordinate systems.

these coordinate systems:

1. The distance between the hall central pivot and the front face of the calorimeter $\left(D_{\text {calo }}^{\prime}\right)$.

2. The angle $\mathbf{z}_{\text {calo }}$ makes with respect to the $z_{\text {hall }}$-axis $\left(\Theta_{\text {calo }}\right)$.

3. The equivalent on the other side of the beamline $\left(\Theta_{H R S}\right)$.

The transformations between the coordinate systems can then be defined by the rotation matrices:

From Hall to $\boldsymbol{H R} \boldsymbol{S}$ coordinates:

$$
\left[\begin{array}{l}
x \\
y \\
z
\end{array}\right]_{t g t}=\left[\begin{array}{ccc}
0 & -1 & 0 \\
\cos \Theta_{H R S} & 0 & -\sin \Theta_{H R S} \\
\sin \Theta_{H R S} & 0 & \cos \Theta_{H R S}
\end{array}\right]\left[\begin{array}{l}
x \\
y \\
z
\end{array}\right]_{\text {hall }} .
$$


From Hall to Calorimeter coordinates:

$$
\left[\begin{array}{l}
x \\
y \\
z
\end{array}\right]_{\text {calo }}=\left[\begin{array}{ccc}
\cos \Theta_{\text {calo }} & 0 & \sin \Theta_{\text {calo }} \\
0 & 1 & 0 \\
-\sin \Theta_{\text {calo }} & 0 & \cos \Theta_{\text {calo }}
\end{array}\right]\left[\begin{array}{l}
x \\
y \\
z
\end{array}\right]_{\text {hall }} .
$$

In addition, the target position along the $z_{\text {hall }}$-axis corresponding to the physical event vertex is required to calculate the distance between this vertex point and calorimeter, as well as the associated $x$-offset on the calorimeter face. If the vertex point is $z_{v}$, the distance and $x$-offset are given by:

$$
\begin{aligned}
D_{\text {calo }} & =D_{\text {calo }}^{\prime}-z_{v} \cos \Theta_{\text {calo }} \\
x_{0} & =z_{v} \sin \Theta_{\text {calo }} .
\end{aligned}
$$

\section{Reconstruction of Proton Kinematic Quantities}

The proton parameters measured and reconstructed in espace are $\theta_{t g t}, y_{t g t}, \phi_{t g t}$ and $p_{h r s}$ (from $\delta$ and the central proton momentum). We begin by calculating the angle the proton trajectory makes with the central $z_{H R S}$-axis:

$$
\cos \theta_{h r s}^{z}=\frac{1}{\sqrt{1+\tan ^{2} \theta_{t g t}+\tan ^{2} \phi_{t g t}}} .
$$

This leads to the proton momentum in the $\boldsymbol{H R S}$ coordinate system:

$$
\mathbf{p}_{\mathrm{hrs}}=\left(\begin{array}{c}
p_{h r s} \cos \theta_{z}^{h r s} \tan \theta_{t g t} \\
p_{h r s} \cos \theta_{z}^{h r s} \tan \phi_{t g t} \\
p_{h r s} \cos \theta_{z}^{h r s}
\end{array}\right)
$$

which can then be transformed into the $\boldsymbol{H a l l}$ coordinate system by the rotation defined by the inverse of eq. A.1. This leaves us finally with the proton fourmomentum, from which we can calculate the proton scattering angle relative to the $z_{\text {hall }}$-axis:

$$
\cos \theta_{z}=\frac{p_{z}}{p}
$$




\section{Two-body Kinematic Correlation}

To find the four-momentum of the scattered particle which induces a calorimeter trigger an elastic two-body reaction is assumed:

$$
\gamma+p \rightarrow p+X
$$

where $X=\gamma, e^{-}, \pi^{0}$ are the dominant reactions of interest. It is further assumed that there is no kinematic distinction between an incident photon and incident electron (i.e. we neglect the electron mass in these calculations). Therefore, using eq. A.7, the energy of the incident photon/electron can be determined:

$$
E_{\text {in }}=\frac{M_{p}\left(E_{p}-M_{p}\right)+\frac{1}{2} M_{X}^{2}}{M_{p}-E_{p}+p \cos \theta_{z}} .
$$

By four-momentum conservation the energy, three-momentum and in-plane scattering angle of the particle $X$ are given by:

$$
\begin{aligned}
E_{X} & =E_{i n}+M_{p}-E_{p} \\
k & =\sqrt{E_{X}^{2}-M_{X}^{2}} \\
\cos \theta_{X} & =\frac{E_{i n} E_{X}-M_{p}\left(E_{p}-M_{p}\right)-\frac{1}{2} M_{X}^{2}}{E_{i n} k} .
\end{aligned}
$$

Moreover, by conservation of out-of-plane momentum the azimuthal angle can be found:

$$
\sin \phi_{X}=-\frac{p_{y}}{k \sin \theta_{X}}
$$

This yields the momentum of the proton's partner in the Hall frame:

$$
\mathbf{k}=\left(\begin{array}{c}
-k \sin \theta_{X} \cos \phi_{X} \\
k \sin \theta_{X} \sin \phi_{X} \\
k \cos \theta_{X}
\end{array}\right)
$$

\section{Predicted Calorimeter Hit Position}

The knowledge of this particle's four-momentum allows calculation of the position where it will strike the calorimeter face. It's momentum is rotated to the Calorimeter coordinate system by application of eq. A.2, resulting in the vector 


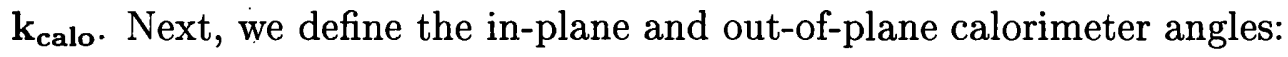

$$
\begin{aligned}
\tan \theta_{\text {calo }} & =\frac{k_{\text {calo }}^{x}}{k_{\text {calo }}^{z}} \\
\tan \phi_{\text {calo }} & =\frac{k_{\text {calo }}^{y}}{k_{\text {calo }}^{z}}
\end{aligned}
$$

Using these angles and the relations in eq. A.3 and A.4, the predicted $x$ and $y$ position of the calorimeter hit will be:

$$
\begin{aligned}
& x_{\text {predicted }}=x_{0}+D_{\text {calo }} \theta_{\text {calo }}, \\
& y_{\text {predicted }}=D_{\text {calo }} \phi_{\text {calo }} .
\end{aligned}
$$

Finally, we come to the most important of the kinematic variables, the difference between the predicted and measured hit postions:

$$
\begin{aligned}
\delta x & =x_{\text {predicted }}-x_{\text {grav }} \\
\delta y & =y_{\text {predicted }}-y_{\text {grav }}
\end{aligned}
$$




\section{Appendix B}

\section{Beam Polarisation}

Only one measurement of the longitudinal polarisation of the electron beam was taken using the Møller polarimeter (sec. 4.2.1). This occurred at the beginning of the polarised data-taking period. Assuming no large deviations from this measured value over relatively short time periods, which is consistent with the behaviour of the Jlab polarised electron source, the asymmetries measured during the experiment can be used as a de facto monitor of the stability of beam polarisation. Since the physical proton polarisation transfer components for a particular primary reaction at the target (identified by the means described in sec. 5.4) do not vary with time, any deviation in the resultant asymmetries (out with statistical fluctuations) will be clear evidence for variation in the beam polarisation. Clearly, the abundance of the $\pi^{0}$ background events in the production data-set makes the asymmetries corresponding to these events ideal for such a study.

However, the electron polarisation is not the appropriate quantity for obtaining the RCS polarisation transfer components; rather, it is the polarisation of the incident bremsstrahlung photons that is required. The transfer of polarisation from electron to photon in the bremsstrahlung process has been calculated in ref. [96]. It turns out the ratio of photon to electron polarisation depends only on the ratio of the corresponding energies, according to the polynomial [58]:

$$
\frac{P_{\gamma}}{P_{e}}=\frac{4 \frac{E_{\gamma}}{E_{e}}-\left(\frac{E_{\gamma}}{E_{e}}\right)^{2}}{4-4 \frac{E_{\gamma}}{E_{e}}+3\left(\frac{E_{\gamma}}{E_{e}}\right)^{2}} .
$$

Since the incident photon energy is spread out over a range of values (see fig. 5.4) 
the correction of eq. B.1 must be applied on an event-by-event basis. The resultant polarisation spread over the range of incident energies was found to be 98.2 $99.8 \%$ (FWHM) of the incident electron polarisation, and the average value was $99.2 \%$.

To examine the photon beam stability the $\pi^{0}$ background polarisation transfer components $\left(K^{b a c k}\right)$, uncorrected for the beam polarisation $\left(P_{\gamma}\right)$, have been calculated as a function of the time elapsed since the Møller measurement. The results can be seen in the top two plots in fig. B.1. To isolate the relative variation in the photon beam polarisation $\left(\Delta P_{\gamma}\right)$, which is shown in the bottom plot of fig. B.1, one must normalise these quantities (e.g. $P_{\gamma} K_{L T}^{b a c k} / K_{L T}^{\hat{b a c k}}$ ) and take the average of the two. Within the statistical accuracy of the measurement the results appear to be very stable. The maximum drift in the polarisation from the start to the end of the experimental period (obtained by a straight line fit to the data in fig. B.1) was found to be around $3 \%$. This is actually smaller than the relative uncertainty associated with the Møller measurement $\left(P_{e}=0.766 \pm 0.026\right)$.

Therefore, in all the polarisation analysis that is described throughout this document we assume that the value and uncertainty for the electron beam polarisation is given by the result of Møller measurement, and the corresponding photon polarisation for a particular event is calculated from eq. B.1. 

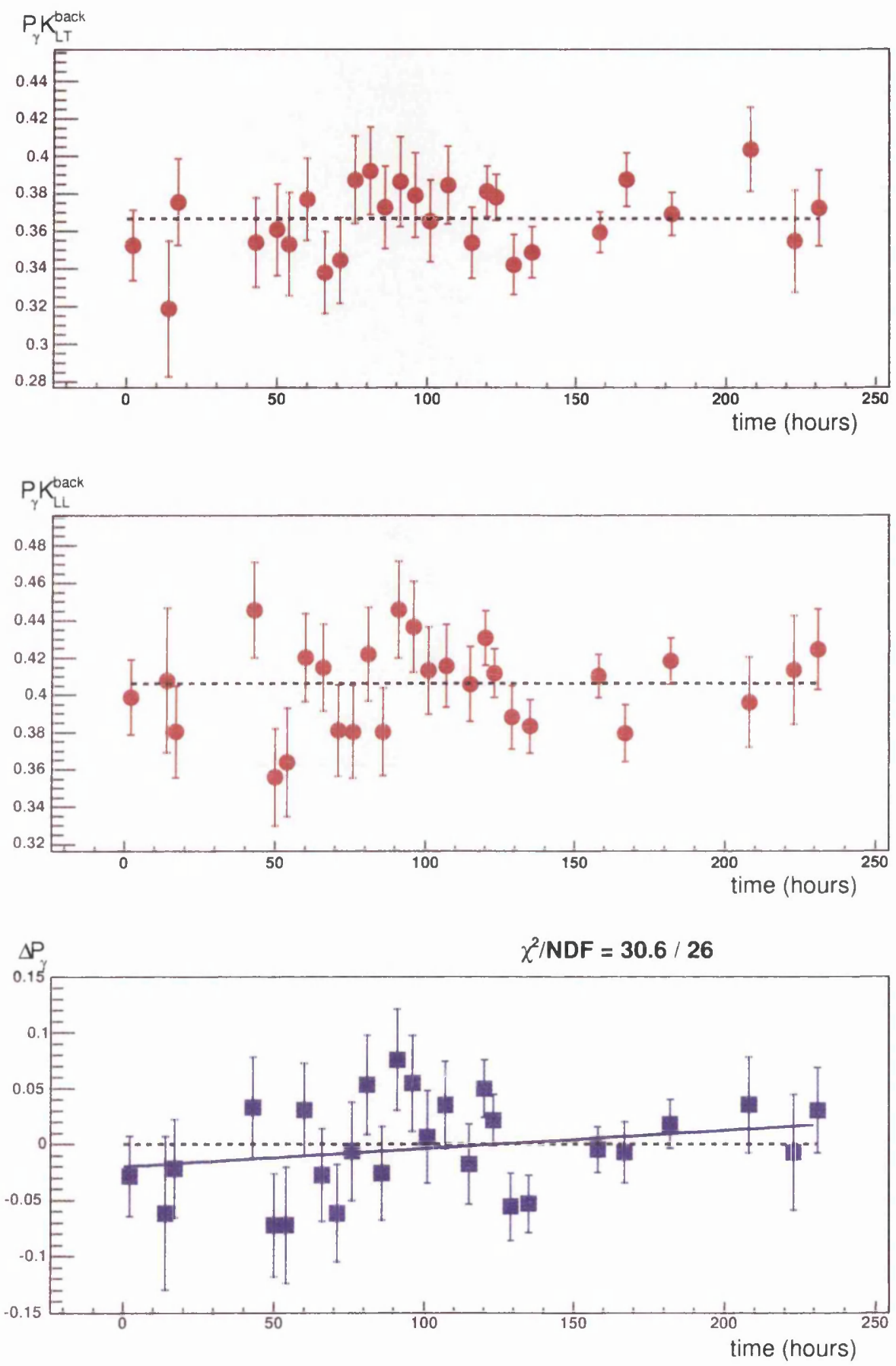

Figure B.1: Plots showing the variation of the beam polarisation over time. The data in red show the variation of the background polarisation transfer components, $P_{\gamma} K_{L T}^{\text {back }}$ (top) and $P_{\gamma} K_{L L}^{\text {back }}$ (middle). The blue data shows the relative shift in the averaged values after normalisation (bottom). 


\section{Bibliography}

[1] A.W. Thomas and W. Weise. The structure of the nucleon. Berlin, Germany: Wiley-VCH (2001).

[2] R. Hofstadter and R.W. McAllister. Electron scattering from the proton. Phys. Rev., 98:217-218, 1955.

[3] P. Mergeil, U.G. Meissner and D. Drechsel. Dispersion theoretical analysis of the nucleon electromagnetic form-factors. Nucl. Phys., A596:367-396, 1996. (hep-ph/9506375).

[4] M. Breidenbach et al. Observed behavior of highly inelatsic electron-proton scattering. Phys. Rev. Lett., 23:935-939, 1969.

[5] J.D. Bjorken. Asymptotic sum rules at infinite momentum. Phys. Rev., 179:1547-1553, 1969.

[6] R.G. Roberts. The structure of the proton. Cambridge, UK: Cambridge University Press (1990).

[7] J. Ashman et al. An investigation of the spin structure of the proton in deep inelastic scattering of polarized muons on polarized protons. Nucl. Phys., B 328:1, 1989.

[8] K. Goeke, M.V. Polyakov and M. Vanderhaeghen. Hard exclusive reactions and the structure of hadrons. Prog. Part. Nucl. Phys., 47:401-515, 2001. (hep-ph/0106012).

[9] M. Diehl. Generalized parton distributions. Phys. Rept., 388:41-277, 2003. (hep-ph/0307382).

[10] G.P. Lepage and S.J. Brodsky. Exclusive processes in perturbative quantum chromodynamics. Phys. Rev., D 22:2157, 1980. 
[11] X. Ji. Gauge-invariant decomposition of nucleon spin. Phys. Rev. Lett., 78:610, 1997. (hep-ph/9604329).

[12] O. Klein and Y. Nishina. On the scattering of radiation off free electrons according to the new relativistic quantum dynamics of Dirac. Z. Phys., 52:853, 1929.

[13] D. Drechsel, B. Pasquini and M. Vanderhaeghen. Dispersion relations in real and virtual Compton scattering. Phys. Rept., 378:99-205, 2003. (hep-ph/0212124).

[14] A.M. Baldin. Polarizibility of nucleons. Nucl. Phys., 18:310, 1960.

[15] D. Babusci, G. Giordano and G. Matone. A new evaluation of the Baldin sum rule. Phys. Rev., C 57:291-294, 1998. (nucl-th/9710017).

[16] S. Gerasimov. Sov. J. Nucl. Phys., 2:430, 1966.

[17] S.D. Drell and A.C. Hearn. Exact sum rule for nucleon magnetic moments. Phys. Rev. Lett., 16:908, 1966.

[18] S.J. Brodsky and G.P. Lepage. Helicity selection rules and tests of gluon spin in exclusive QCD processes. Phys. Rev., D 24:2848, 1981.

[19] S.J. Brodsky and G.R. Farrar. Scaling laws for large momentum transfer processes. Phys. Rev., D 11:1309, 1975.

[20] E. Maina and G.R. Farrar. Large angle Compton scattering in perturbative QCD. Phys. Lett., B 206:120, 1988.

[21] G.R. Farrar, G. Sterman and H. Zhang. Absence of Sudakov factors in large angle photoproduction and Compton scattering. Phys. Rev. Lett., 62:2229, 1989.

[22] G.R. Farrar and H. Zhang. Perturbative QCD calculation of real and virtual Compton scattering. Phys. Rev., D 41:3348-3359, 1990.

[23] A.S. Kronfeld and B. Nizic. Nucleon Compton scattering in perturbative QCD. Phys. Rev., D 44:3445-3465, 1991.

[24] M. Vanderhaeghen, P.A.M Guichon and J. Van de Wiele. Compton scattering and the nucleon valence wavefunction. Nucl. Phys., A 622:144c-156c, 1997. 
[25] T.C. Brooks and L.J. Dixon. Recalculation of proton Compton scattering in perturbative QCD. Phys. Rev., D 62:114021, 2000. (hep-ph/0004143).

[26] V.L. Chernyak and I.R. Zhitnitsky. Asymptotic behavior of exclusive processes in QCD. Phys. Rept., 112:173, 1984.

[27] I.D. King and C.T. Sachrajda. Nucleon wave functions and QCD sum rules. Nucl. Phys., B 279:785, 1987.

[28] V.L. Chernyak, A.A. Ogloblin and I.R. Zhitnitsky. The wave functions of the octet baryons. Z. Phys., C 42:569, 1989.

[29] N. Isgur and C.H. Llewellyn Smith. The applicability of perturbative QCD to exclusive processes. Nucl. Phys., B 317:526-572, 1989.

[30] M.A. Shupe et al. Neutral pion photoproduction and proton Compton scattering at large angles. Phys. Rev., D 19:1921-1930, 1979.

[31] P.R. Cameron et al. Measurement of the analyzing power for $p+p_{\uparrow} \rightarrow p+p$ at $p_{\perp}^{2}=6.5(\mathrm{GeV} / \mathrm{c})^{2}$. Phys. Rev., D 32:3070, 1985.

[32] T. Feldmann and P. Kroll. Implications of light-quark admixtures on charmonium decays into meson pairs. Phys. Rev., D 62:074006, 2000. (hep-ph/0003096).

[33] M.K. Jones et al. $G_{E_{p}} / G_{M_{p}}$ ratio by polarization transfer in $\vec{e}+p \rightarrow e+\vec{p}$. Phys. Rev. Lett., 84:1398-1402, 2000. (nucl-ex/9910005).

[34] O. Gayou et al. Measurement of $G_{E_{p}} / G_{M_{p}}$ in $\vec{e}+p \rightarrow e+\vec{p}$ to $Q^{2}=5.6 \mathrm{GeV}^{2}$. Phys. Rev. Lett., 88:092301, 2002. (nucl-ex/0111010).

[35] K. Wijesooriya et al. Polarization measurements in neutral pion photoproduction. Phys. Rev., C 66:034614, 2002.

[36] C. Bourrely, J. Soffer and E. Leader. Polarization phenomena in hadronic reactions. Phys. Rept., 59:95-297, 1980.

[37] H.W. Huang, P. Kroll and T. Morii. Perturbative and non-perturbative QCD corrections to wide-angle Compton scattering. Eur. Phys. J., C 23:301-310, 2002. (hep-ph/0110208). 
[38] J.D. Bjorken and E.A. Paschos. Inelastic electron proton and gamma proton scattering, and the structure of the nucleon. Phys. Rev., 185:1975-1982, 1969.

[39] H.W. Huang and P. Kroll. Large momentum transfer electroproduction of mesons. Eur. Phys. J., C 17:423-435, 2000. (hep-ph/0005318).

[40] M. Diehl, P. Kroll and C. Vogt. Two-photon annihilation into baryonantibaryon pairs. Eur. Phys. J., C 26:567-577, 2003. (hep-ph/0206288).

[41] A.V. Radyushkin. Nonforward parton densities and soft mechanism for form factors and wide-angle Compton scattering in QCD. Phys. Rev., D 58:114008, 1998. (hep-ph/9803316).

[42] M. Diehl, T. Feldmann, R. Jakob and P. Kroll. Linking parton distributions to form factors and Compton scattering. Eur. Phys. J., C 8:409-434, 1999. (hep-ph/9811253).

[43] M. Diehl, T. Feldmann, R. Jakob and P. Kroll. Skewed parton distributions in real and virtual Compton scattering. Phys. Lett., B 460:204-212, 1999. (hep-ph/9903268).

[44] M. Diehl, T. Feldmann, H.W. Huang and P. Kroll. Proton mass effects in wide-angle Compton scattering. Phys. Rev., D 67:037502, 2003. (hep-ph/0212138).

[45] M. Diehl, T. Feldmann, R. Jakob and P. Kroll. The overlap representation of skewed quark and gluon distributions. Nucl. Phys., B 596:33-65, 2001. (hep-ph/0009255).

[46] M. Gluck, E. Reya and A. Vogt. Dynamical parton distributions revisited. Eur. Phys. J., C 5:461-470, 1998. (hep-ph/9806404).

[47] H.W. Huang (U. Colorado). Private Communication.

[48] G.A. Miller and M.R. Frank. $Q^{2}$ indepénence of $Q F_{2} / F_{1}$, Poincaré invariance and the non-conservation of helicity. Phys. Rev., C 65:065205, 2002. (nuclth/0201021).

[49] G.A. Miller. Light front cloudy bag model: Nucleon electromagnetic form factors. Phys. Rev., C 66:032201, 2002. (nucl-th/0207007). 
[50] A. Chodos, R.L. Jaffe, K. Johnson and C.B. Thorn. Baryon structure in the bag theory. Phys. Rev., D 10:2599, 1974.

[51] A.W. Thomas, S. Theberge and G.A. Miller. The cloudy lag model of the nucleon. Phys. Rev., D 24:216, 1981.

[52] G.A. Miller. Handling the handbag diagram in Compton scattering on the proton. Phys. Rev., C 69:052201, 2004. (nucl-th/0402092).

[53] G.A. Miller (U. Washington). Private Communication.

[54] T.H. Bauer, R.D. Spital, D.R. Yennie and F.M. Pipkin. The hadronic properties of the photon in high-energy interactions. Rev. Mod. Phys., 50:261, 1978.

[55] F. Cano and J.M. Laget. Real and virtual Compton scattering in a Regge approach. Phys. Lett., B 551:317-323, 2003. (hep-ph/0209362).

[56] F. Cano and J.M. Laget. Compton scattering, vector meson photoproduction and the partonic structure of the nucleon. Phys. Rev., D 65:074022, 2002. (hep-ph/0111146).

[57] J.R. Fornshaw and D.A. Ross. Quantum chromodynaimcs and the Pomeron. Cambridge, UK: Cambridge University Press (1997).

[58] C. Hyde-Wright, A.M. Nathan and B. Wojtsekhowski. Exclusive Compton scattering on the proton. JLab Proposal, E99-114.

[59] C.W. Leemann, D.R. Douglas and G.A. Krafft. The Continuous Electron Beam Accelerator Facility: CEBAF at the Jefferson Laboratory. Ann. Rev. Nucl. Part. Sci., 51:413-450, 2001.

[60] J. Alcorn et al. Basic instrumentation for Hall $A$ at Jefferson Lab. Nucl. Instrum. Meth., A 522:294-346, 2004.

[61] E.J. Brash, R.D. Ransome and C.W. deJager. Hall A Status Report 1999.

[62] The Hall A Møller polarimeter website. http://www.jlab.org/ moller.

[63] The Hall A cryotraget, system website. http://hallaweb.jlab.org/equipment/targets/cryotargets/Halla_tgt.html. 
[64] J.L. Matthews and R.O. Owens. Accurate formulae for the calculation of high energy electron bremsstrahlung spectra. Nucl. Instrum. Meth., A 111:157, 1973.

[65] K.G. Fissum et al. Vertical drift chambers for the Hall A high resolution spectrometers at Jefferson Lab. Nucl. Instrum. Meth., A 474:108-131, 2001.

[66] L. Bimbot et al. A focal plane polarimeter for protons with kinetic energies up to $1.85 \mathrm{GeV}$. Nucl. Instrum. Meth. (in preparation), available at:http://hallaweb.jlab.org/equipment/detectors.fpp.html.

[67] B.D. Milbrath et al. A comparison of polarization observables in electron scattering from the proton and deuteron. Phys. Rev. Lett., 80:452-455, 1998. (nucl-ex/9712006).

[68] T. Pospischil et al. The horizontal drift chambers for the focal plane proton polarimeter of the 3-spectrometer setup at MAMI. Nucl. Instrum. Meth., A 483:726-733, 2002.

[69] T.N. Taddeucci et al. A neutron polarimeter for $(p, n)$ measurements at intermediate energies. Nucl. Instrum. Meth., A 241:448-460, 1985.

[70] E. Brash, M.K. Jones, C.F. Perdrisat and V. Punjabi. Measurement of $G_{E_{p}} / G_{M_{p}}$ to $Q^{2}=5.6 \mathrm{GeV}^{2}$ by the recoil polarization method. JLab Proposal, E99-007.

[71] O. Gayou. Measurement of proton form factors ratio $G_{E_{p}} / G_{M_{p}}$ to $Q^{2}=$ $5.6 \mathrm{GeV}^{2}$ by recoil polarimtery. $\mathrm{PhD}$ thesis, College of William and Mary, VA, 2002.

[72] L.S. Azhgirei et al. Measurement of analyzing powers for polarized proton scattering on $\mathrm{CH}_{2}$ target for proton momentum range from $1.75 \mathrm{GeV} / \mathrm{c}$ to $5.3 \mathrm{GeV} / \mathrm{c}$. AIP Conf. Proc., 675:826-829, 2003.

[73] J.P. Chen et al. Study of Hall A photon spectrometer. JLab Internal Report, 1999.

[74] V. Mamyan (Yerevan) and V. Nelyubin (St. Petersburg). Private Communication.

[75] G. Heyes et al. The CEBAF online data acquisition system. Given at Computing in High-energy Physics (CHEP 94), San Francisco, CA, 1994. 
[76] E. Jastrzembski et al. The Jefferson Lab Trigger Supervisor System. Given at the 11th IEEE NPSS Real Time Conference, Santa Fe, NM, 1999.

[77] E. Offermann. ESAPCE Users Guide. JLab Internal Report, 1997.

[78] Pro Fortran User Guide. www.absoft.com/literature/inteluserguide.pdf.

[79] CERN Program Library.

http://wwwinfor.cern.ch/asdoc/kuip/kuipoverview.html.

[80] N. Liyanage. Optics calibration of the Hall A high resolution spectrometers. JLab Technical Note, TN 02-012.

[81] The Hall A ROOT project website. http://hallaweb.jlab.org/root.

[82] R. Brun and F. Rademakers. ROOT: An object oriented data analysis framework. Nucl. Instrum. Meth., A 389:81-86, 1997.

[83] E. Voutier and C.W. deJager. Hall A Status Report 2002.

[84] S. Strauch (GWU). Private Communication.

[85] W.H. Press, B.P. Flannery, S.A. Teukolsky and W.T. Vetterling. Numerical Recipes in FORTRAN: The Art of Scientific Computing. Cambridge, UK: Cambridge University Press (1989).

[86] K. Makino and M. Berz. COSY INFINITY version 8. Nucl. Instrum. Meth., A 427:338-343, 1999.

[87] L. Pentchev and J.J. Lerose. Quadrupole alignment studies in the HRSe. JLab Technical Note, TN 01-052.

[88] L. Pentchev. Spin transport in the HRSe. JLab Technical Note, TN 04-012.

[89] R. Hagedorn. Relativistic kinematics. New York, USA: WA Benjamin (1964).

[90] V. Dmitrasinovic. Wigner-Thomas spin precession in polarized coincidence electronuclear scattering. Phys. Rev., C 47:2195, 1993.

[91] D. Bessetet al. A set of efficient estimators for polarization measurements. Nucl. Instrum. Meth., A 166:515, 1979.

[92] C.F. Perdrisat and V. Punjabi. Electric form factor of the proton by recoil polarimetry. JLab Proposal, E93-027. 
[93] M.K. Jones (Jlab). Private Communication.

[94] J. Arrington. Implications of the discrepancy between proton form factor measurements. Phys. Rev., C 69:022201, 2004. (nucl-ex/0309011).

[95] A.M. Nathan and B. Wojtsekhowski. Polarization transfer in wide angle Compton scattering. JLab Proposal, E99-114.

[96] H. Olsen and L.C. Maximon. Photon and electron polarization in high-energy bremsstrahlung and pair production with screening. Phys. Rev., 114:887, 1959. 